\title{
Mat ère

\section{Agroécologie}

Des recherches pour la transition des filières et des territoires

T. Caquet, C. Gascuel et M. Tixier-Boichard, coord.

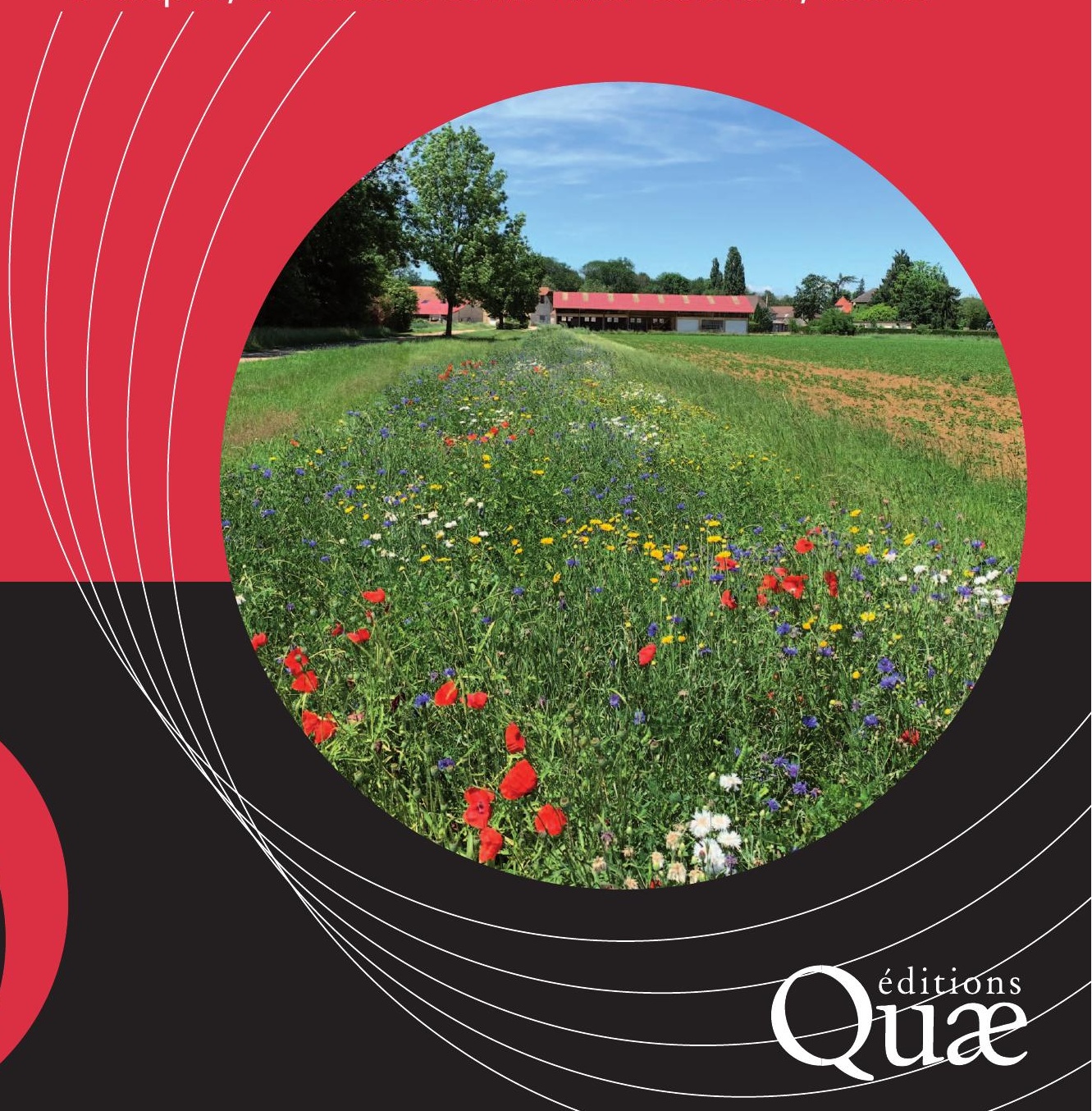





\section{L'agroécologie : \\ des recherches}

\section{pour la transition des}

\section{filières et des territoires}

Thierry Caquet, Chantal Gascuel, Michèle Tixier-Boichard, coord.

Thierry Caquet, Chantal Gascuel, Michèle Tixier-Boichard, Benoît Dedieu, Cécile Détang-Dessendre, Pierre Dupraz, Philippe Faverdin, Laurent Hazard, Philippe Hinsinger,

Françoise Lescourret, Isabelle Litrico-Chiarelli,

Françoise Médale, Hervé Monod, Sandrine Petit, Xavier Reboud, Lionel Roques, Alban Thomas, Hugo de Vries, Jean-François Soussana

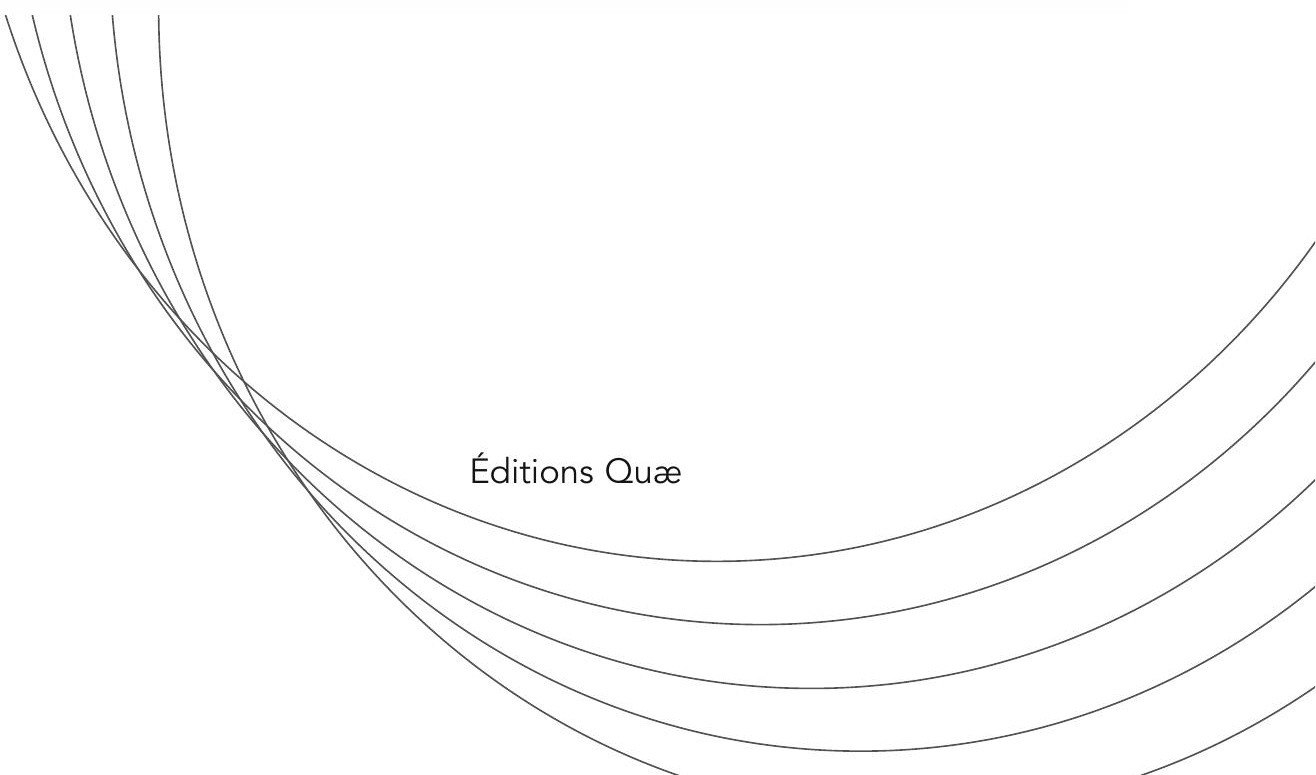




\title{
Collection Matière à débattre et décider
}

Sols artificialisés - Déterminants, impacts et leviers d'action Maylis Desrousseaux, Béatrice Béchet, Yves Le Bissonnais, Anne Ruas, Bertrand Schmitt, coord. 2019, 182 p.

Strategic management of agricultural and life science research organisations - Interface, processes and contents

Bettina Heimann, Lance O’Brien, coord. (ePub)

Impacts et services issus des élevages européens Bertrand Dumont, Pierre Dupraz, Catherine Donnars, coord. $182 \mathrm{p}$.

Peut-on se passer du cuivre en protection des cultures biologiques?

Didier Andrivon, Isabelle Savini, coord. $126 \mathrm{p}$.

\author{
Éditions Quæ \\ RD 10 \\ 78026 Versailles Cedex, France \\ www.quae.com - www.quae-open.cm \\ (C) Éditions Quæ, 2020 \\ x-ISBN (ePub) : 978-2-7592-3131-7 \\ ISSN : 2115-1229
}

ISBN : 978-2-7592-3129-4

e-ISBN (pdf) : 978-2-7592-3130-0

Le code de la propriété intellectuelle interdit la photocopie à usage collectif sans autorisation des ayants droit. Le non-respect de cette disposition met en danger l'édition, notamment scientifique, et est sanctionné pénalement. Toute reproduction même partielle du présent ouvrage est interdite sans autorisation du Centre français d'exploitation du droit de copie (CFC), 20 rue des Grands-Augustins, Paris $6^{\mathrm{e}}$. 


\section{Table des matières}

Préface

Introduction

Des principes fondateurs 10

Des attentes sociétales nationales et internationales 13

Des recherches fondées sur de nouveaux paradigmes

et de nouvelles approches 15

Références bibliographiques $\quad 17$

1. Intégrer l'agroécologie dans des systèmes agri-alimentaires

Les produits issus de l'agroécologie et leurs propriétés 20

Les stratégies des acteurs 20

L'organisation spatiale des marchés 22

La dynamique et la cohérence du système agri-alimentaire 23

Les questions de recherche 25

Références bibliographiques $\quad 29$

2. La transition agroécologique de l'exploitation agricole 31

Des avancées scientifiques récentes 32

Quelques exemples $\quad 36$

Les questions de recherche $\quad 39$

Le développement d'une nécessaire transdisciplinarité 43

Références bibliographiques $\quad 44$

3. Valoriser les processus écologiques

et hydrobiogéochimiques dans des paysages multifonctionnels

Des avancées scientifiques récentes $\quad 49$

Quelques exemples $\quad 50$

Les questions de recherche 53

$\begin{array}{ll}\text { Références bibliographiques } & 57\end{array}$ 
4. Valoriser la diversité génétique en sélection végétale et animale

Des avancées scientifiques récentes 60

Quelques exemples 62

Les questions de recherche $\quad 65$

$\begin{array}{ll}\text { Références bibliographiques } & 68\end{array}$

5. Modéliser les interactions du vivant, en lien avec les milieux et le contexte socio-économique 71

Des avancées scientifiques récentes 72

Quelques exemples $\quad 73$

Les questions de recherche $\quad 75$

Références bibliographiques $\quad 79$

6. Contribution des agroéquipements et du numérique à l'agroécologie : renforcer la prise en considération du vivant 81

Des avancées scientifiques récentes 83

Quelques exemples $\quad 86$

Les questions de recherche $\quad 88$

Références bibliographiques 92

Conclusions $\quad 95$

Diversité et diversification : observer, traduire, piloter 95

De la collecte de données biologiques en masse

à des dispositifs d'une nature nouvelle 96

Appréhender le risque et l'incertitude : modélisation

et partage d'expériences 98

Le nécessaire changement d'échelle et de niveau d'organisation 98

Perspectives 100

$\begin{array}{ll}\text { Contributeurs } & 101\end{array}$ 


\section{Préface}

Au COURS de LA DERNIÈre déCENNIE, de nombreux acteurs se sont mobilisés pour l'agroécologie à l'échelle nationale et internationale. Le ministère français en charge de l'Agriculture lance le « projet agro-écologique pour la France » en 2012. Ce projet mobilisateur pour toute l'agriculture française vise à produire autrement, en repensant les systèmes de production agricoles, en les inscrivant dans des dynamiques collectives au travers notamment des Groupements d'intérêt économique et environnemental $(\mathrm{GIEE})^{1}$. Le Conseil économique, social et environnemental (CESE) se saisit de la question de l'agroécologie et rend un avis en 2016. L'Organisation des Nations unies pour l'alimentation et l'agriculture (FAO) organise en 2014 le $1^{\text {er }}$ Symposium international sur l'agroécologie, pour la sécurité alimentaire et la nutrition, avec l'ambition de promouvoir les systèmes agroécologiques au niveau international. Elle a depuis organisé des rencontres par région du monde, dont les conclusions ont été partagées lors d'un second colloque, en avril 2018, où elle a lancé l'« Initiative de passage à l'échelle supérieure de l'agroécologie ». Dans le même temps, le Centre de coopération internationale en recherche agronomique pour le développement (Cirad) et l'Institut national de la recherche agronomique (Inra) ont mis en exergue leur convergence de vue dans une note commune ${ }^{2}$. Celle-ci a stimulé la recherche nationale et internationale, mais aussi une meilleure articulation entre les initiatives de la société et les dispositifs de recherche des pays du Nord et du Sud.

Lancé en 2011, le chantier " agroécologie » de l'Inra impulse une nouvelle dynamique de recherche. L'agroécologie est considérée comme une discipline scientifique à part entière, à l'interface entre l'écologie et l'agronomie. Ce premier chantier permet d'amplifier une vision systémique et écologique des recherches sur les agroécosystèmes, les considérant comme des écosystèmes gérés non plus aux seules fins de production agricole, mais, plus largement, de fourniture de services écosystémiques : à des visées de production de biomasse sont associés des objectifs de préservation des ressources naturelles (eau, sol, biodiversité), de patrimoines culturels (paysage), d'atténuation du changement climatique.

Cinq priorités de recherche sont alors dégagées : la connaissance et l'utilisation des interactions biologiques dans les agroécosystèmes ; l'agroécologie du paysage ; l'évaluation multicritère des agroécosystèmes, intégrant la biodiversité et la fourniture de services

1. Voir https://agriculture.gouv.fr/pres-de-10-0oo-agriculteurs-engages-dans-les-groupements-dintereteconomique-et-environnemental-giee.

2. Soussana J.-F., Côte F., 2016. Agro-écologie : le positionnement des recherches de l'Inra et du Cirad, 8 p. Voir https://www.cirad.fr/content/download/11293/132717/version/3/file/Agro-ecologie-Inra-CIRADnote-longue.pdf. 
écosystémiques ; la gestion durable des ressources en sol et en eau comme levier pour l'agroécologie ; la conception de nouveaux systèmes agricoles, mobilisant les sciences de l'homme et de la société.

En 2012, ces travaux conduisent à la production d'une synthèse ${ }^{3}$ ainsi qu'à la formulation de recommandations pour l'Inra. En 2013, un colloque est organisé par l'Inra, sous l'égide du ministère en charge de l'Agriculture, rassemblant des chercheurs, des responsables et des acteurs du monde agricole. Il constitue un moment d'échange, de mise à l'agenda de l'agroécologie pour la recherche, pour le monde agricole, et plus généralement pour l'ensemble de la société. Les interventions sont publiées dans la revue gratuite en ligne Innovations agronomiques ${ }^{4}$.

Le terme "agroécologie » diffuse dans l'Inra et dans la société comme un nouveau paradigme pour repenser les agroécosystèmes et les activités agricoles. En 2014, le séminaire "Nouveaux défis de la modélisation : l'agroécologie " ${ }^{5}$ permet de progresser dans le domaine de la représentation, de la prédiction et du pilotage des agrosystèmes. Depuis 2014, le métaprogramme EcoServ (Services rendus par les écosystèmes) met en avant une approche écosystémique des agroécosystèmes : l'agriculture est pourvoyeuse de services écosystémiques (y compris de disservices) dont on cherche à valoriser les synergies, à identifier les antagonismes, mais aussi que l'on cherche à améliorer par une vision systémique. Lancé en 2000, le programme AgriBio (Pour et sur l'agriculture biologique) est soutenu par une action spécifique de recherche en 2015. En 2019, il devient un métaprogramme de l'Inra, Métabio "Changement d'échelle de l'agriculture biologique ». L'agriculture biologique est vue comme un label fondé sur les principes de l'agroécologie. L'étude des mécanismes permettant d'amplifier les régulations biologiques et écologiques dans les agroécosystèmes est désormais à la base d'une ingénierie nouvelle dont il convient d'évaluer les performances.

En 2016, avec son document d'orientation "\#Inra2025 ", l'institut décide d'intensifier les recherches en agroécologie par l'approfondissement de certains thèmes et par l'élargissement du champ d'investigation, prenant en compte des transformations plus larges, au niveau des filières et des territoires. L'agroécologie n'est pas une simple et nouvelle façon de voir l'agronomie, mais une reconception des productions agricoles s'inscrivant dans un processus social, avec des dimensions économiques, sociologiques, alimentaires et environnementales. Cette décision se traduit en 2017 par le lancement par l'Inra d'une réflexion prospective interdisciplinaire sur la recherche nécessaire pour l'agroécologie, qui associe environ 80 chercheurs et enseignants-chercheurs.

3. Voir http://inra.dam.front.pad.brainsonic.com/ressources/afile/228001-a8d94-resource-chantier-agroecologie-fevrier-2013-4-pages.html.

4. Voir https://www6.inra.fr/ciag/Revue/Volumes-publies-en-2015/Volume-43-Mars-2015.

5. Garcia F., Gascuel-Odoux C., Soussana J.-F. (eds), 2014. Colloque sur les nouveaux défis de la modélisation : l'agroécologie, Synthèse, Inra, $49 \mathrm{p}$. 
Cet ouvrage présente le fruit de cette réflexion collective. L'objectif est de partager et de mettre en débat ces travaux, en interne et avec nos partenaires de la recherche, du monde agricole et de la société.

La création d'INRAE, fruit de la fusion de l'Inra et d'Irstea, l'élargissement des compétences, la mise en place de nouveaux métaprogrammes interdisciplinaires, de projets de territoires d'innovation vont contribuer à amplifier les recherches pour l'agroécologie, à déplacer les fronts de connaissances nécessaires, à mettre la connaissance et la coconstruction au cœur des évolutions des filières et des territoires avec l'ensemble des acteurs concernés.

Philippe Mauguin, président-directeur général d'INRAE 



\section{Introduction}

Pour faire face À l'Augmentation de la population mondiale, aux défis environnementaux et climatiques, à la raréfaction des ressources en eau et en énergies fossiles, l'adaptation, voire la rupture des modes de production agricole actuels, est devenue incontournable. Les systèmes agricoles devront considérer les services de production de biens agricoles, mais aussi d'autres services écosystémiques. Les acteurs devront pour cela être accompagnés par la recherche et la formation.

Dans les pays industrialisés de la zone tempérée, l'amélioration de la productivité de l'agriculture et de sa compétitivité économique depuis les années 1950 a été permise par un processus de modernisation qui s'est traduit par la spécialisation des systèmes de production, par l'agrandissement des exploitations et par un recours accru à des intrants de synthèse, au machinisme agricole, à des variétés végétales et à des races animales à fort potentiel productif. La spécialisation des systèmes, l'artificialisation et l'homogénéisation des milieux ont permis de faire des économies d'échelle, tant du point de vue de la production que de la collecte de produits plus standardisés, répondant mieux aux besoins des filières de transformations et des industries agroalimentaires.

Au cours de cette période, le secteur agricole s’est organisé par la création de référentiels et de structures de conseils ; les milieux naturels ont été considérés comme largement abiotiques, homogénéisés par le remembrement, le drainage ; les interactions biotiques dans le sol et dans les écosystèmes supports de l'agriculture ont été négligés. Le conseil aux exploitations agricoles a visé l'optimum de production. L'agriculture s'est industrialisée. Cette industrialisation a engendré des externalités jugées alors positives (milieux " propres », sans ravageurs, à forte productivité), mais aussi des externalités négatives (pollutions du sol, de l'eau, de l'air ; émissions de gaz à effet de serre ; perte de biodiversité) dont les conséquences sont devenues des questions cruciales depuis quelques décennies. Le constat dressé par l'IPBES ${ }^{6}$ dans son évaluation mondiale de la biodiversité et des services écosystémiques est clair : au travers du changement de l'usage des terres et de l'usage d'intrants, l'agriculture, incluant l'élevage, est l'un des principaux moteurs de l'érosion de la biodiversité (IPBES, 2019).

Un meilleur compromis entre agriculture et environnement a été recherché par une meilleure efficience des intrants, une limitation des rejets dans l'environnement, voire une démarche de reconception des systèmes agricoles. De nombreux mouvements (agriculture biologique, agriculture de conservation, agriculture raisonnée, agriculture à haute performance environnementale, éco-agriculture...) ont proposé des vocables et des concepts pour mieux conjuguer les performances économiques, sociales, environnementales et

6. Intergovernmental Science-Policy Platform on Biodiversity and Ecosystem Services (Plateforme intergouvernementale sur la biodiversité et les services écosystémiques). 
sanitaires de l'agriculture. L'agroécologie apparaît, y compris dans ce contexte des pays industrialisés, comme la voie essentielle, englobante et fondée sur des principes, pour contribuer au développement d’une agriculture durable et résiliente.

\section{Des principes fondateurs}

L'AGRó́cologie EST À LA FOIS Un dOMAINe SCIENTIFIQUe, une pratique et un mouvement social (Wezel et al., 2009), comme l'illustre la figure 1. Des définitions variées ont été proposées, qui associent à des degrés divers l'écologie à d'autres disciplines (agronomie, génétique, sociologie, etc.), à des connaissances locales ou traditionnelles, visant la durabilité des systèmes de production, voire des systèmes alimentaires, la préservation et l'utilisation de la biodiversité (Wezel et al., 2018). L'interdisciplinarité, l'interaction entre disciplines et la transdisciplinarité, l'interaction entre la recherche et la société sont, de manière partagée, centrales dans l'agroécologie.

\section{Figure 1. L'agroécologie est à la fois une pratique, un mouvement social et un domaine scientifique (d'après Wezel et al., 2009).}

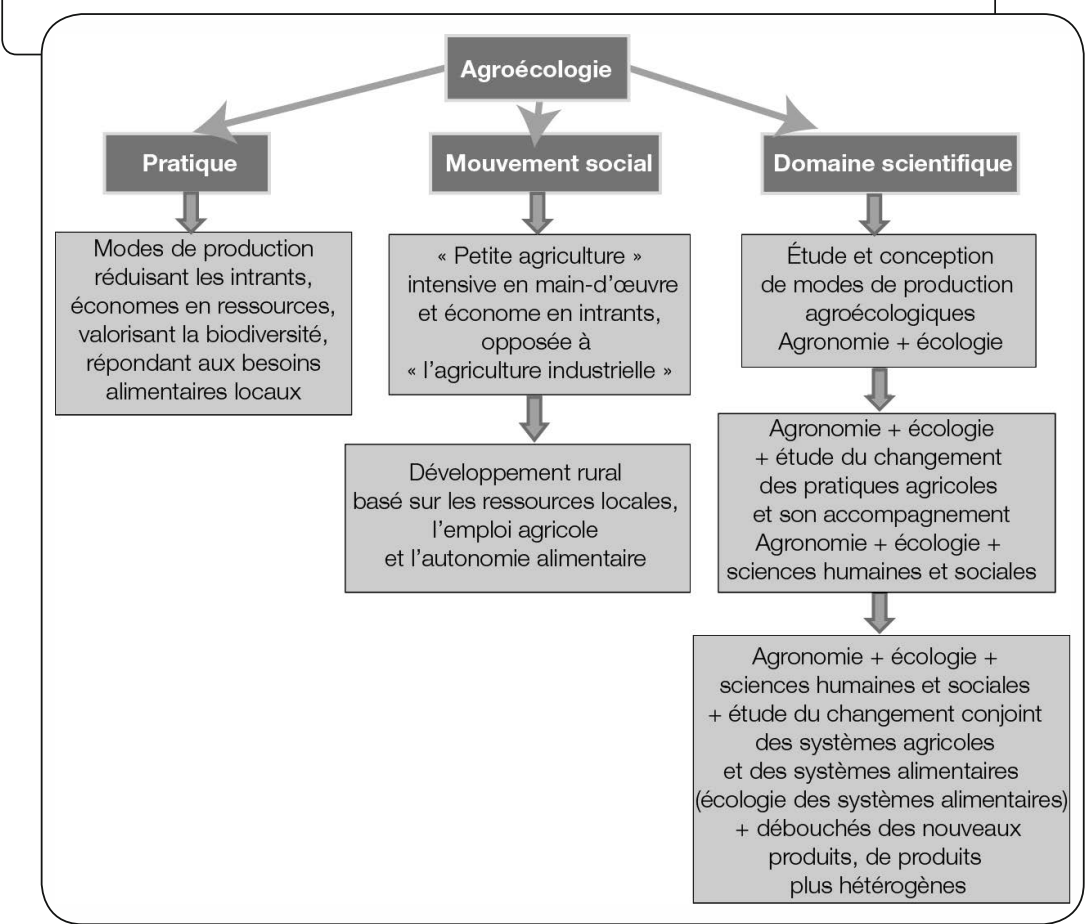




\section{Une ambition : valoriser les processus biologiques}

L'agroécologie est avant tout un nouveau paradigme qui vise à valoriser les processus biologiques pour couvrir à la fois des attentes de production agricole et d'autres services écosystémiques des agrosystèmes : protéger les ressources, contribuer à atténuer le changement climatique, préserver les habitats et les patrimoines culturels. Un corollaire est de considérer l'agroécologie comme une visée pour que, au travers des systèmes agricoles et des pratiques déployées, les agrosystèmes intègrent les fonctionnalités écologiques qui garantissent leur propre pérennité, notamment en matière de reconstitution de stocks de nutriments et de maintien du potentiel productif.

De ce premier paradigme découle un second : valoriser les processus biologiques, c'est prendre en compte plus de diversité dans les agroécosystèmes, ce qui débouche sur une plus grande diversité de produits agricoles, une plus grande hétérogénéité de chaque produit qu'il faudra transformer et intégrer dans des produits alimentaires, voire des régimes alimentaires nouveaux.

Cette définition permet de clarifier les attendus posés à la recherche. Ainsi, sous les termes de smart agriculture, ou agriculture intelligente, ou de sustainable agriculture, ou agriculture durable, se trouvent réunis des travaux plutôt d'ordre technologique sur le meilleur usage possible des ressources : cela correspond à une agroécologie dite " faible ", dans la continuité des systèmes actuels, ne revendiquant pas de saut qualitatif quant à l'efficience de l'utilisation des intrants, ni d'appel explicite à des processus biologiques en substitution de l'usage d'intrants (Duru et al., 2014). À cette agroécologie dite "faible » s'oppose une agroécologie dite "forte », définie par sa finalité de cohérence et de durabilité et par la mobilisation de processus biologiques (Duru et al., 2014). Cette agroécologie forte requiert une transformation en profondeur des systèmes de production agricole. Elle constitue l'objectif d'INRAE, parce que cette ambition ne se fera pas sans un engagement important de la recherche, parce que l'ensemble des productions agricoles, l'ensemble des disciplines académiques est concerné. Il ne faut pas y voir une volonté d'opposer, mais une ambition, celle de repenser la mobilisation des processus biologiques à tous les niveaux (espèce, race/variété, physiologie animale et végétale/alimentation et fertilité, mode d'élevage et itinéraires de culture, devenir des produits et coproduits, lien aux ressources, aux énergies, au sol et à l'eau, localisation...).

\section{Vers la reconception des systèmes de culture}

Principe de construction des systèmes agroécologiques, vouloir « valoriser les processus biologiques » nécessite le plus souvent la reconception des systèmes de culture, par exemple l'assolement, les génotypes ou les pratiques agricoles utilisées, l'articulation entre production végétale et élevage, le lien aux modes de distribution et de consommation, l'organisation des paysages, etc. Le domaine de l'agroécologie n'est pas restreint à la seule production végétale, mais, au contraire, considère les productions animales comme un pilier majeur des processus biologiques, du fait de leurs complémentarités avec les productions végétales. 
Le suivi de processus et de flux entretenus dans un équilibre dynamique de la parcelle au paysage, autorisant à la fois l'exploitation et la reconstitution des stocks organiques et minéraux et le développement de la vie dans les sols, est au cœur de cette reconception. L'agroécologie forte implique donc d'aller au-delà de la simple optimisation des systèmes agricoles.

Dans une vision très intégrative, l'agroécologie ne peut se développer sans une demande de la société, en cohérence avec les besoins de consommation alimentaire et leur organisation dans des filières et des territoires ; certains auteurs intègrent ainsi dans l'agroécologie la dimension de systèmes alimentaires (food system ; Francis et al., 2003 ; Gliessman, 2006). La reconception en profondeur des agrosystèmes, comme celle des filières et de leur organisation dans les territoires, en cohérence avec les besoins de consommation est un processus adaptatif qui se construit en avançant, dans une trajectoire qui n'est pas définie au préalable : la phase de transition apparaît donc comme un objet de recherche en tant que tel.

Cette reconception repose sur l'utilisation de principes issus de l'écologie. Une des motivations est de renforcer la résilience des agroécosystèmes, définie comme leur capacité à s'adapter aux perturbations ou à revenir à un régime d'équilibre face à un contexte changeant. Devant les incertitudes liées au changement climatique, aux évolutions sociétales ou à la volatilité des prix agricoles et alimentaires, la diversité biologique des agroécosystèmes peut constituer un facteur de résilience permettant d'atténuer les effets des perturbations. La vulnérabilité des agrosystèmes, autrefois compensée par un recours de court terme aux intrants, est désormais pensée au travers de leur résilience et d'une plus grande stabilité des productions dont la diversité biologique serait un facteur essentiel.

Figure 2. La trajectoire des systèmes agricoles : d'une phase de spécialisation à une reconception de systèmes diversifiés fondée sur les principes de l'agroécologie (d'après Tittonell, 2014).

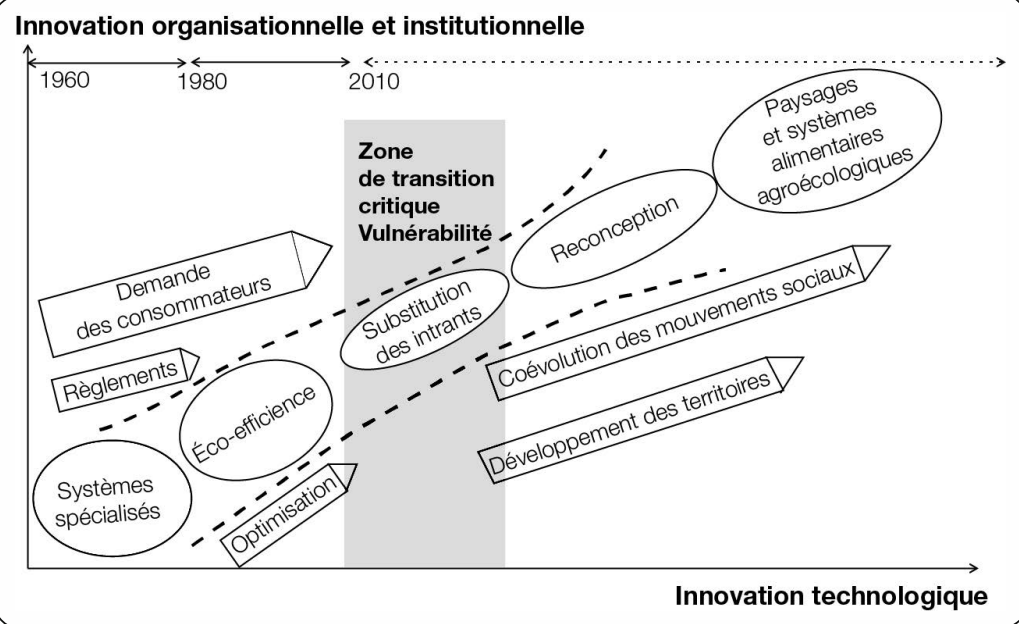


La figure 2 illustre le fait que, dans les décennies passées, les systèmes agricoles se sont spécialisés et ont été optimisés selon des principes d'éco-efficience. La transition agroécologique, qui vise la substitution des intrants par des processus biologiques, les rend temporairement plus vulnérables, mais pour aboutir à des systèmes plus divers, mieux adaptés aux milieux et aux attentes sociétales, plus résilients, fondés sur des principes d'écologie.

L'agroécologie côtoie le concept d'économie circulaire, au sens où toutes deux s'inscrivent dans le cadre du développement durable et s'inspirent notamment des notions d'économie verte, d'économie de l'usage, voire d'écologie industrielle, du bouclage des cycles en évitant au maximum le " stade déchet », limitant d'autant la consommation de matières premières et d'énergie. L'agroécologie partage avec la bioéconomie l'objectif de remplacer l'usage de ressources et de productions non renouvelables d'origine fossile par leur recyclage partiel, par la mobilisation de ressources renouvelables (photosynthèse, biologie des sols) pour leur transformation en aliments, fertilisants organiques, matériaux, bases chimiques et bioénergies variées. Si l'agroécologie partage avec l'économie circulaire et la bioéconomie l'ambition de servir une agriculture durable économe en ressources, elle s'en distingue par la place centrale qu'elle donne à la valorisation de la diversité du vivant.

\section{Des attentes sociétales nationales et internationales}

Le domAine de L'AgRó́cologie A CONNU UN ESSOR IMPORTANT depuis les années 2000, avec comme caractéristique la poursuite d'une coexistence de plusieurs visions, que ce soit en termes académiques, de méthodes de recherche ou de pratique. C'est pourquoi son périmètre et sa définition restent flous : elle n'est pas une fin en soi, mais porte des principes pour accompagner des transitions mettant les processus écologiques au cœur de la conception et de la conduite des agroécosystèmes. L'agroécologie s’inscrit dans un contexte sociétal national et international porteur. Citons-en quelques cadres de référence.

\section{Le projet agroécologique du gouvernement français}

Le projet agroécologique du ministère français en charge de l'Agriculture a été conçu, avec pour objectif d'encourager les modes de production performants à la fois sur le plan économique et environnemental, promouvant le fait d'aborder de manière articulée les différentes dimensions de l'exploitation, et au-delà celles des filières et des territoires. Il vise à produire autrement, en repensant les systèmes de production. Cela suppose un changement des pratiques agricoles, mais c'est aussi une autre façon de penser, une mutation progressive et profonde qui met l'accent sur la dimension systémique de l'activité agricole, sur des échelles larges et des pas de temps long. Il constitue désormais un cadre mobilisateur pour l'agriculture française pour repenser la formation et le 
conseil agricole. Ainsi que le premier article du code rural le souligne depuis le vote de la loi d'avenir du 13 octobre 2014, "les politiques publiques visent à promouvoir et à pérenniser les systèmes de production agroécologique, dont le mode de production biologique, qui combinent performance économique, sociale, notamment à travers un haut niveau de protection sociale, environnementale et sanitaire. Ces systèmes privilégient l'amélioration de la compétitivité des exploitations agricoles, en maintenant ou en augmentant la rentabilité économique ».

Le CESE s'est saisi de l'enjeu de l'agroécologie et a rendu un avis dont voici un extrait : «Discipline scientifique au carrefour de l'agronomie et de l'écologie, l'agroécologie peut, à travers les pratiques qu'elle promeut, contribuer à relever des défis environnementaux et socio-économiques, en transformant l'agriculture pour aller vers des systèmes alimentaires plus durables. À partir d'une analyse des freins et des leviers à son développement, le CESE a formulé un ensemble de préconisations en matière de recherche, de formation, d'adaptation des filières agroalimentaires, de réorientation des politiques publiques pour accompagner les agriculteur.rice.s dans la transition agroécologique » (Claveirole, 2016).

\section{La FAO : priorités pour l'agroécologie}

La FAO a organisé en 2014 le $1^{\text {er }}$ Symposium international sur l'agroécologie pour la sécurité alimentaire et la nutrition ${ }^{7}$, avec l'ambition de promouvoir au niveau international les systèmes agroécologiques. Il a été l'occasion de partager des expériences et de construire la base des connaissances sur l'agroécologie et a permis de parvenir à un consensus sur les priorités pour l'agroécologie. Il a surtout validé le rôle de la FAO dans la mise en œuvre et la promotion des approches agroécologiques. Ces dernières étaient déjà considérées de longue date par les agriculteurs des pays du Sud comme une alternative aux systèmes de production dominants et intensifs, car alliant une plus grande autonomie vis-à-vis des intrants, une plus forte productivité par des associations végétales explorant mieux les ressources du sol, et une moindre sensibilité aux bioagresseurs. Le symposium de 2014 a montré que l'agroécologie pouvait constituer une manière de repenser les systèmes agricoles tant dans les pays du Sud que dans les pays industrialisés. La FAO a depuis organisé des rencontres par région du monde, dont une pour la région Europe, dont les conclusions ont été partagées lors d'un second colloque, en avril 2018. L'« Initiative de passage à l'échelle supérieure de l'agroécologie » a été lancée à cette occasion ${ }^{8}$. Ce projet vise à encourager un processus de transition vers l'agroécologie qui soit plus inclusif et holistique, des systèmes agricoles aux systèmes alimentaires, par le biais d'outils de partage du savoir, notamment par la mise en place d'une plateforme des connaissances sur l'agroécologie ${ }^{9}$. Des critères visant à caractériser les systèmes agroécologiques ont été définis et un travail méthodologique pour analyser leurs performances est en cours.

7. Voir http://www.fao.org/3/a-i4327e.pdf.

8. Voir http://www.fao.org/3/l9049FR/igo49fr.pdf.

9. Voir http://www.fao.org/agroecology/fr/. 


\section{Des recherches fondées sur de nouveaux paradigmes et de nouvelles approches}

LA FIgURE 3 ILLUSTRE LES AMBITIONS ET LES CHAMPS DE RECHERCHE pour passer de systèmes conventionnels à des systèmes conduits en agroécologie.

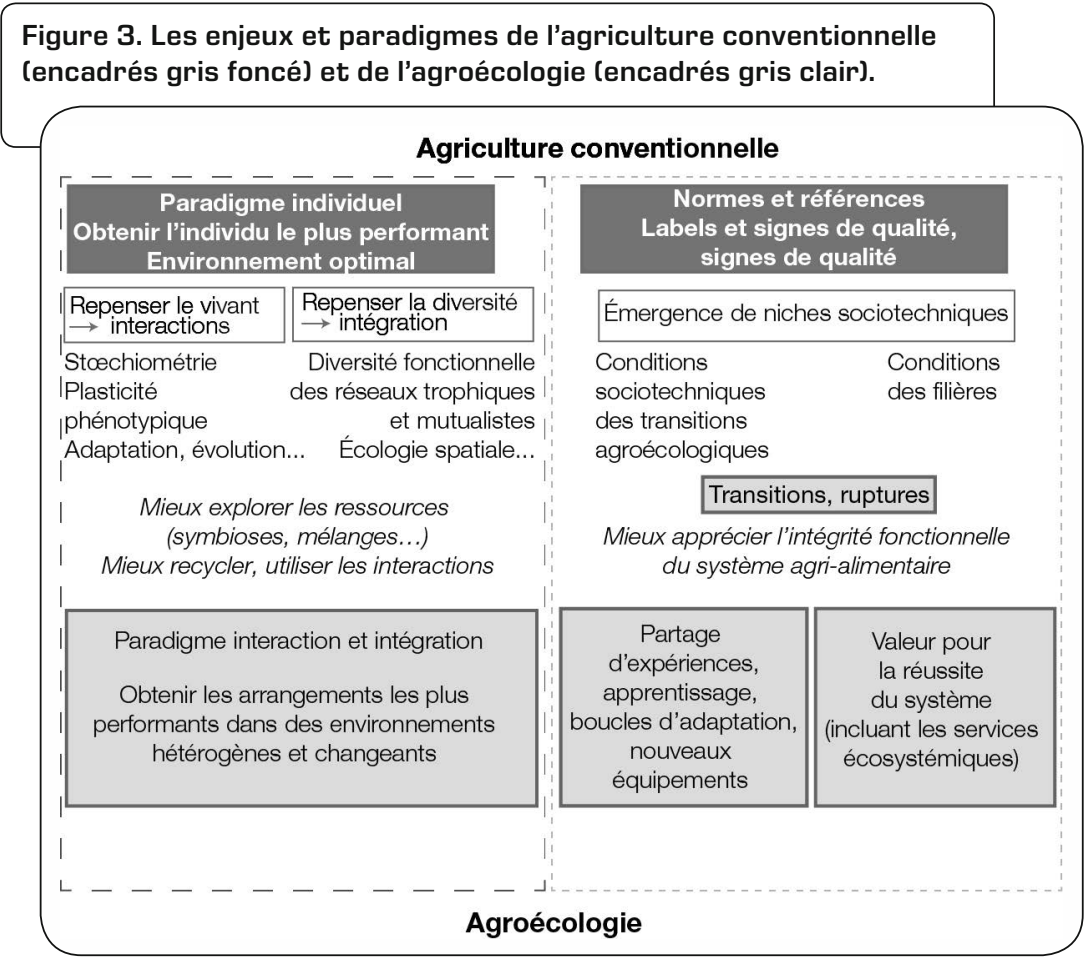

La première ambition de l'agroécologie est de changer de paradigme, de passer d'un paradigme fondé sur «l'individu idéal », qui vise à obtenir l'individu, animal ou végétal, le plus performant dans un environnement rendu optimal et qui a forgé les systèmes agricoles actuels, à un nouveau paradigme fondé sur les interactions entre individus et leur intégration dans des écosystèmes, qu'il s'agisse du champ ou du paysage. L'hypothèse sous-jacente est qu'une diversité d'individus, de variétés/races ou d'espèces sera mieux adaptée à des environnements hétérogènes et changeants du fait des interactions qu'ils entretiennent. Leurs arrangements dans le temps et l'espace pourront s'avérer aussi plus efficients, car explorant mieux les ressources en eau et en minéraux, mais surtout plus résilients aux perturbations en raison même de leur diversité. La recherche va alors 
s'intéresser aux propriétés fonctionnelles, celles qui rendent des fonctions et services écosystémiques (fourniture de biomasse, régulation du cycle de l'eau, des sols, du climat, aménités des paysages, etc.).

Ce nouveau paradigme fait appel à des notions issues de l'écologie, notamment de l'écologie fonctionnelle, qu'il s'agit d'adapter et de mettre à profit pour les agroécosystèmes : - la stœchiométrie est définie comme la proportion d’éléments chimiques, le plus souvent de carbone, d'azote et de phosphore, dans des organismes ayant des liens trophiques. Appliquée à l'écologie, la stœchiométrie étudie la propagation de ces proportions du fait d'une chaîne de réactions liées aux besoins des plantes, au fonctionnement des sols, voire au transfert au niveau des bassins versants et des écosystèmes aquatiques. Des associations végétales et animales peuvent tirer profit au mieux de ces proportions, mettre en synergie des disponibilités et des besoins variés, introduire des ressources recyclées adaptées ; - la plasticité phénotypique des espèces est une propriété qui leur permet de s'adapter à des conditions de milieux variés. Plus une espèce sera " plastique » et plus elle pourra s'adapter à des conditions variées et/ou fluctuantes. En termes de sélection génétique pour des variétés ou des races à vocation agronomique, on pourra préférer des capacités de plasticité à des optimums conçus pour des conditions standards, ce qui peut se traduire par des objectifs de sélection sur la base de variances phénotypiques ;

- les réseaux trophiques et mutualistes définissent les liens qu'entretiennent les espèces, que ce soit des liens alimentaires (trophiques) ou de simples relations à bénéfices réciproques (mutualistes), comme le sont par exemple les symbioses.

La recherche en agroécologie est donc très orientée vers l'analyse du vivant, l'adaptabilité des variétés/races et des espèces, la nature et l'importance des interactions entre individus, leurs effets d'association aux échelles supra-individuelles, pour in fine identifier les meilleurs arrangements, aider à leur pilotage et caractériser les fonctions et services écosystémiques qui en découlent. Si beaucoup de processus écologiques ont été étudiés depuis quelques années dans les agroécosystèmes, intégrer ces processus dès la phase de conception des agrosystèmes est un nouvel enjeu pour la recherche.

La seconde ambition de l'agroécologie est de passer d'un paradigme fondé sur des normes et des référentiels, qui permet leur utilisation partout et en toutes circonstances (pour le conseil, la vente...), et qui était devenu l'objectif de l'accompagnement de la production agricole ces dernières années, à un paradigme de diversification sociotechnique, spécifique d'une situation et aboutissant à des trajectoires sociotechniques, des filières, avec des transitions, voire des ruptures. Deux notions sont mobilisées : le partage d'expériences et l'apprentissage pas-à-pas, qui accompagnent les transitions et l'adaptation des systèmes à leur contexte sociotechnique ; l'identification des possibles et des risques. L'agroécologie sera davantage un chemin basé sur la mobilisation de processus et de principes qu'une norme ou un label.

Des indicateurs devront décrire ces chemins pour accompagner les consommateurs. Ces systèmes seront caractérisés par des valeurs, en lien avec la notion de services écosystémiques, des dimensions humaines, économiques et sociologiques assumées, 
reconnues, voire aidées, dans les territoires. Le plan Biodiversité dévoilé en juillet 2018 a par exemple cadré l'utilisation de produits biologiques ou locaux dans la restauration collective. Ce système de valeurs, reconnu par la société, stimule l'évolution de l'ensemble du système agri-alimentaire.

Les six chapitres qui composent cet ouvrage déclinent six enjeux.

- Intégrer l'agroécologie dans les systèmes agri-alimentaires. L'agroécologie privilégie le recours à la diversité, au recyclage des éléments, à la recherche de complémentarités, bousculant ainsi l'organisation des filières, requérant de nouvelles filières, impliquant tant les producteurs que les consommateurs ou les collectivités territoriales (circuits courts, filières sous label de qualité...).

- Favoriser la transition agroécologique de l'exploitation agricole. Cette transition est porteuse d'incertitudes pour l'agriculteur qui s'y engage. Bien la gérer implique d'en identifier les vulnérabilités, d'outiller les acteurs, en se plaçant dans une temporalité longue, en associant savoirs scientifiques et expériences d'acteurs.

- Valoriser les processus écologiques et hydrobiogéochimiques dans des paysages multifonctionnels. La dimension paysagère, incluant la distribution spatiale des éléments du paysage sur et dans le sol (« infrastructures vertes »), l'organisation spatio-temporelle des assolements et des conduites de cultures comme des élevages (« paysage des pratiques »), est une dimension essentielle de l'agroécologie.

- Valoriser la diversité génétique en sélection végétale et animale. La diversité génétique peut contribuer à la conception de systèmes agroécologiques. Les schémas de sélection pour les plantes et les animaux doivent évoluer pour améliorer la fourniture de services écosystémiques et la résilience des agroécosystèmes.

- Modéliser les interactions du vivant, en lien avec les milieux et les contextes socioéconomiques. Il s'agit de mieux outiller les chercheurs et les acteurs, en matière de représentation, de compréhension et de prédiction des dynamiques des agroécosystèmes, pour mieux identifier et gérer leurs atouts et leurs vulnérabilités.

- Identifier les besoins d'agroéquipements, les possibilités du numérique pour l'agroécologie. Ce thème interroge la contribution spécifique que peuvent apporter la technologie, les capteurs, les équipements et les services pour l'essor de l'agroécologie.

Dans chacun de ces chapitres, le contour et les enjeux sont définis ; les principales avancées scientifiques, puis des exemples de projets de recherche sont détaillés, illustrant la manière dont la recherche les a construits et les résultats auxquels ils ont conduit ; enfin, les principales priorités de la recherche pour développer l'agroécologie sont résumées.

\section{Références bibliographiques}

Claveirole C., 2016. La transition agroécologique : défis et enjeux. Les Avis du CESE, 13, 105 p.

Duru M., Farès M., Therond O., 2014. Un cadre conceptuel pour penser maintenant (et organiser demain) la transition agroécologique de l'agriculture dans les territoires. Cah. Agric., 23, 84-95. 
Francis C., Lieblein G., Gliessman S., Breland T.A., Creamer N., Harwood R., Saolomonsson L., Helenius J., Rickerl D., Salvador R., Wiedenhoeft M., Simmons S., Allen P., Altieri M., Flora C., Poincelot R., 2003. Agroecology: the ecology of food systems. J. Sustain. Agric., 22, 99-118.

Garcia F., Gascuel-Odoux C., Soussana J.F. (eds), 2014. Colloque sur les nouveaux défis de la modélisation : l'agroécologie. Synthèse, Inra, 49 p.

Gliessman S.R., 2006. Agroecology: the Ecology of Sustainable Food Systems, CRC Press, 408 p.

IPBES, 2019. Summary for policymakers of the global assessment report on biodiversity and ecosystem services of the Intergovernmental Science-Policy Platform on Biodiversity and Ecosystem Services (S. Díaz, J. Settele, E.S. Brondizio E.S., H.T. Ngo, M. Guèze, J. Agard, A. Arneth, P. Balvanera, K.A. Brauman, S.H.M. Butchart, K.M.A. Chan, L.A. Garibaldi, K. Ichii, J. Liu, S.M. Subramanian, G.F. Midgley, P. Miloslavich, Z. Molnár, D. Obura, A. Pfaff, S. Polasky, A. Purvis, J. Razzaque, B. Reyers, R. Roy Chowdhury, Y.J. Shin, I.J. Visseren-Hamakers, K.J. Willis, C.N. Zayas, eds), IPBES Secretariat, Bonn, Germany.

Soussana J.-F., Côte F., 2016. Agro-écologie : le positionnement des recherches de l'Inra et du Cirad, 8 p.

Tittonell P., 2014. Ecological intensification of agriculture - sustainable by nature. Curr. Opin. Environ. Sustain., 8, 53-61.

Wezel A., Bellon S., Doré T., Francis C., Vallod D., 2009. Agroecology as a science, a movement and a practice. A review. Agron. Sust. Dev., 29, 503-515.

Wezel A., Goris M., Bruil J., Félix G.F., Peeters A., Bàrberi P., Bellon S., Migliorini P., 2018. Challenges and action points to amplify agroecology in Europe. Sustainability, 10, 1598. 


\section{Intégrer l’agroécologie dans des systèmes agri-alimentaires}

L'AGRoÉCOLOGIE PRIVILÉGIE LE RECOURS À LA DIVERSITÉ, qu'elle soit génétique (association de variétés, recherche de rusticité...), spécifique (association de cultures, diversité d'espèces) ou fonctionnelle (agroforesterie, association culture-élevage...). Elle s'appuie sur le recyclage des ressources et la recherche de complémentarités entre productions. La mise en œuvre de ces principes de l'agroécologie entraîne une plus grande diversité des produits agricoles et une plus grande hétérogénéité de chacun d'eux, alors destinés à un plus grand nombre de secteurs, de consommateurs et d'utilisateurs.

Quelles sont les conséquences de l'augmentation de la diversité des produits et de l'hétérogénéité de chacun d'eux, tout au long de la chaîne de production et de transformation, de mise en marché et de consommation ? Bien appréhender les changements de l'ensemble du système vers un monde plus divers, plus hétérogène, est essentiel. La notion de filière ${ }^{10}$ est alors sans doute trop restrictive lorsqu'elle désigne l'ensemble des activités complémentaires qui concourent, de l'amont à l'aval, à la réalisation d'un produit fini, et on parlera de système agri-alimentaire. Ce qui nous intéresse en effet ici vise à relier l'ensemble des acteurs, et à envisager le produit finalement consommé dans toutes ses dimensions : - mettre en valeur l'hétérogénéité du produit, et non plus la gommer ou l'homogénéiser ; - considérer le système agriculture-environnement-alimentation, et prendre en compte l'ensemble des acteurs de ce système et leurs interactions (producteurs, fournisseurs, transformateurs, consommateurs, associations citoyennes, politiques publiques) ;

- approcher les transitions en distinguant les actions qui visent l'efficacité, la substitution et/ou la reconception selon le modèle ESR ${ }^{11}$ de Hill (1985).

Le déploiement de l'agroécologie doit questionner le système agri-alimentaire sous différents prismes que le cadre de la théorie des jeux permet de préciser :

- les produits issus de l'agroécologie, comme pièces du jeu :

- qu'est-ce qu'un produit issu de l'agroécologie?

- quels sont les liens entre les ressources et les produits, en particulier sous l'angle de la diversification et de l'hétérogénéité ?

- les stratégies des acteurs, comme joueurs :

- les pratiques et les préférences des consommateurs, ce qu'elles sont et comment les faire évoluer,

10. Voir https://www.insee.fr/fr/metadonnees/definition/c1734.

11. ESR : efficacité, substitution, reconception. 
- l'organisation des marchés, leur évolution et l'importance des normes et des standards,

- l'action publique, et en particulier la Politique agricole commune (PAC), mais plus largement l'articulation entre politique agricole, politique environnementale et politique de santé pour faire évoluer le système ;

- l'organisation spatiale des marchés et les conditions exogènes, comme terrain de jeu, en tenant compte du commerce international, des contraintes et des opportunités territoriales ;

- la dynamique du système, en tenant compte de l'ensemble des acteurs et de leurs démarches, les ressources et les produits réels et potentiels, les interactions entre sous-systèmes (territorialisés ou autres), les impacts (positifs et/ou négatifs) pour adapter les stratégies, les risques et les bénéfices de ces stratégies.

\section{Les produits issus de l'agroécologie et leurs propriétés}

CE THÈME EST TRÈS VASTE ET LES TRAVAUX MEnÉS jusqu'à présent concernent essentiellement l'analyse des propriétés des produits issus de l'agriculture biologique et de la diversité des formes d'agriculture (Abecassis et al., 2018). Les effets des modes de production agroécologique sur les propriétés des produits transformés n'ont été analysés en profondeur ni sur leurs qualités spécifiques, ni sur leurs potentiels de marché existant et/ou innovant. Toutes les étapes de transformation intervenant dans la définition des propriétés des produits doivent être analysées : les procédés, les modalités de stabilisation des propriétés (emballage, stockage), depuis la distribution de produits hétérogènes jusqu'aux préparations finales (cuisinées, prêt-à-consommer...) et au recyclage.

\section{Les stratégies des acteurs}

\section{Les pratiques des consommateurs}

Un lien peut être établi entre les propriétés des produits, les pratiques des consommateurs, leur propension à payer et les instruments qui favorisent le développement de la consommation de ces produits. Dans une méta-analyse, Dolgopolova et Teuber (2018) ont mis en évidence des consentements à payer plus élevés et assez homogènes pour des produits alimentaires ayant des attributs du registre « bon pour la santé ». Sörqvist et al. (2013) ont montré que les produits "verts » ou encore écolabellisés étaient perçus comme ayant de meilleures qualités organoleptiques. L'aversion au risque, l'aversion à la perte et l'aversion à l'inégalité (les individus seraient altruistes envers les moins favorisés et envieux à l'égard des plus favorisés) font partie des motivations des consommateurs pour adhérer à un contrat d'agriculture locale (Bougherara et al., 2017). 
Si certaines formes de diversification présentes dans les contrats d'agriculture locale sont valorisées, ce n'est pas toujours le cas.

L'étiquetage constitue un signal important pour l'adoption par les consommateurs, comme l'ont montré les travaux sur l'agriculture biologique (Asioli et al., 2017 ; Drexler et al., 2018), et l'étiquetage environnemental, initié dans le cadre du Grenelle de l'environnement, s'inscrit dans cette dynamique. Enfin, l'analyse de la perception des consommateurs vis-à-vis des produits non alimentaires comme les biomatériaux, les biomolécules pour la pharmacie, la cosmétique, la chimie verte, etc., jusqu'à la bioénergie produite et utilisée localement (méthanisation, villes autonomes en énergie...) est également essentielle (Sijtsema et al., 2016).

\section{L'organisation des marchés : importance des normes et des standards}

La mise en place de normes (ensemble de règles légiféré par un organisme de normalisation) et de standards (ensemble de recommandations préconisées par un groupe d'utilisateurs) doit favoriser et organiser les relations entre les différents acteurs des marchés.

La certification et les cahiers des charges contribuent à établir la confiance entre les acteurs, sans pour autant supprimer l'asymétrie d'information, lorsque les pratiques aboutissent à des attributs difficilement vérifiables avant et après l'achat (attributs de croyance) tels que : moins de résidus d'intrants, plus de fibres, plus de vitamines, meilleure texture ou meilleur goût. Une manière de réduire l'asymétrie d'information entre le producteur et le consommateur est d'utiliser la labellisation (Bonroy et Constantatos, 2015). Une autre dimension à considérer est celle de l'organisation des filières, et notamment leur longueur. Les contrats d'agriculture locale comme les Amap (Associations pour le maintien d'une agriculture paysanne) valorisent certains types de production et constituent des outils de partage du risque entre producteurs et consommateurs (Sproul et al., 2015). Ainsi le projet de recherche Ici.C.Local ${ }^{12}$ analyse l'organisation des circuits courts jusqu'à la mise en place d'une marque, déposée en 2014 à l'INPI (Institut national de la propriété industrielle). Cette démarche innovante et simple d'utilisation consiste à signaler les produits en circuits courts dans les lieux de vente au détail. Elle peut être utilisée par tous les acteurs économiques des circuits courts : commerçants, artisans, agriculteurs, producteurs et transformateurs.

Les questions juridiques sont aussi importantes pour comprendre les freins à la mise en marché de produits, en particulier dans le cadre du règlement sur les nouveaux aliments de l'Union européenne et des lois concernant les négociations de prix (les règles de concurrence, etc.). En effet, en vertu de la réglementation européenne, la mise en marché des aliments n'ayant pas été consommés de façon significative avant mai 1997 demande le montage et l'instruction d'un dossier complexe. De même, les règles garantissant la libre concurrence au sein de l'Union européenne peuvent impacter le positionnement des produits sur le marché.

12. Voir http://www.sad.inra.fr/Partenariat-innovation/Ici.C.Local-Valoriser-les-circuits-courts-dans-lesterritoires/Ici.C.Local-une-demarche-innovante-protegee-par-une-marque/(key)/o ; contact Yuna Chiffoleau. 


\section{Les politiques publiques}

Si les questions de certification relèvent de l'action publique de façon générale (et pas uniquement des politiques publiques), l'introduction de nouveaux modes de production dans les filières peut être soutenue par des instruments de politiques publiques, notamment dans le cadre du « verdissement » de la PAC et des politiques environnementales (la directive Eau par exemple).

Des travaux sur les paiements pour services environnementaux (PSE) viennent alimenter le débat (Tacconi, 2012 ; Duval et al., 2016 ; Etrillard, 2015). Il s'agira de :

- distinguer les services fournis à l'agriculteur des services fournis à la société pour lesquels une rémunération peut être justifiée ;

- définir les conditions et les niveaux de rémunération au-delà du coût d'opportunité de l'action mise en œuvre ;

- mettre en œuvre la façon dont les différentes formes de rémunération peuvent être appliquées, via un soutien public et/ou via le marché.

Enfin, les travaux d'Agrimonde-Terra ont montré tout l'intérêt d'une réflexion sur les enjeux de sécurité et de souveraineté alimentaire dans un contexte où les pratiques agroécologiques deviendraient majoritaires. L'impact sur la situation des pays du Sud mérite une attention particulière.

\section{L'organisation spatiale des marchés}

\section{Les dimensions territoriales et internationales}

L'organisation des marchés à différentes échelles a été abordée par le prisme de deux thématiques :

- les mécanismes de localisation des différents maillons de la chaîne (production, transformation, consommation), les formes de concentration et de dispersion selon les contraintes d'accès aux marchés, mais aussi les contraintes environnementales ;

- les conséquences des décisions européennes du développement des pratiques, notamment agroécologiques, sur les pays tiers (Boysen et al., 2016).

Plus largement, les contextes de production sont à prendre en compte, en particulier les caractéristiques géographiques, culturelles, sociales, historiques, pour expliquer la disparité des freins ou des leviers selon ces conditions locales.

\section{Exemple du programme Rebecca :}

\section{Recherche biomasse-énergie canne à Capesterre (Guadeloupe)}

Afin de contribuer aux objectifs ambitieux de la Guadeloupe en matière de transition énergétique, les chercheurs d'INRAE, du Cirad et le partenaire industriel Quadran ont mené 
un travail prospectif autour d'un projet portant sur le dimensionnement d'une filière biomasse-énergie durable et d'une unité pilote de production électrique en Guadeloupe, et sur l'identification des conditions d'émergence d'une filière agro-industrielle de production d'électricité à partir de biomasse issue de variétés de canne fibreuses cultivées localement. Afin d'éclairer les choix des décideurs de la politique régionale, l'étude a fourni des réponses sur les aspects suivants : modalités de culture, conditions de rémunération et accompagnement des agriculteurs, dimension et localisation de la centrale électrique, bilan environnemental. Fondé sur des scénarios territorialisés de déploiement de systèmes de culture adaptés aux conditions climatiques, intégrant tous types d'exploitations et de problématiques territoriales (énergie, eau, déchet), le projet a permis de répondre aux attentes d'une grande diversité d'acteurs et de filières. Un projet de création de centrale électrique de $12 \mathrm{MW}$ est actuellement porté par l'industriel partenaire du projet.

\section{La d)ynamique et la cohérence du système agri-alimentaire}

L'ANALYSE De LA dYNAmique du SYStème doit tenir compte entre autres de l'ensemble des acteurs et de leurs démarches, des ressources et des produits réels et potentiels, des interactions entre sous-systèmes territorialisés, des impacts (positifs et/ou négatifs) pour adapter les stratégies, des risques et bénéfices des diverses stratégies. La compréhension des relations entre les acteurs du système constitue le dernier angle d'analyse important, en particulier la connaissance des sources de la valeur (économique, environnementale, sociale) et des pouvoirs de marché, leur évaluation et la compréhension de leur répartition. Des analyses multicritères de la durabilité des systèmes en agriculture biologique proposent des éléments de réponse.

\section{La valorisation de la diversité et de l'hétérogénéité des ressources issues de l'agriculture}

Cette valorisation est un sujet clé, car une grande partie des coproduits et des sousproduits n'est pas valorisée ou pourrait l'être mieux (Pöyry, 2011). Ce sujet est très présent dans diverses conférences telles que «Bioraffinerie pour l'alimentation, l'énergie et les matériaux $»^{13}$.

Les volumes importants de coproduits issus des grandes cultures constituent une piste intéressante de valorisation en raison de leur potentiel économique en quantité et en prix, les rendant compétitifs vis-à-vis des produits issus de la filière pétrolière. De nombreux exemples illustrent ces possibilités :

- des procédés de valorisation des protéines végétales à grande échelle par l'institut mutualisé Improve ${ }^{14}$;

13. Voir http://www.bffm2015-congress.eu/.

14. Voir http://www.improve-innov.com/. 
- la production de bioénergie de deuxième et troisième générations par le projet Futurol ${ }^{15}$; - des unités de méthanisation sous différentes formes installées dans les champs (Charnier et al., 2017);

- la production innovante et la valorisation intégrale de Miscanthus, en créant des chaînes de valeur nouvelles ${ }^{16}$.

Les coproduits issus de la production agroécologique seront probablement en quantité plus réduite, plus diverse et hétérogène. L'enjeu sera de capter la valeur qui pourra être incorporée dans des produits de niche, avec des fonctionnalités spécifiques. Pour cela, le développement de technologies à des petites échelles compétitives sera crucial, par exemple la bioraffinerie directement réalisée au champ (de Vries et al., 2018). Des modèles d'affaires et des concepts logistiques seront à adapter. La saisonnalité des productions et les caractéristiques des territoires sont à prendre en compte. Des exemples prometteurs d'une bonne valorisation de produits issus d'une production locale pourraient donner des impulsions déterminantes : - le projet FUI Green Epoxy propose de valoriser les tannins pour des produits issus de l'agroécologie ;

- les projets méditerranéens ArimNet2 et Pyrodigest valorisent l'amendement agricole, le biochar ;

- le projet européen, intégratif et holistique NoAW (No Agricultural Waste ${ }^{17}$ ) présente des exemples de méthanisation, de production de polyesters biodégradables, de biofertilisants et une typologie de business models circulaires ;

- Organic’Vallée (une approche d'économie circulaire locale), Grap'Sud (produits innovants issus des coproduits viticoles), Bâtir en balles (biomatériaux de construction à partir de paille de riz) et AgriCarbone sont des projets intermédiaires entre les producteurs des coproduits et les utilisateurs.

\section{Agroécologie et produits traditionnels}

En Europe, les grands projets centrés sur les produits alimentaires traditionnels ont été initiés dans les années 2000. Le premier projet intégratif européen a été «Traditional foods: from culture, ecology and diversity, to human health and potentials for exploitation » $\left(\right.$ TrueFood $\left.^{18}\right)$. Ce projet a montré un large spectre d'innovations possibles, comme des produits avec des propriétés nutritionnelles améliorées : des fromages locaux avec un taux de peptides bioactifs élevé, des jambons crus et secs avec un taux de sel substantiellement réduit. D'autres innovations concernent des protocoles de surveillance de pathogènes potentiels et des emballages actifs et intelligents pour des produits frais, les premiers permettant d'augmenter la durée de conservation et les seconds une surveillance de la qualité des produits (Cotillon et al., 2013).

15. Voir https://www.projetfuturol.com/IFP_a26.html.

16. Voir https://www.grace-bbi.eu/.

17. Voir https://noaw2020.eu/.

18. Voir Rossi, 2012 ; http://www.basefood-fp7.eu/www.basefood-fp7.eu/content/download/5952/ 55880/file/rossi.pdf. 
Le projet TrueFood a été prolongé par des projets européens tels que Trade-It ${ }^{19}$ (Traditional Foods, Entrepreneurship, Innovation and Technology Transfer) ou Trafoon ${ }^{20}$ (Traditional Food Network to Improve the Transfer of Knowledge for Innovation). Ce projet a porté sur des artisans traditionnels dans les secteurs du lait, de la viande et de la boulangerie, avec comme objectifs le partage des expériences pratiques au niveau européen et le transfert des connaissances vers des petites et moyennes entreprises (PME) et des micro-entreprises, y compris des entreprises familiales.

Au sein de l'Union européenne, les PME du secteur agroalimentaire subissent la pression croissante de l'ouverture des marchés, de la demande accrue des consommateurs pour des produits alimentaires standardisés à des prix compétitifs, dans un contexte de croissance importante des grands distributeurs et la nécessité de se conformer aux réglementations nationales et européennes. Le projet Trafoon a lancé un réseau de transfert de connaissances dès 2013 afin de soutenir les PME d'aliments traditionnels, et plus particulièrement les produits alimentaires - bio et locaux - à base de céréales, poissons, fruits, olives, légumes et champignons ${ }^{21}$. Ce réseau met en contact des chercheurs, des experts dans le transfert de connaissances et des associations de PME de 14 pays européens afin de favoriser le transfert durable de l'innovation et de l'entrepreneuriat dans le secteur agroalimentaire au bénéfice des régions européennes et de leurs consommateurs. Ce projet a fortement stimulé l'interaction entre les PME d'aliments traditionnels, les associations de PME et les instituts de recherche afin d'augmenter le transfert de connaissances vers les PME, mais aussi d'identifier et de mener à bien les recherches utiles aux PME agroalimentaires. À travers ce projet, les besoins des PME dans toute l'Europe ont été analysés et les innovations disponibles les plus récentes ont fait l'objet de démonstrations et de journées de formation adaptées, par exemple dans le domaine du blé dur à Montpellier (Mandato et al., 2018).

\section{Les questions de recherche}

\section{L’analyse des propriétés des produits issus de l'agroécologie}

L'analyse des propriétés des produits issus de l'agroécologie cherche à répondre aux questions suivantes :

- au cœur des systèmes agri-alimentaires, des produits pourraient-ils être identifiés comme « agroécologiques »?

- quels sont les impacts des pratiques sur les produits ?

- quelles sont les conséquences pour les phases de transformation?

19. Voir https://cordis.europa.eu/project/rcn/110709/factsheet/en.

20. Voir https://www.trafoon.eu.

21. Voir https://www.trafoon.eu/information-shop/. 
Ces interrogations se déclinent en une série de questions plus précises :

- de quoi parle-t-on quand on parle de produits issus de l'agroécologie ? Quels sont les impacts des pratiques agroécologiques sur les produits, notamment sur les dimensions nutritionnelles ? Quels sont les indicateurs pour les qualifier?

- quelles sont les propriétés des différents produits, à la phase de production puis de transformation, et pour quel degré de transformation (non transformé, différents niveaux de formulation, stocké, emballé) ?

- quelles sont les approches technologiques nécessaires pour ces produits ?

- faut-il mélanger ces produits pour les homogénéiser ou, au contraire, les spécialiser pour de nouvelles sources de valeurs (caractéristiques des produits, contexte de production, etc.) ?

- comment prendre en compte les coproduits pour une utilisation optimale des ressources renouvelables, dans une vision intégrative de bioéconomie?

Ce thème autour de la qualification des produits fondés sur les principes de l'agroécologie et autour des enjeux de transformation associés à ces produits est très vaste. Il mène aux questions des coproduits et des relations entre agroécologie et bioéconomie, incluant l'économie circulaire, en particulier la réutilisation des produits partiellement consommés ou utilisés. Certaines de ces questions sont déjà abordées dans le contexte de l'agriculture biologique et, plus largement, dans celui de la diversité des formes d'agriculture.

\section{L'analyse des pratiques des consommateurs et de leurs évolutions}

Dans la mesure où les conséquences des pratiques agroécologiques sur les produits seraient précisées, des liens peuvent être faits entre les produits qui en sont issus et les pratiques des consommateurs :

- certaines caractéristiques des produits liées à l'agroécologie (environnementales, production locale ou non locale, conditionnement) font-elles l'objet d'un consentement à payer et sur quelles bases ? Quelles sont les motivations des consommateurs à adhérer à un contrat d'agriculture locale " agroécologique » ? Quel est le rôle des consommateurs dans le développement des approches agroécologiques?

- sur quels fondements est-il important de communiquer avec les consommateurs pour encourager l'adoption de ces produits ? Quel type d'étiquetage doit-on développer (voir les travaux sur l'étiquetage nutritionnel ${ }^{22}$ ) ?

- comment positionner le produit (un standard minimum, une marque...) pour intégrer une augmentation de la diversité de l'offre et une préférence pour des produits plus hétérogènes?

- quelle serait la perception des consommateurs vis-à-vis des produits non alimentaires issus de l'agroécologie (biomatériaux pour la construction, les fibres naturelles, la cosmétique...)?

22. Voir http://alimentation-sante.org/wp-content/uploads/2017/o3/Rapport-CS-définitif-14-mars.pdf. 
Les questions soulevées concernent entre autres les labels à développer, les modalités de communication ou de formation pour sensibiliser les consommateurs ${ }^{23}$.

Des travaux menés sur les produits de l'agriculture biologique, commercialisés sous la marque Bleu-Blanc-Cœur ou encore dans différents contextes (produits locaux, avec un contenu « environnemental » ou « bon pour la santé »), apportent des éléments de réponse et des pistes pour des travaux futurs. Des liens peuvent aussi être faits avec les travaux sur l'étiquetage nutritionnel, sur les impacts environnementaux (approche par l'analyse du cycle de vie, base de données Agribalyse ${ }^{24}$ ). Les réflexions engagées dans les projets de Territoires d'Innovation, comme le projet Agroécologie-Dijon, le projet Terres de Sources du Bassin rennais, ou le projet Ouesterel sur le bien-être animal, et leurs questionnements sur l'étiquetage et le consentement à payer des consommateurs pourraient aussi être mobilisés.

\section{L’organisation des marchés : l'importance des normes et des standards}

Sur ces thèmes, les études menées sur l'agriculture biologique sont également très utiles : la certification en agriculture biologique est en pratique un outil de valorisation. Cependant, l'agroécologie n'est pas une « dégradation de l'agriculture biologique », c'est une trajectoire. Il est donc important de travailler précisément sur l'intérêt d'une certification et de normes agroécologiques, sur la coévolution des différents modèles en prenant en compte toutes les activités, de la production jusqu'à la consommation et à l'utilisation des produits finaux. C'est une des pistes que va d'ailleurs privilégier le métaprogramme Métabio d'INRAE, qui porte sur le changement d'échelle de l'agriculture biologique.

\section{Les politiques publiques}

Les enjeux sur le « verdissement » de la PAC font l'objet d'un intérêt particulier du groupe de travail d'INRAE dédié au devenir du dispositif européen et de sa déclinaison française. Les pratiques agroécologiques y tiennent une place centrale, tant via les mesures de conditionnalité que via les mesures agro-environnementales et climatiques. Des travaux doivent se poursuivre, notamment sur les formes que peuvent prendre les paiements pour services environnementaux, un outil qui pourra favoriser la mise en œuvre de pratiques agroécologiques.

Les travaux sur les liens entre la politique agricole et les enjeux alimentaires et de santé, d’une part, et entre la politique agricole et les outils qui lui sont consacrés, d'autre part, demandent à être renforcés. Il sera intéressant de prendre en compte les conséquences

23. Voir par exemple https://www.hohenlohe.de/Typisch/Naturparadies-Hohenlohe/Gruener-Sueden/ Bioenergiedoerfer-in-Hohenlohe.html.

24. Voir https://www.ademe.fr/expertises/produire-autrement/production-agricole/passer-a-laction/dossier/levaluation-environnementale-agriculture/loutil-agribalyser. 
des approches de l'économie circulaire pour réutiliser intelligemment les ressources localement ou plus globalement. Les dimensions spatiales et temporelles des flux de matière méritent réflexion pour comprendre les avantages et les désavantages de l'utilisation de produits ou de coproduits issus de l'agroécologie.

\section{Les dimensions territoriale et internationale de l'organisation des marchés}

Les principales questions portent sur les impacts environnementaux de la localisation des activités.

Quels sont les effets de l'essor de pratiques agroécologiques ou d'approches localisées d'économie circulaire ? Cela peut-il conduire à une réorganisation des filières, des marchés? On retrouve ici indirectement l'importance d'une réflexion systémique qui rejoint celle de la bioéconomie.

Quelles sont les implications potentielles sur les autres pays, via les marchés, des options françaises sur le développement de l'agroécologie ? Il faut aborder notamment la notion de fuite, mécanisme selon lequel la délocalisation de productions fortement émettrices de gaz à effet de serre vers des pays dotés d'une législation moins contraignante conduit in fine à une augmentation de leurs émissions, mécanisme bien connu dans la littérature consacrée au changement climatique.

Enfin, quelles sont les implications des multi-usages des produits et des coproduits pour des marchés divers ? L'idée sous-jacente est d'expliciter les interactions entre l'organisation des activités à l'échelle régionale et à l'échelle internationale.

\section{La cohérence du système}

Les analyses multicritères permettent d'étudier la durabilité des systèmes selon différentes dimensions (économique, environnementale, sociale...) et d'expliciter la cohérence du système. De manière complémentaire, il est nécessaire d'étudier comment vont s'articuler les systèmes agroécologiques, avec d'un côté les systèmes conventionnels qui eux-mêmes évolueront, et de l'autre les systèmes en agriculture biologique. Les questions à aborder concernent tant la répartition des efforts respectifs que l'organisation de leurs interrelations. Ainsi, sur quel(s) acteur(s) porte l'effort de la réduction des pressions sur le milieu ? L'agriculteur ne devant pas supporter seul cet effort, les acteurs, dans une approche circulaire, sont tous concernés et parties prenantes pour trouver des solutions pertinentes. Alors les formes d'organisation en amont et en aval de la production doivent être analysées pour comprendre leur impact sur le déploiement des pratiques agroécologiques. Il sera ainsi possible d'étudier le verrouillage des systèmes par l'amont, notamment dans le cas des filières avec un degré élevé d'intégration. Enfin, comment imaginer des systèmes mixtes comprenant des produits agricoles issus de pratiques agroécologiques et de pratiques conventionnelles, et de quelle manière ces systèmes peuvent-ils évoluer vers l'agroécologie? 
L'ensemble de ces questions appelle des travaux méthodologiques, principalement sur trois points.

1. L'acquisition, la gestion et l'analyse des données sont nécessaires à toutes les échelles : l'échelle micro (individu, produit, entreprise) et l'échelle macro (régions, pays, grandes régions du monde). La construction de référentiels nécessite, d'une part, un travail méthodologique sur les indicateurs à suivre pour qualifier des pratiques agroécologiques et les produits qui en sont issus et, d'autre part, la construction et le suivi de bases d'informations tant sur la qualité des produits que sur la consommation des ménages.

2. La modélisation, avec plusieurs grandes familles de modélisation des systèmes agrialimentaires, a pour but d'évaluer l'intégration de pratiques agroécologiques : l'analyse du cycle de vie et les analyses multicritères pour l'évaluation de leur durabilité selon différentes dimensions ; les modèles mondiaux pour comprendre les répercussions de changements locaux à l'échelle de grandes régions (par exemple, GlobAgri AgrimondeTerra ; Le Mouël et al., 2018) ; les approches de type systèmes complexes et la mobilisation de la théorie des jeux.

3. Les études de cas seront primordiales pour bien comprendre la diversité des systèmes, leurs contraintes, l'hétérogénéité des ressources, des produits, des acteurs, des marchés, etc. La mise en place et la gestion de plateformes et de centres d'expérimentation serviront de bases de démonstration et de promotion des activités participatives avec des acteurs divers. Les dispositifs expérimentaux d'INRAE peuvent également illustrer des cas, des options, ou tester des idées créatives avec des acteurs divers (citoyens, consommateurs, utilisateurs, etc.).

\section{Références bibliographiques}

Abecassis J., Cuq B., Escudier J.-L., Garric G., Kondjoyan A., Planchot V., Salmon J.-M., de Vries H., 2018. Food chains: the cradle for scientific ideas and the target for technological innovations. Innov. Food Sci. Emerg. Technol., 46, 7-17.

Asioli D., Aschemann-Witzel J., Caputo V., Vecchio R., Annunziata A., Næs T., Varela P., 2017. Making sense of the "clean label" trends: a review of consumer food choice behavior and discussion of industry implications. Food Res. Int., 99, 58-71.

Bonroy O., Constantatos C., 2015. On the economics of labels: how their introduction affects the functioning of markets and the welfare of all participants. Am. J. Agricult. Econ., 97, 239-259.

Bougherara D., Gassmann X., Piet L., Reynaud A., 2017. Structural estimation of farmers' risk and ambiguity preferences: a field experiment. Eur. Rev. Agric. Econ., 44, 782-808.

Boysen O., Jensen H.G., Matthew A., 2016. Impact of EU agricultural policy on developing countries: a Uganda case study. J. Int. Trade Econ. Dev., 25, 377-402.

Charnier C., Ramso R., Latrille E., Steyer J.-P., Miroux J., 2017. Sécurisation des performances et optimisation de l'alimentation des unités de méthanisation. Journées Recherche et Industrie biogaz méthanisation - JRI 2017, Beauvais, 11-13 avril 2017. 
Cotillon C., Guyot A.C., Rossi D., Notarfonso M., 2013. Traditional food: a better compatibility with industry requirements. J. Sci. Food Agric., 93, 3426-3432.

Dolgopolova I., Teuber R., 2018. Consumers' willingness to pay for health benefits in food products: a meta-analysis. Appl. Econ. Persp. Pol., 2, 333-352.

Drexler D., Fiala J., Havlíčková A., Potůčková A., Souček M., 2018. The effect of organic food labels on consumer attention. J. Food Prod. Market., 24, 441-455.

Duval L., Binet T., Dupraz P., Leplay S., Etrillard C., Pech M., Deniel E., Laustriat M., 2016. Paiements pour services environnementaux et méthodes d'évaluation économique. Enseignements pour les mesures agro-environnementales de la Politique agricole commune. Rapport SSP, OréadeBrèche, $135 \mathrm{p}$.

Etrillard C., 2015. Contrats et écosystèmes agricoles : des mesures agroenvironnementales aux paiements pour services environnementaux. Droit de l'environnement, 237, 296.

Hill S.B., 1985. Redesigning the food system for sustainability. Alternatives, 12, 32-36.

Le Mouël C., De Lattre-Gasquet M., Mora O. (eds.), 2018. Land Use and Food Security in 2050: A Narrow Road. Agrimonde-Terra, Éditions Quæ.

Mandato S., de Vries H., Mayer-Laigle C., 2018. Innovation et tradition : le point de vue des PME françaises de la filière blé dur. Systèmes alimentaires/Food Systems, 3, 245-257.

Pöyry, 2011. Biomass imports to Europe and global availability. VGB-Forschungsstiftung, 29.

Sijtsema S.J., Onwezen M.C., Reinders M.J., Dagevos H., Partanen A., Meeusen M., 2016. Consumer perception of bio-based products: an exploratory study in 5 European countries. NJAS - Wageningen J. Life Sci., 77, 61-69.

Sörqvist P., Hedblom D., Holmgren M., Haga A., Langeborg L., Nöstl A., Kågström J., 2013. Who needs cream and sugar when there is eco-labeling? Taste and willingness to pay for "eco-friendly" coffee. PLoS One, 8, e80719.

Sproul T.W., Kropp J.D., Barr K.D., 2015. The pricing of community supported agriculture shares: evidence from New England. Agric. Finance Rev., 75, 313-329.

Tacconi L., 2012. Redefining payments for environmental services. Ecol. Econ., 73, 29-36.

Vries H. de, Mikolajczak M., Salmon J.-M., Abecassis J., Chaunier L., Guessasma S., Lourdin D., Belhabib S., Leroy E., Trystram G., 2018. Small-scale food process engineering: challenges and perspectives. Innov. Food Sci. Emerg. Technol., 46, 122-130. 


\section{La transition agroécologique de l'exploitation agricole}

LA TRANSITION AGROÉCOLOGIQUE EST UNE TRANSFORMATION SYSTÉMIQUE de notre agriculture et de notre alimentation (Duru et al., 2015). Elle fait appel à des changements de pratiques ainsi qu'aux valeurs qui les régissent pour une large diversité d'acteurs. Elle nécessite donc des « structures sociétales et interactions nouvelles qui supposent des changements de valeurs et de comportements » (d'après OECD, 2010). Du point de vue économique et social, il s'agit d'une transition en train de se faire (transition in the making) au sein de notre régime capitaliste et non d'une révolution. Les principes capitalistes sous-jacents ne sont en effet pas systématiquement remis en cause (Hinrichs, 2014), bien qu'ils soient à l'origine de modes de production non durables (Gorz, alias Bosquet, 1977).

Il existe une pluralité de modèles pour une transition qui se construit « chemin faisant ». La transition agroécologique est une transformation qui se caractérise par une incertitude sur ce à quoi elle va aboutir (Lubello et al., 2017). Cette incertitude est renforcée, d'une part, par l'ambiguïté sur la pertinence des modèles divers qui voient le jour, en matière de forme d'agriculture, de profil de collectifs de travail, de lien avec les filières aval (collecte, transformation, commercialisation...) et les consommateurs, et, d'autre part, par la façon de juger de cette pertinence (Plumecocq et al., 2018).

Peu de travaux portent sur la transition de l'exploitation agricole en tant qu'objet de recherche. La transition est étudiée à l'échelle du territoire, par exemple pour protéger une ressource en eau (Bui et al., 2016), ou à celle d'un secteur d'activité, comme dans le cas du développement d'une filière pour valoriser les légumineuses (Magrini et al., 2016). L'exploitation agricole, pourtant centrale, est négligée (Chantre et al., 2014). La transition agroécologique de l'exploitation agricole nécessite une transformation radicale de la façon de penser le système de production. L'agriculteur doit abandonner la vision linéaire classique d'un système de production qu'il pourvoit en intrants pour produire des denrées alimentaires. À cette vision se substitue une représentation selon laquelle ces denrées alimentaires sont le produit du bon fonctionnement d'un agroécosystème. L'enjeu est alors de restaurer et de piloter cet agroécosystème de façon à valoriser les potentialités locales et à gérer la complexité et les incertitudes de cet agroécosystème (incomplétude des connaissances par exemple sur le fonctionnement du sol, les effets des aléas climatiques, etc. ; Magrini et al., 2019).

Ceux qui étudient ou accompagnent la transition des exploitations agricoles s'attachent à la question du " changement » qu'il faut comprendre et outiller. Le changement comporte en réalité une multiplicité de dimensions : l'engagement et la persévérance des acteurs (motivation, apprentissage, gestion du risque...) ; la confrontation à des freins 
techniques, organisationnels, cognitifs, idéologiques, en relation avec l'environnement social, technique et écologique de l'exploitation agricole ; la redéfinition de ce qui a de la valeur (types de performances, propriétés attendues) ; les nouvelles modalités de pilotage (informations, seuils d'intervention) ; la coconception avec les agriculteurs pour accompagner les changements de leurs modes de raisonnement ; l'évaluation ex ante, ex post, ou chemin faisant.

La mise en œuvre de l'agroécologie nécessite un changement de modes de raisonnement pour piloter l'agroécosystème sur la base des processus écosystémiques. Ces modes de raisonnement sont très différents de ceux basés sur l'utilisation et la transformation d'intrants en produits agricoles, car ils reposent sur un apprentissage par essai-erreur. Les solutions deviennent singulières du fait d'une adaptation du système de production à son contexte de production, en intégrant l'incertitude (connaissances incomplètes, effet des actions...). Il en résulte que la visée et la trajectoire de la transition agroécologique d'une exploitation agricole se raisonnent localement et chemin faisant. Cette configuration modifie la situation de conseil, dans laquelle la dimension collective prend le pas sur l'interaction classique de conseiller à agriculteur. L'organisation même du conseil agricole s'en trouve renouvelée.

Si aujourd'hui le changement est surtout pensé par les chercheurs sous l'angle d'une modélisation de la décision et d'une adoption d'innovations technologiques, la transition agroécologique de l'exploitation agricole invite à produire les connaissances nécessaires pour analyser et accompagner (in the making) les changements techniques, organisationnels et les modes de raisonnement des acteurs qu'elle implique (agriculteurs, conseillers, formateurs...) lors de la reconception des systèmes.

\section{Des avancées scientifiques récentes}

\section{L'importance du contexte sociotechnique et de son déverrouillage}

Dans les travaux conduits sur la transition, l'exploitation agricole est considérée comme enchâssée dans un système sociotechnique et un système de développement agricole. Sa transition agroécologique passe donc par une reconfiguration de ces systèmes dominants à l'échelle territoriale. Ce « régime dominant » correspond ici à l'agriculture conventionnelle qui résiste (Geels, 2004). Les traditions et les multiples interdépendances entre les composantes techniques et sociales du système se sont renforcées au fil du temps, ce qui limite la créativité des acteurs. Une approche systémique est donc nécessaire pour raisonner l'innovation aux différents niveaux qu'elle engage (Meynard et al., 2017) : par exemple, adopter une nouvelle culture dans une rotation nécessite de penser ses fonctions, mais aussi d'accompagner sa maîtrise technique et sa valorisation. L'approche multi-niveaux décrit un monde sociotechnique selon trois niveaux : la niche, le régime 
(dominant) et le paysage (politique, économique...). Proposée par Geels (2004) et déclinée au sein du secteur agricole ces dernières années (Magrini et al., 2016), elle offre un cadre d'analyse pour penser le déverrouillage et favoriser l'innovation.

\section{La transition agroécologique engendre de nouveaux objets de gestion}

L'étude des trajectoires de transition agroécologique met en exergue de nouveaux objets de gestion (Coquil et al., 2014). Ces objets de gestion correspondent aux nouvelles catégories qui apparaissent et autour desquelles le travail va s'organiser : la santé (des plantes, des animaux et des écosystèmes) et non plus les maladies ; l'agrobiodiversité ; la complémentarité entre différentes productions (cultures et élevage, maraîchage et arboriculture, ovins, bovins, agroforesterie, etc.) ; les éléments non productifs d'intérêt écologique et leur rôle dans l'agroécosystème (haies, bois, mares, murets...). Ces changements façonnent également des propriétés attendues des systèmes agricoles comme la résilience, l'efficience, l'autonomie ainsi que des conditions de vie au travail favorables au bien-être des personnes.

\section{Une innovation technologique utile à la transition agroécologique de l'exploitation agricole}

L'innovation technologique, si elle n'est pas le moteur de la transition, n'en est pas moins un élément majeur, notamment :

- la sélection végétale ou animale permet d'accroître l'offre et l'efficience des services écosystémiques, par exemple par la fixation de l'azote ou la couverture du sol, ou par l'utilisation de races locales valorisant la diversité génétique de façon à favoriser la robustesse des systèmes ;

- la robotique permet de réduire le temps de travail des agriculteurs affecté à des activités laborieuses, les aidant dans leurs tâches, par exemple par le développement d'exosquelettes en maraîchage pour soulever sans peine des cagettes, ou bien par le désherbage automatique et mécanique des cultures ;

- les technologies de la communication et de l'information offrent de nouvelles façons de gérer les connaissances. Elles aident, par exemple, des agriculteurs à formaliser leurs connaissances empiriques, à les discuter et à les rendre disponibles au sein d'une communauté plus large grâce à des applications comme $\mathrm{GECO}^{25}$. Cette dernière comprend deux espaces : un espace forum, dans lequel les agriculteurs échangent sur leurs problèmes et les solutions qu'ils mettent en œuvre, et un espace wiki, dans lequel des experts valident et mettent en forme les connaissances échangées sur le forum pour les rendre accessibles à tous.

25. Voir https://geco.ecophytopic.fr/. 


\section{Le partage d'expériences, moteur de la transition agroécologique de l'exploitation agricole}

La transition agroécologique implique que l'agriculteur mobilise et développe ses capacités d'apprentissage pour des pratiques mal connues et en rupture avec le régime dominant. Il conçoit et teste des pratiques alternatives en situation pour en tirer des enseignements (Chantre et al., 2014). De nouvelles normes et références professionnelles découlent de ces transformations de pratiques (Meynard et al., 2017). Cela se fait souvent au sein de collectifs, accompagnés ou non de conseillers et/ou d'animateurs (Chantre et al., 2014 ; Coquil et al., 2014). Ces groupes d'échanges facilitent la créativité, l'apprentissage, la réassurance face aux incertitudes, la construction de nouveaux référentiels d'action, mais aussi la réflexivité et l'appropriation de nouvelles valeurs (Plumecocq et al., 2018). Ils créent un mode de production de connaissances fondé sur les échanges entre tous ceux qui produisent des connaissances jugées pertinentes, y compris, au premier chef, les agriculteurs eux-mêmes.

\section{De l'action collective à la capacité d'action individuelle}

Les travaux conduits sur le déverrouillage sociotechnique et l'apprentissage social se sont focalisés sur la dimension collective de la transition agroécologique. Certains auteurs pointent désormais la nécessité de travailler sur le passage à l'acte de l'individu qui conduit à la transformation de l'exploitation. L'aide à la décision par des processus d'optimisation - approche dominante en sciences agronomiques - ne correspond pas aux besoins d'accompagnement du changement radical et singulier entrepris par les agriculteurs engagés dans une transition agroécologique. Les concepts de " holon ${ }^{26}$ » ou d' " agentivité ${ }^{27}$ » ou les approches relationnelles (Darnhofer et al., 2016) mettent en exergue la capacité d'action vue comme une interaction entre la rationalité de l'acteur et ses valeurs d'une part, et les opportunités et les résistances offertes par l'environnement d'autre part. Cette capacité d'action s'accompagne d'une capacité adaptative définie comme l'aptitude à concevoir et à mettre en œuvre des adaptations ou des changements et à gérer de nouvelles situations sans compromettre de futures options (Nelson et al., 2007).

Outre les travaux conduits sur la conversion à l'agriculture biologique, des travaux récents s'intéressent aux motivations des agriculteurs à s'engager dans la transition agroécologique. Celles-ci apparaissent autant extrinsèques (opportunités liées à une demande du marché) qu'intrinsèques (volonté de respecter la nature) (Plumecocq et al., 2018). La perception du risque vient moduler ces motivations (Bouttes et al., 2018). En effet, la transition apparaît risquée car incertaine et complexe (Duru et al., 2015). Pour se lancer, les agriculteurs évaluent les compromis entre les facteurs externes, tels que les exigences de qualité des produits, les réglementations et les prix, et les exigences internes, telles que les risques liés aux nouvelles techniques de production (Bouttes et al., 2018).

26. Holon : le fait que quelque chose puisse être à la fois système et sous-système d'un système plus vaste (Bland et Bell, 2007).

27. Agentivité : capacité d'agir libérée de ce qu'impose la structure qui nous prédétermine (Wilber, 2001). 


\section{Un cadre conceptuel pour articuler différentes approches de la transition agroécologique}

La transition agroécologique de l'exploitation agricole pose des problèmes méthodologiques car il s'agit de rendre compte, pour la gérer, de la dynamique d'un système complexe dans un environnement changeant avec une forte ambiguïté et une forte incertitude. L'ambiguïté est liée au fait que les valeurs changent au cours de la transition ; ainsi ce qui était acceptable hier devient aujourd'hui ou demain inacceptable. L'incertitude est, quant à elle, constitutive d'une activité stratégique se projetant dans le futur. Elle est inhérente au fonctionnement des systèmes complexes et à leurs propriétés émergentes. Elle est aussi liée à l'incomplétude des connaissances. Cette incertitude pour partie irréductible crée des situations indéterminées dans lesquelles un agriculteur sait qu'il ne peut plus continuer à l'identique, sans pour autant savoir comment agir autrement. Trouver des solutions à ces situations problématiques oblige les chercheurs de différentes disciplines à collaborer dans des approches transdisciplinaires avec les acteurs (Hazard et al., 2018). Différentes postures de recherche coexistent, comme :

- l'analyse des transitions sociotechniques : observer et analyser les transitions ayant eu lieu ou en cours ;

- la modélisation et les jeux sérieux : représenter les exploitations agricoles et explorer leurs évolutions; expliciter des rationalités et tester de nouveaux agencements pratiques;

- la recherche-action : participer à la transformation pour comprendre.

Martin et al. (2018) proposent un cadre conceptuel pour articuler ces trois types d'approches de la transition agroécologique et produire une compréhension des transitions plus complète et utile pour concevoir leurs modes d'accompagnement par les politiques publiques ou les praticiens (figure 4).

Figure 4. Complémentarité entre trois stratégies de recherche sur l'étude de la transition agroécologique des exploitations agricoles (Martin et al., 2018).

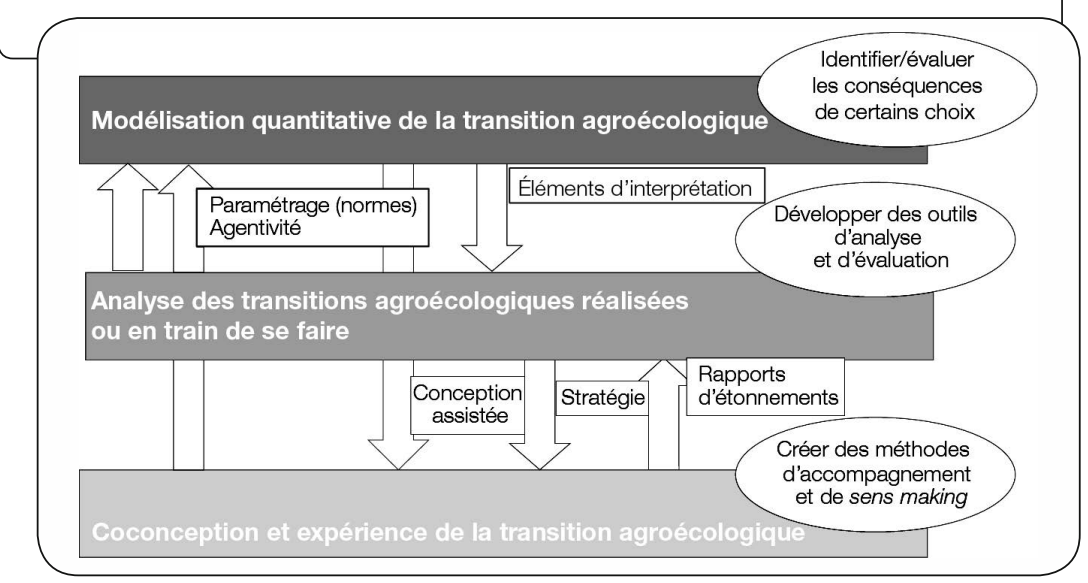




\section{Quelques exemples}

\section{I Évolution de la vulnérabilité des élevages laitiers au cours de leur conversion à l'agriculture biologique}

Ce projet a été conduit de 2005 à 2018. Des éleveurs laitiers conventionnels font face à un contexte incertain et changeant, comme illustré par la crise aiguë du prix du lait consécutive à la fin des quotas laitiers, ou par la fréquence et l'intensité croissante des aléas climatiques. Pour sortir de situations économiques délicates et de la vulnérabilité qui en découle, nombre d'entre eux ont fait le choix d'une conversion à l'agriculture biologique, vue comme une alternative prometteuse avec des prix du lait hauts et stables payés aux éleveurs, soutenus par un marché en croissance. Cependant, s’intégrer dans cette filière nécessite des changements de la part des éleveurs, dans leurs valeurs, leurs pratiques, leurs rapports sociaux sur la ferme et avec l'extérieur, la commercialisation, etc., qui peuvent être source d'incertitudes, notamment dans les premières années de conversion ( 1 an et demi à 2 ans), sans valorisation immédiate du lait au prix de la filière biologique. Ce qui amène à s'interroger sur l'évolution de la vulnérabilité des élevages lors de leur conversion à l'agriculture biologique, c'est-à-dire leur capacité à faire face, à s'adapter ou à surmonter des effets de divers aléas pendant et à l'issue de la conversion à l'agriculture biologique.

Dans ce contexte, le travail de thèse de Maëlys Bouttes, conduit en partenariat avec des acteurs du conseil agricole en Bretagne ${ }^{28}$ et en Aveyron ${ }^{29}$, a eu pour but de caractériser ce type d'évolution et d'identifier les stratégies de conversion limitant la vulnérabilité des élevages. Ce travail a reposé sur des enquêtes en élevage lors de la dernière année de production laitière conventionnelle jusqu'au début de la production biologique (soit entre 3 et 5 ans selon les cas). Des données ont été collectées sur l'évolution des structures (surfaces, tailles de troupeau, etc.), des pratiques (gestion du pâturage, complémentation, etc.), des performances (productivité laitière, résultats économiques, etc.) et de la satisfaction des éleveurs. Pendant la conversion, la vulnérabilité perçue par ces éleveurs a diminué sur les plans économique, agronomique, zootechnique et social, quelles que soient les pratiques mises en œuvre. Ainsi, une éleveuse témoignait : « J'ai le sentiment d'être plus en phase avec ce que veut la société, d'être plus sereine pour l'avenir même s'il y a aussi des risques. " Aucun des éleveurs n’a mentionné que des perceptions négatives ou neutres, et seulement $6 \%$ des réponses révélaient de l'insatisfaction en lien avec des problèmes agronomiques, zootechniques ou liés aux conditions de travail qui n’avaient pas été anticipés. La vulnérabilité évaluée par des indicateurs technicoéconomiques a également diminué lors de la conversion, avec des améliorations de la

28. Avec la Fédération régionale des agriculteurs biologiques (FRAB) et le Groupement d'agriculteurs biologiques de Côtes-d'Armor (GAB22).

29. Avec la Chambre d'agriculture et l'Association pour la promotion de l'agriculture biologique en Aveyron (APABA). 
rentabilité des élevages (la productivité passant en moyenne en Bretagne de $32000 €$ par travailleur et par an avant la conversion à $42000 €$ après la conversion). Cette réduction est permise par des stratégies plus économes et plus autonomes basées sur une utilisation efficace des prairies par le pâturage. Aussi, les différences de vulnérabilité entre élevages sont principalement dues aux différences de pratiques d'éleveurs, soit dans leurs situations initiales, soit dans l'ampleur des transitions effectuées.

Ce travail a montré que les marges de réduction de la vulnérabilité des élevages laitiers permises par la conversion à l'agriculture biologique sont importantes, à condition que cette conversion repose sur une transition franche vers une stratégie plus économe et autonome basée sur une utilisation efficace des prairies par le pâturage. Une série de vidéos de témoignages d'éleveurs a été produite à partir de ces résultats pour sensibiliser aux impacts potentiels d'une conversion à l'agriculture biologique en élevage laitier et aux stratégies requises pour en bénéficier.

\section{Transition professionnelle vers la polyculture-élevage économe et autonome}

Ce projet a été initié en 2009. Son enjeu est d'identifier les ressources (cognitives, matérielles, didactiques) intéressantes pour des agriculteurs souhaitant développer une agriculture plus économe et autonome, mettant à l'épreuve des régulations biologiques et des pratiques qualifiées d'agroécologiques. Les questions posées sont les suivantes : comment la transition agroécologique a-t-elle été initiée chez les agriculteurs du Réseau agriculture durable ? Comment s'est-elle déroulée?

Ce travail a mobilisé les sciences agronomiques et l'ergonomie. Il est basé sur l'analyse des transitions professionnelles de 20 agricultrices et agriculteurs travaillant dans 9 fermes de polyculture-élevage économes et autonomes adhérant au Réseau agriculture durable et de 17 expérimentateurs travaillant sur le dispositif expérimental d'Aster-Mirecourt, où se pratique aussi une polyculture-élevage économe et autonome. Ces agriculteurs, agricultrices et expérimentateurs avaient pratiqué antérieurement la polyculture-élevage basée sur la mobilisation des intrants.

L'accès à l'impensable, la prise de conscience du décalage entre valeurs et pratiques, les difficultés pratiques ou financières ou encore la volonté d'expérimenter sont autant de facteurs d'initiation, souvent interagissant, de la transition professionnelle vers la polyculture-élevage économe et autonome. Les souhaits relatifs au futur des agriculteurs évoluent chemin faisant durant la transition. Les connaissances et les savoir-faire qu'ils mobilisent lorsqu'ils travaillent avec des intrants sont, en partie, inutilisables en situation économe. Leur travail et l'objet sur lequel ils travaillent ne sont plus les mêmes. Le processus de transition s'apparente à un dialogue avec la situation : ainsi, les agriculteurs confrontent leurs souhaits à la réalité et tentent de résoudre les problèmes qui émergent durant la transition par la recherche de solutions efficaces. Ce processus est outillé et stimulé par le recours à des ressources, par exemple la méthode de pâturage 
tournant, les diagnostics, un herbomètre, qui permettent à l'agriculteur de résoudre des difficultés, mais aussi de découvrir de nouvelles possibilités.

Ce projet a donné lieu à deux financements Casdar ${ }^{30}$ (compte d'affectation spécial « Développement agricole et rural »). L'un d'eux, TransAE, est centré sur l'accompagnement de la transition professionnelle des agriculteurs, animateurs, enseignants et chercheurs vers l'agroécologie : il part du postulat que la transition professionnelle de l'agriculteur, pour développer des façons de faire et de penser agroécologiques, implique également une transformation des modes de travail des personnes qui orientent le travail de l'agriculteur au cours de sa carrière. TransAE est un dispositif de recherche-action centré sur l'accompagnement des transformations du travail d'environ 60 agriculteurs et la formation d'élèves de lycée agricole : en quoi l'accompagnement de ces transformations, dans le cadre de la transition agroécologique, questionne-t-il le travail des animateurs, des enseignants et des chercheurs ? TransAE est conduit selon des méthodes favorisant la réflexivité des participants au projet, dans des espaces ouverts au partage et à l'apprentissage collectif. Ces travaux portant sur la créativité des agriculteurs-éleveurs en transition agroécologique sont mis en action dans un dispositif de partage d'expériences impliquant les expérimentateurs d'Aster-Mirecourt ; les savoir-faire, les sens et les sensations mobilisés pour travailler dans ces systèmes y sont convoqués et discutés.

\section{Articuler projet collectif et individuel pour accompagner la transition agroécologique des élevages ovin-lait}

Ce projet a été conduit de 2015 à 2018. La transition agroécologique d'une exploitation agricole nécessite une adaptation des principes de l'agroécologie à son contexte environnemental, économique et social. Au cours de ces dernières années, les travaux conduits sur l'accompagnement des transformations sociotechniques de l'agriculture ont montré d'une part l'importance de l'apprentissage social pour élaborer et/ou contextualiser les connaissances nécessaires à cette transition (Cristofari et al., 2018), et d'autre part l'importance de construire avec les agriculteurs les outils dont ils se serviront pour mener à bien leur propre changement (Cerf et al., 2012). Le travail proposé ici intègre ces deux aspects. Il se situe dans la zone de Roquefort, où les systèmes ovin-lait poursuivent leur intensification et leur agrandissement. Face à ce constat, l'Association des vétérinaires et éleveurs du Millavois (AVEM, créée il y a quarante ans à la suite des luttes sur le causse du Larzac) a souhaité engager une transition agroécologique des fermes sur son territoire. Avec l'aide des vétérinaires, des agronomes et d'un chercheur, les éleveurs du conseil d'administration ont rédigé le projet Salsa (Systèmes agroécologiques laitiers du Sud-Aveyron) retenu à l'appel d'offres Casdar « Mobilisation collective pour l'agroécologie ». Ce projet a ainsi permis aux éleveurs, aux vétérinaires et à leurs partenaires (Parc régional naturel des Grands Causses, INRAE, Centre d'études des techniques agricoles, CETA «De l'herbe au lait », lycée agricole de Saint-Affrique) d'élaborer un outil de diagnostic agroécologique des fermes dans le but

30. PraiFacE 2011-2014; TransAE 2016-en cours en 2019. 
d’identifier les pratiques vertueuses à implémenter dans les fermes. L'hypothèse des éleveurs était que l'autonomie fourragère et énergétique des élevages permettrait de réduire simultanément les charges et l'impact environnemental de l'exploitation. Les partenaires devaient concevoir et mettre en œuvre une méthode de suivi et d'accompagnement des changements dans les fermes. Les chercheurs d'INRAE, dont Camille Lacombe au cours d'un travail de thèse, ont participé aux différentes étapes de ce projet.

L'élaboration de l'outil de diagnostic a demandé des allers et retours entre une conception en salle et sa mise à l'épreuve dans les fermes de l'AVEM. De nouveaux critères proposés par les éleveurs ont été intégrés afin de prendre en compte d'autres dimensions que l'autonomie fourragère et l'autonomie énergétique pour évaluer les pratiques d'élevage. Ils ont souhaité élargir le diagnostic aux dimensions économiques et sociales. Le sens même donné à l'action collective a été mis en discussion : des visions de l'agroécologie plus tournées vers l'emploi local et la vente directe ont été débattues, ce qui a aussi favorisé une adhésion des éleveurs au projet de transition agroécologique. Les éleveurs se sont aussi saisi des premiers diagnostics pour comparer et discuter leurs stratégies et pratiques individuelles. Afin de consolider un usage de l'outil de diagnostic comme support d'échanges entre éleveurs, des groupes de travail entre éleveurs voisins ont été créés pour que ces derniers puissent travailler et échanger sur leurs projets individuels de transition agroécologique. Au cours de ces journées, l'outil de diagnostic a été utilisé comme support d'échanges entre éleveurs, support d'explicitation des stratégies individuelles, outil de suivi ou de simulation de pratiques.

L'outil initialement pensé pour prescrire de bonnes pratiques s'est ainsi transformé au cours de l'action en outil heuristique, c'est-à-dire en une forme d'aide pour penser le changement. N'étant plus producteur d'une norme sur laquelle s'aligner, il permet aux éleveurs de penser la reconception de leur propre système. Il permet le choix d'un projet collectif de transition agroécologique. Il aide les éleveurs à réfléchir sur leurs propres stratégies de changement de pratiques et plus globalement sur leur projet. L'outil devient une forme de médiation entre projet collectif à l'échelle du territoire et projets individuels des éleveurs sur leur ferme.

\section{Les questions de recherche}

\section{Comment analyser la transition agroécologique d'une exploitation agricole?}

Pour évaluer les performances des systèmes agricoles, les sciences agronomiques ont jusqu'à ce jour privilégié l'étude de systèmes stables, dans lesquels les pratiques mises en œuvre sont peu ou pas modifiées au fil des ans. La problématique de la transition était alors réduite à la conception de l'exploitation agricole souhaitée, et le chemin pour l'atteindre était considéré comme relevant des organismes et des agents du développement 
agricole. Pour une transition agroécologique, l'adéquation entre fins et moyens est régulièrement reconsidérée ; elle devient une problématique de recherche en soi pour laquelle il convient de produire des cadres interprétatifs.

Dans la tradition des farming systems, définis comme un ensemble de fermes ayant des points communs (Darnhofer et al., 2012), on peut structurer ce travail de recherche autour de trois tâches.

1. Identifier des systèmes en transition, ce qui constitue une " traque » aux innovations. Il s'agit de mettre au point des méthodes pour identifier les innovations, mais aussi pour saisir leur possible remobilisation dans la conception de trajectoires de transition (outscaling) et les conditions de cette remobilisation. Cette question est traitée notamment par le centre de ressources Initiative for Design in Agrifood Systems (Ideas) ${ }^{31}$ en Île-de-France.

2. Analyser l'évolution conjointe du contexte, des pratiques, des propriétés et des performances des systèmes en transition, afin de caractériser les conditions requises et les facteurs favorables, ou au contraire les effets de verrouillage dans les activités de l'exploitation et de l'exploitant (changements techniques, insertion dans des réseaux, processus d'apprentissage, changements dans les modes de commercialisation et dans l'organisation du travail). C'est un travail interdisciplinaire sur des transitions en cours. Ces travaux nécessiteraient deux types d'approfondissements :

- d'une part, élaborer les concepts et les méthodes couplant le cadre de la durabilité avec des cadres d'analyse de la dynamique des systèmes (résilience, vulnérabilité, efficience, viabilité notamment) pour analyser l'évolution des performances lors de la transition. Le réseau Resilience Alliance ${ }^{32}$ fondé autour du concept de système socio-écologique travaille de manière interdisciplinaire à la déclinaison des concepts de résilience et de vulnérabilité pour les systèmes agricoles ;

- d'autre part, élaborer les concepts et les méthodes pour analyser les transformations du travail (organisation des activités, évolution de la charge mentale liée au pilotage de la complexité et de l'incertain, transformation des mondes professionnels de référence). Il s'agit d'analyser comment la charge de travail oriente les trajectoires de transition agroécologique. Il est clair que les agriculteurs pressurés en travail chercheront la mise au point de systèmes agroécologiques robustes et simples, pas trop demandeurs en travail. Cette quête n'est pas suffisamment prise en compte dans la conception de systèmes innovants, dont la complexité sera vraisemblablement accrue et nécessitera donc une charge de travail et une charge mentale très importantes. Cette question du travail a été délaissée dans le champ des sciences agronomiques.

3. Caractériser comment les objets de gestion sont remis en question lors de la transition agroécologique d'une exploitation agricole et la façon dont ils se reconfigurent, tels que la gestion de la santé des plantes et des animaux, l'agrobiodiversité ou l'intégration des activités dans les systèmes diversifiés.

31. Voir http://www.versailles-grignon.inra.fr/Toutes-les-actualites/2017-IDEAS.

32. Voir www.resalliance.org. 


\section{Comment modéliser la transition agroécologique d'une exploitation agricole?}

Modéliser la transition agroécologique de l'exploitation agricole nécessite avant tout de pouvoir modéliser l'exploitation agricole. Pour rendre compte du changement, la modélisation doit proposer des méthodes de scénarisation et de simulation opérationnelles. Ainsi, pour limiter les temps de calcul lors de l'interaction avec des acteurs de terrain participant à ces scénarisations, il peut être préférable de se baser sur des modélisations statiques plutôt que dynamiques (grille de production des cultures plutôt qu'utilisation de modèles de croissance), de travailler à la parcimonie par couplage de méta-modèles plutôt que d'utiliser des modèles trop complexes et coûteux en temps de calcul. Enfin, il peut être intéressant de mobiliser d'autres informations provenant d'experts de divers horizons afin de tester des trajectoires de transitions agroécologiques d'exploitations agricoles, par exemple avec des jeux sérieux. Ces jeux sérieux sont ici des jeux de rôle invitant des acteurs à simuler des pratiques de gestion de cette exploitation agricole. Ils permettent le partage de connaissances et l'apprentissage sur la situation de gestion.

Une modélisation de l'exploitation agricole demande des simplifications pour analyser et comprendre son fonctionnement (situation de référence), évaluer des scénarios et mettre en débat des mesures d'accompagnement (innovation technique, changements structurels, politiques publiques...), mais également transférer des connaissances. Plusieurs défis de modélisation se posent lorsqu'on aborde l'exploitation agricole.

De façon générale, la modélisation fonctionnelle de l'exploitation agricole est à la croisée des modélisations des systèmes socio-écologique et sociotechnique. L'exploitation agricole peut être représentée comme un système complexe piloté. Sa modélisation nécessite de s'intéresser aux structures cognitives (buts, plans, préférences) et aux mécanismes par lesquels ces structures interviennent dans les processus de décision des agriculteurs et évoluent dans le temps (adaptation par retour d'expérience). Cette modélisation doit représenter la complexité des structures physiques (parcellaire), des structures sociales (par exemple Groupements agricoles d'exploitation en commun, GAEC) et des options d'organisation du travail, ainsi que la diversité des exploitations agricoles. Il faut s'intéresser à de multiples ateliers (par exemple l'intégration culture-élevage requiert de ne pas se limiter aux grandes cultures, à un atelier de production unique, ou à une unité de travail humain à temps plein, ou à un seul revenu). La modélisation se heurte donc à la capacité des modèles à intégrer les multiples et nouveaux objets de gestion qui émergent des processus de transition agroécologique, et à prendre en compte des propriétés attendues de ces systèmes telles que la résilience.

Un autre défi est de créer des cadres de modélisation qui prennent en compte l'incertitude et l'apprentissage sur le temps long. Comment intégrer par exemple l'essai-erreur pratiqué par les agriculteurs sur leur parcellaire pour tester des innovations agroécologiques? Comment intégrer le cadre conceptuel de la viabilité qui s'intéresse à la dynamique d'un système dans un espace de contraintes ? Quelles évolutions envisager pour des démarches d'optimisation, dans un cadre désormais multicritère ? Comment intégrer les changements 
d'échelle d'espace et de temps ? Comment prendre en compte les propriétés émergentes des modèles quand on couple les échelles, et rendre compte des nécessaires compromis entre résilience, efficience et viabilité des systèmes ?

Le réseau d'acteurs est un élément important de la transition agroécologique des exploitations agricoles. Cela nécessite de proposer un cadre d'étude des situations de gestion incorporant une dimension collaborative. En effet, certains objectifs, tels que maintenir une structure du paysage favorable à la biodiversité locale et aux services de régulation biologiques associés, ne peuvent pas être atteints par des stratégies individuelles ; plusieurs agriculteurs doivent collaborer et coordonner leurs actions. La collaboration peut également porter sur des échanges entre les agriculteurs de produits de leurs exploitations (fourrage, fumier...) ou sur le partage de leurs ressources (machines, travail...). Ce type de problème de coordination mêlant initiatives individuelles et approche collective a été peu abordé, mais semble fondamental en agroécologie, notamment pour la transition agroécologique.

\section{Comment produire des connaissances scientifiques sur l'accompagnement de la transition agroécologique de l'exploitation agricole?}

Accompagner la transition agroécologique des exploitants agricoles pose des questions sur les processus de coconception et l'apprentissage que nécessite le changement. Au-delà des méthodes de créativité, il s’agit de travailler sur la nature des connaissances à produire pour coconcevoir, sur la façon de les structurer, sur l'articulation entre la conception et les différentes formes d'expériences (de celle vécue par l'agriculteur aux essais d'expérimentation analytique qu'il visite). Ce travail engage des approches participatives fondées sur la résolution de problèmes impliquant les agriculteurs et leurs conseillers pour mobiliser leurs savoirs et choisir démocratiquement des orientations de la transition. La méthode consiste alors à analyser le processus de changement en y participant : cette participation procède d'itérations entre l'engagement dans l'action et la transformation des systèmes et processus réflexifs avec les acteurs pour apprendre de l'action engagée. Cela permet une production de connaissances scientifiques tant dans le champ des sciences humaines et sociales que dans celui des sciences biotechniques.

Ce travail peut prendre appui sur l'Association internationale sur les systèmes agricoles ${ }^{33}$, en lien avec les travaux du Cirad ("Systèmes de polyculture-élevage d'Afrique de l'Ouest: impliquer les agriculteurs dans la conception des innovations ») et de Boelie Elzen de l'Université de Wageningen. Il se structure autour de quatre tâches.

1. Créer des méthodes de conception en lien avec la situation d'usage pour proposer des solutions localement adaptées. Cela implique de réduire le fossé qui s'est creusé entre concepteurs et utilisateurs, entre créativité et mise à l'épreuve, entre recherche de

33. International Farming System Association (IFSA), http://ifsa.boku.ac.at. 
solutions et production de sens. Les recherches se sont structurées en île-de-France au sein du centre de ressources Ideas.

2. Développer des modes d'accompagnement de la transition agroécologique, de conduite de projets et de gouvernance (recherche, conseil, formation) en favorisant la participation, les apprentissages croisés, le partage d'expériences et l'investigation scientifique dans l'action. Il s'agit de questionner la relation entre le collectif et l'individuel, après avoir mis l'accent sur l'action collective.

3. Travailler sur les liens entre recherche, conseil et formation en partageant les modes d'investigation qui favorisent l'apprentissage : les méthodes d'enquêtes et d'apprentissage dans l'action renouvellent l'intérêt d'une proximité entre la recherche et l'enseignement qui jusqu'à présent visait surtout à favoriser le transfert des connaissances.

4. Analyser et proposer les indicateurs de gestion, les raisonnements tactiques et stratégiques et leurs interactions pour aider l'agriculteur à réduire la complexité de la conduite des agroécosystèmes et ainsi mieux les piloter.

\section{Le développement d'une nécessaire transdisciplinarité}

LES QUESTIONS DE RECHERCHE DÉTAILLÉES PRÉCÉDEMMENT nécessitent clairement des pratiques de recherche interdisciplinaires, voire transdisciplinaires, c'est-à-dire avec les acteurs. Plusieurs chantiers sont en cours pour :

- travailler sur les liens entre agriculture numérique, modélisation et apprentissage social afin d'accompagner la transition agroécologique des exploitations agricoles ;

- articuler les travaux réalisés dans plusieurs disciplines (figure 4). Ces travaux ont été effectués avec des postures de recherche parfois difficilement compatibles, tant les différences de pratiques entre disciplines peuvent être importantes dans la façon de poser les problèmes, de les traiter, d'administrer la preuve et de présenter les résultats (Hazard et al., 2019) ;

- poursuivre le développement méthodologique des approches transdisciplinaires qui font travailler ensemble des chercheurs et des non-chercheurs. Les écoles-chercheurs telles que « Produire et mobiliser différentes formes de connaissances pour et sur la transformation des systèmes agricoles : regards interdisciplinaires » (2015) et "Sciences participatives » (2018) ainsi que la mission « Sciences en société » soutenue par l'Inra ont lancé la dynamique.

Ces initiatives doivent être étendues à nos partenaires de terrain, car l'essor de ces approches se heurte à la vision persistante d'une science descendante qui doit donner les réponses à leurs problèmes. Les chercheurs comme les non-chercheurs peinent à se détacher de cette pratique très normée de la science pour mettre en œuvre des approches participatives, ce que montrent des expériences comme Co-create à Bruxelles ou les projets des Centres d'initiatives pour valoriser l'agriculture et le milieu rural (Civam). 
L'idée est, d'une part, de consolider les méthodologies d'investigation et de pratiques réflexives mobilisables par des communautés mixtes de chercheurs et d'acteurs de terrain, et, d'autre part, de parvenir à combiner la coproduction d'innovation avec les exigences académiques de la production de connaissances scientifiques.

\section{Références bibliographiques}

Bland W.L., Bell M.M., 2007. A holon approach to agroecology. Int. J. Agric. Sustain., 5, 280-294.

Bouttes M., Darnhofer I., Martin G., 2018. Converting to organic farming as a way to enhance adaptive capacity. Organ. Agric., 1-13.

Bui S., Cardona A., Lamine C., Cerf M., 2016. Sustainability transitions: insights on processes of niche-regime interaction and regime reconfiguration in agri-food systems. J. Rural Stud., 48, 92-103.

Cerf M., Jeuffroy M.-H., Prost L., Meynard J.-M., 2012. Participatory design of agricultural decision support tools: taking account of the use situations. Agron. Sustain. Dev., 32, 899-910.

Chantre E., Le Bail M., Cerf M., 2014. Une diversité de configurations d'apprentissage en situation de travail pour réduire l'usage des engrais et pesticides agricoles. Activités, 11, 3-25.

Coquil X., Béguin P., Dedieu B., 2014. Transition to self-sufficient mixed crop-dairy farming systems. Renew. Agric. Food Syst., 29, 195-205.

Cristofari H., Girard N., Magda D., 2018. How agroecological farmers develop their own practices: a framework to describe their learning processes. Agroecol. Sustain. Food Syst., 42, 777-795.

Darnhofer I., Gibbon D., Dedieu B. (eds), 2012. Farming Systems Research into the 21st century: The New Dynamics, Springer, $490 \mathrm{p}$.

Darnhofer I., Lamine C., Strauss A., Navarrete M., 2016. The resilience of family farms: towards a relational approach. J. Rural Stud., 44, 111-122.

Duru M., Therond O., Martin G., Martin-Clouaire R., Magne M.A., Justes E., Journet E.P., Aubertot J.N., Savary S., Bergez J.-E., Sarthou J.-P., 2015. How to implement biodiversity-based agriculture to enhance ecosystem services: a review. Agron. Sustain. Dev., 35, 1259-1281.

Geels F.W., 2004. From sectoral systems of innovation to socio-technical systems: insights about dynamics and change from sociology and institutional theory. Res. Pol., 33, 897-920.

Gorz A. (alias Bosquet M.), 1977. Écologie et liberté, Éditions Galilée, 116 p.

Hazard L., Cerf M., Lamine C., Madga D., Steyaert P., 2019. A tool for reflecting on research stances to support sustainability transitions. Nature Sustain., DOI:10.1038/s41893-019-0440-X.

Hazard L., Steyaert P., Martin G., Couix N., Navas M.L., Duru M., Lauvie A., Labatut J., 2018. Mutual learning between researchers and farmers during implementation of scientific principles for sustainable development: the case of biodiversity-based agriculture. Sustain. Sci., 13, 517-530.

Hinrichs C.C., 2014. Transitions to sustainability: a change in thinking about food systems change? Agric. Human Val., 31, 143-155.

Lubello P., Falque A., Temri L. (eds), 2017. Systèmes agroalimentaires en transition, Éditions Quæ, 184 p.

Magrini M.B., Martin G., Magne M.A., Duru M., Couix N., Hazard L., Plumecocq G., 2019. Agroecological transition from farms to territorialised agri-food systems: issues and drivers. In: Agroecological Transitions: From Theory to Practice in Local Participatory Design, Springer, Cham., 69-98. 
Magrini M.B., Anton M., Cholez C., Corre-Hellou G., Duc G., Jeuffroy M.-H., Meynard J.-M., Pelzer E., Voisin A.S., Walrand S., 2016. Why are grain-legumes rarely present in cropping systems despite their environmental and nutritional benefits? Analyzing lock-in in the French agrifood system. Ecol. Econ., 126, 152-162.

Martin G., Allain S., Bergez J.E., Burger-Leenhard D., Constantin J., Duru M., Hazard L., Lacombe C., Magda D., Magne M.-A., Ryschawy J., Thénard V., Tribouillois H., Willaume M., 2018. How to address the sustainability transition of farming systems? A conceptual framework to organize research. Sustainability, 10, 1-20.

Meynard J.-M., Jeuffroy M.-H., Le Bail M., Lefèvre A., Magrini M.-B., Michon C., 2017. Designing coupled innovations for the sustainability transition of agrifood systems. Agric. Syst., 157, 330-339.

Nelson D.R., Adger W.N., Brown K., 2007. Adaptation to environmental change: contributions of a resilience framework. Annu. Rev. Environ. Resour., 32, 395-419.

OECD, 2010. Eco-Innovation in Industry: Enabling Green Growth, OECD Publishing, 276 p.

Plumecocq G., Duru M., Debril T., Magrini M.-B., Therond O., Sarthou J.-P., 2018. The plurality of values in sustainable agriculture models: diverse lock-in and co-evolution patterns. Ecol. Soc., 23, 21.

Wilber K., 2001. A Theory of Everything: An Integral Vision for Business, Politics, Science and Spirituality, Shambhala Publications, $189 \mathrm{p}$. 



\section{Valoriser les processus écologiques et hydrobiogéochimiques dans des paysages multifonctionnels}

LE PAYSAgE AGRICOLE EST UNE MOSAÏQUE de parcelles et d'espaces interstitiels, de tailles, de formes et d'arrangements variables dans l'espace, mais aussi dans le temps, au rythme des successions culturales, des activités agricoles et de l'aménagement du territoire. Ces arrangements sont liés aux contraintes des exploitations agricoles en relation avec leur mode de production, aux caractéristiques du milieu en relation avec la distribution des sols et des nappes, mais aussi aux activités non agricoles, en particulier aux interfaces. Ces mosaïques, liées aux dimensions socio-économiques du territoire, s'inscrivent dans une histoire et sont porteuses d'enjeux pour la société en matière de production et d'environnement (Gascuel-Odoux et Magda, 2015).

Quelle peut être la valorisation de la biodiversité, des processus écologiques et hydrobiogéochimiques ? C'est-à-dire de tous les processus biotiques, abiotiques et de leurs interactions qui s'expriment dans des paysages, pour une production agricole durable et multiperformante, plus efficiente, plus résiliente et moins polluante (faibles intrants, faibles fuites dans l'environnement), bénéficiant à l'ensemble des acteurs d'un territoire agricole ? C'est la question traitée dans ce chapitre.

Le terme «multifonctionnel » recouvre quelques fonctions clés qui ont fait l'objet de nombreuses études ces dernières années. Il s'agit de la régulation des bioagresseurs (ravageurs, adventices, maladies), de la pollinisation, du maintien de la biodiversité tellurique et aérienne, de la régulation et de l'approvisionnement en matière de qualité eau-sol-air, du maintien de la production végétale et animale (fertilité des sols, nutrition animale). Les fonctions non strictement agricoles (gestion des espèces envahissantes, conservation de la biodiversité patrimoniale) ne sont pas traitées ici.

L'identification des déterminants de la variabilité spatiale et temporelle des milieux et des processus écologiques qui s'y déroulent, et l'identification des éléments du paysage qui sont supports ou leviers d'actions agroécologiques composent une première étape. Ces éléments du paysage ne sont pas forcément des espaces dédiés à l'agriculture, mais peuvent être des espaces naturels. Des échelles de gestion (parcelle et sa bordure, exploitation, territoire, 
bassin versant) et des échelles écologiques (du centimètre carré au paysage agricole) se combinent, car si l'échelle du paysage est un niveau d'organisation idéal pour mettre en regard ces deux échelles, la prise en compte d'échelles plus fines ou plus larges est parfois nécessaire. La question de la complémentarité de différents leviers, assemblant différents milieux et différents systèmes de production, est primordiale à l'échelle du territoire. La dimension temporelle des processus est importante pour réfléchir à des paysages multifonctionnels résilients. Les paysages agricoles évoluent rapidement dans le temps, et cette évolution est souvent indissociable de la dimension spatiale, sous-tendue par des stratégies spatio-temporelles de déploiement des cultures, des variétés et des habitats semi-naturels. À plus long terme, un enjeu fort est d'anticiper les effets des changements globaux, dont le changement climatique avec ses conséquences sur l'utilisation des terres et l'urbanisation. Des points de vigilance particuliers peuvent apparaître, comme la propagation d'espèces envahissantes et de maladies émergentes.

La question des synergies et des antagonismes entre fonctions écosystémiques est prégnante, et cela à différentes échelles spatiales et temporelles. À l'échelle du paysage, il s'agit notamment d'examiner en quoi la coexistence de systèmes agricoles différents au sein d'un même territoire engendre une synergie de certaines fonctions (recyclage, complémentarités...). La question des arbitrages sociaux entre des fonctions et des services écosystémiques, de leurs déterminants et de leurs conséquences sur la gestion des ressources naturelles est un pan de recherche en expansion.

Les enjeux sociétaux sont, d'une part, d'évaluer dans quelle mesure l'organisation spatiale et temporelle des paysages représente un levier pour leur multifonctionnalité et leur résilience aux changements globaux. Il s'agit par exemple de répondre aux questions suivantes : quelles sont les compositions, les agencements spatiaux et les types de gestion des éléments du paysage ? Quelle est la complémentarité entre les systèmes de production au sein d'un territoire?

Ils sont, d'autre part, de progresser dans la conception de paysages multifonctionnels et résilients. Il s'agit par exemple de répondre aux questions suivantes : quels sont les instruments de politiques publiques susceptibles de promouvoir des paysages multifonctionnels et résilients ? Quel est le potentiel d'une gestion concertée et entre quels acteurs pour obtenir ces paysages ? Quel mode de gouvernance faut-il mettre en place?

Les enjeux de recherche sont d'accroître la connaissance des interactions entre les différents processus biotiques qui sous-tendent les différentes fonctions écosystémiques des paysages agricoles. Il s'agit aussi d'analyser et d'intégrer les interactions entre les processus abiotiques et biotiques, d'accroître notre compréhension des effets de la composition, de la configuration et de la gestion des paysages agricoles sur les processus, les fonctions et services, de mieux comprendre leur dynamique temporelle, notamment pour évaluer la résilience des paysages agricoles. Il s'agit également d'avancer dans la compréhension des déterminants économiques et sociaux de la gestion des ressources abiotiques et biotiques dans les paysages et d'analyser les déterminants des préférences relatives des acteurs vis-à-vis de différents aspects de la multifonctionnalité pour comprendre leurs arbitrages. 


\section{Des avancées scientifiques récentes}

LA CAPACITÉ À RENDRE COMPTE DE LA COMPLEXITÉ de processus biophysiques à différentes échelles spatiales et dans des environnements hétérogènes s'est accrue ces dernières années du fait de différentes avancées.

Des progrès ont été faits pour mieux prendre en compte l'hétérogénéité spatiale et temporelle des pratiques agricoles dans l'étude des processus biophysiques. Les modèles de flux abiotiques dans les paysages se complexifient avec la prise en compte des systèmes de production et des contraintes des exploitations agricoles (par exemple, modèle Casimod' $\mathrm{N}$ des projets sous l'égide de l'Agence nationale de la recherche, ANR, Acassya et Escapade sur la cascade de l'azote ${ }^{34}$ ). La représentation des activités agricoles dans l'étude des processus écologiques à l'échelle des paysages devient plus élaborée, avec notamment la notion d'hétérogénéité cachée, la prise en compte plus explicite des pratiques agricoles allant au-delà de la dichotomie entre agriculture biologique et agriculture conventionnelle. Les études semi-expérimentales à l'échelle des paysages ont pris de l'essor et permettent l'analyse des interactions entre des variables paysagères, des processus écologiques et la production agricole. On note également un intérêt renouvelé pour l'écologie du mouvement, avec des avancées sur la façon dont la structure du paysage impacte les mouvements des organismes d'intérêt comme les arthropodes. De même, la description du rôle fonctionnel d'organismes d'intérêt et de leurs réponses à la configuration du paysage est mieux connue, par exemple le rôle de biocontrôle des oiseaux insectivores en viticulture. L'exposition d'organismes vivants à des contaminants est une dimension qui commence à être intégrée dans l'étude de la résilience des paysages agricoles (Réseau Écotox ${ }^{35}$ ).

Ces évolutions se sont accompagnées de la mise en œuvre d'outils qui ont augmenté notre capacité à appréhender le fonctionnement des paysages. Par exemple, les méthodes de métagénomique du sol en routine identifient désormais les effets de l'usage des sols sur la distribution spatiale des communautés telluriques (Ranjard et al., 2013). De nouveaux outils moléculaires permettent aussi de reconstituer les réseaux trophiques des agroécosystèmes et d'étudier la réponse structurelle et fonctionnelle de réseaux d'interaction à des gradients paysagers (Bohan et al., 2013). On peut enfin citer les récentes simulations de paysages virtuels, avec des outils qui visent à s'affranchir en partie de l'expérimentation et d'identifier de nouvelles stratégies de gestion des assolements, prenant en compte à la fois les processus biophysiques et les contraintes socio-économiques des acteurs (Poggi et al., 2018).

Récemment, des connaissances génériques ont été acquises sur le fonctionnement écologique des paysages. La publication de plusieurs méta-analyses sur les effets de l'organisation des paysages sur la régulation des bioagresseurs (par exemple, Veres et al., 2013) met en évidence le rôle majeur des habitats semi-naturels et de l'hétérogénéité spatiale et temporelle des mosaïques de cultures dans le paysage. Des travaux

34. Voir https://www6.rennes.inra.fr/umrsas/Productions/Outils/TNT2-CASIMOD-N-CSAM.

35. Voir https://www.recotox.eu/. 
ont identifié les déterminants paysagers des communautés de pollinisateurs, notamment le rôle clé de la distribution spatiale et temporelle de ressources florales.

Globalement, les processus abiotiques sont de mieux en mieux intégrés dans le paysage, en considérant les agroécosystèmes et les milieux dans lesquels ils s'inscrivent, mais souvent limités à un seul élément chimique. De la même façon, si certaines approches considèrent plusieurs processus biotiques dans un même paysage, la plupart des études s’intéressent à un seul processus écologique. Les analyses multifonctionnelles d'un paysage sont souvent basées sur l'utilisation d'indicateurs, de proxy, c'est-à-dire dérivés de liens supposés entre, par exemple, le type d'habitat et des fonctions, plutôt que sur la quantification de processus et de leurs interactions. Il y a quelques exceptions à ce constat, comme les études basées sur les dispositifs Biodiversity Exploratories développés en Allemagne. Dans ces études, on peut dénombrer par exemple 14 types de mesures de diversité et de processus dans les compartiments telluriques et aériens en prairies (Allan et al., 2015).

Dans le même temps, les recherches interdisciplinaires reliant sciences sociales et biophysiques à l'échelle des paysages se sont renforcées. Le cadre des systèmes socioécologiques (Ostrom, 2009) a été largement mobilisé pour documenter les mécanismes et les conditions sociales et écologiques, leurs interrelations et leurs conséquences. Des projets de recherche-action visant à favoriser des actions collectives et des innovations agroécologiques se sont mises en place à l'échelle de territoires (Berthet et al., 2016). L'évaluation économique des services écosystémiques à large échelle s'est fortement accrue, en Europe comme ailleurs. On citera ici la contribution de lan Bateman et de son équipe, avec des déclinaisons particulièrement pertinentes sur l'évaluation du service de pollinisation à l'échelle de paysages (Bateman et al., 2014). Les progrès dans la spatialisation des activités proposent des analyses coût-efficacité de différents scénarios d'organisation spatiale pour la provision de services écosystémiques. La question de la gouvernance est aussi traitée, par exemple par des analyses du gain à la coopération entre acteurs pour favoriser la fourniture de services écosystémiques. Ces travaux apportent les connaissances de base pour aborder la question des arbitrages sociaux entre services écosystémiques.

\section{Quelques exemples}

\section{Paysage et service de " contrôle biologique "}

Les projets de recherche documentant les effets du paysage sur les auxiliaires, les bioagresseurs et l'intensité du contrôle biologique avec une utilisation en routine de proies sentinelles se sont multipliés. On citera différents projets soutenus par l'ANR (Peerless, Landscaphid, Agrobiose), le projet Biodiversa Farmland ${ }^{36}$ ou le projet européen FP7 PURE. La publication de méta-analyses ou de synthèses bibliographiques portant spécifiquement

36. Voir http://www.farmland-biodiversity.org. 
sur les liens entre paysage et biocontrôle a montré que si les habitats semi-naturels peuvent favoriser le biocontrôle dans les parcelles agricoles, la gestion des espaces agricoles à l'échelle du paysage peut être déterminante, par exemple la proportion de paysage gérée en agriculture biologique (Muneret et al., 2019). Le suivi de dispositifs de réseaux de parcelles chez des agriculteurs indique que les effets du paysage sur le contrôle biologique sont souvent variables parce que généralement modulés par la gestion parcellaire, par exemple les pratiques locales (intensité, etc.) d'usage des pesticides (Ricci et al., 2019). Les suivis menés sur les observatoires des pratiques à long terme dans les zones ateliers ${ }^{37}$ ont permis de mieux appréhender les effets de l'histoire de la gestion agricole des parcelles, de leurs bordures et des paysages sur les dynamiques écologiques. Ces travaux ont été accompagnés de la mise en place de réseaux d'observations à long terme sur des gradients paysagers et d'intensité de pratiques, notamment le Réseau national Sebiopag multicultures ${ }^{38}$ ou le site atelier Biodiversité et viticulture Bacchus ${ }^{39}$.

\section{Paysage et service de " pollinisation "}

La période récente a été marquée par des avancées sur la compréhension de l'écologie de l'abeille domestique, l'importance de la distribution spatio-temporelle des ressources florales pour les pollinisateurs, l'impact des pesticides, notamment les néonicotinoïdes, sur les colonies d'abeilles domestiques et l'importance du service de pollinisation pour les cultures oléagineuses à l'échelle des paysages agricoles. Ces avancées ont été permises par le dispositif Ecobee (Odoux et al., 2014). Le suivi des colonies et la veille palynologique de 50 ruches disposées dans des paysages contrastés ont ainsi permis de mettre en évidence l'importance de la flore adventice, le coquelicot en particulier, pour la survie des abeilles domestiques, ressource indispensable entre les périodes de floraison du colza et du tournesol, et l'impact négatif des néonicotinoïdes sur la colonie. Plus récemment, des expérimentations menées le long de gradients paysagers ont montré que la pollinisation par l'abeille domestique et par des pollinisateurs sauvages peut significativement augmenter les rendements de colza et de tournesol. Ces résultats ont été largement transférés aux agriculteurs et aux apiculteurs grâce notamment au partenariat avec l'Itsap-Institut de l'abeille ${ }^{40}$, et plus largement aux conseillers techniques et enseignants avec le $\mathrm{MOOC}^{41}$ « Abeilles et environnement » lancé en 2019.

\section{I Épidémiologie végétale et animale à l'échelle des paysages}

Depuis une dizaine d'années, les questions de gestion des paysages agricoles pour limiter le risque épidémiologique constituent un enjeu majeur. En épidémiologie végétale, il s’agit

37. Voir https://inee.cnrs.fr/fr/zones-ateliers.

38. Services écosystémiques assurés par la biodiversité dans les paysages agricoles. Voir http://sebiopag. inra.fr/.

39. Voir http://siteatelier-bacchus.com/fr/.

40. Institut technique et scientifique de l'apiculture et de la pollinisation.

41. Massive open online course. 
de gérer la diversité intra et interspécifique des cultures à plusieurs échelles d'espace et de temps en faisant appel à des concepts en épidémiologie, en écologie évolutive théorique et en écologie des paysages (Papaïx et al., 2018). En épidémiologie animale, la prise en compte du paysage est beaucoup plus récente et a surtout porté sur les maladies vectorielles. Le projet européen EDENext ${ }^{42}$, en rassemblant 46 partenaires européens, a eu un effet structurant et permis la mise en œuvre d'outils pour surveiller et gérer les populations vectrices de maladies. Le projet Biodiversa SmallForest présente un volet sur les tiques et la borréliose de Lyme à l'échelle du paysage. Le paysage et ses conséquences sur les maladies à tiques sont au centre du programme Oscar ${ }^{43}$ (Outil de simulation cartographique à l'échelle du paysage agricole du risque acarologique) déployé sur les zones ateliers Armorique et Pygar.

\section{Stratégies de gestion de la ressource en eau dans les territoires}

Cet enjeu a par exemple été porté par une collaboration franco-indienne sur l'adaptation de l'agriculture irriguée dans un contexte de rareté de l'eau et de changement climatique. L'observation s'est appuyée sur le bassin versant agricole de Berambadi, pour lequel l'expansion de l'irrigation, les propriétés des sols ont été renseignées par télédétection ; en parallèle, une typologie des systèmes agricoles a été élaborée et leur vulnérabilité au changement climatique a été évaluée. La plateforme de modélisation Record ${ }^{44}$ a permis de coupler le modèle de culture STICS, calibré pour une diversité de cultures, le modèle hydrologique AmBHAs ainsi qu'un modèle économique et un modèle de décision pour tester des dimensions de gestion adaptative tactique et stratégique. Les simulations suggèrent que des scénarios fondés sur le renchérissement du coût de l'irrigation permettraient de stabiliser la ressource en eau, mais entraîneraient des risques financiers pour de nombreuses exploitations ou imposeraient le retour à une agriculture pluviale de subsistance. Des scénarios prenant en compte une meilleure répartition temporelle de l'irrigation et pénalisant les cultures dont les besoins en eau sont importants durant la saison chaude et sèche apparaissent comme les plus prometteurs. Le projet ANR Atcha ${ }^{45}$, en cours, associe un modèle intégré et une approche participative pour accompagner l'adaptation de l'agriculture au changement climatique dans un réseau de bassins versants expérimentaux du sud de l'Inde.

\section{Stratégies des acteurs et santé des plantes}

Certains projets ont renforcé les synergies entre sciences biophysiques et sciences sociales. Trois projets, donnés ici à titre d'exemple, ont porté sur les modalités et les conditions organisationnelles d'une gestion collective paysagère de la santé des plantes.

42. Voir https://www.edenext.eu/.

43. Voir https://www6.inra.fr/oscar.

44. Rénovation et coordination de la modélisation des cultures pour la gestion des agroécosystèmes.

45. Voir https://www6.inra.fr/atcha/. 
Le projet Fondu s'est intéressé à l'utilisation des fongicides en blé et en viticulture et a démontré que les stratégies les plus durables doivent maximiser l'hétérogénéité de la sélection de souches résistantes dans des échelles de temps et d'espace larges. Le projet montre que les raisonnements ne doivent pas être menés au niveau individuel, mais plutôt à celui d'un territoire.

Le projet Riz éternel, quant à lui, montre que la diversité particulièrement élevée en riz cultivés assure la durabilité des rizières des terrasses du Yuanyang en Chine. Cette diversité repose sur des normes sociales considérant les semences comme un bien commun (Hannachi et Dedeurwaerdere, 2019) dont le maintien ne peut être assuré que si les acteurs mettent en place des mixed-form markets, c'est-à-dire des marchés visant collectivement à la fois des gains économiques et des objectifs socio-écologiques de bien-être non monétaires. On peut observer des processus de déstabilisation visibles par des points de basculement socio-économiques.

La modélisation économique et écologique menée dans le projet Peerless montre que le paysage influence l'efficacité de l'usage des pesticides surtout dans les situations où la production n'est pas intensive. Des travaux identifient la composition optimale des paysages pour un rendement visé ou une maximisation à la fois de la production et des abondances d'auxiliaires avec une utilisation réduite de pesticides.

\section{Les questions de recherche}

\section{Analyser les effets de l'hétérogénéité à des échelles spatiales et temporelles emboîtées}

L'analyse des effets de l'hétérogénéité à différentes échelles d'espace et de temps reste un défi en agroécologie. Cette analyse passe par la compréhension des interactions entre les composantes abiotiques et biotiques, biogéochimiques et écologiques, dans l'espace et le temps. Les fronts de science incluent une meilleure compréhension de la dynamique de populations (par exemple, des vecteurs de maladies), de populations en interaction (pathosystèmes) et de communautés (par exemple, des ennemis naturels de ravageurs) dans des environnements hétérogènes et changeants. Le rôle des interfaces entre milieux cultivés et milieux semi-naturels (refuge, échanges d'organismes entre les deux habitats, etc.) reste peu documenté, que ce soit pour la gestion des maladies, le contrôle biologique ou la pollinisation. Les effets de la diversification agricole sur l'intensité et la stabilité des processus écologiques d'intérêt en agroécologie sont peu connus à l'échelle du paysage. Les effets de concentration et de dilution, de connectivité et de régulation des flux sont encore mal compris. Le développement de modèles multi-organismes à l'échelle de paysages aiderait à mieux comprendre la fourniture de services écosystémiques aux différentes échelles d'espace et de temps. 
Ces différentes questions sont explorées, que ce soit en épidémiologie végétale et animale, en écologie du paysage appliquée au biocontrôle et à la pollinisation (approche d'écologie comparative et d'expérimentation) ou sur les flux d'éléments chimiques (pesticides, nutriments). Les recherches impliquent une communauté de chercheurs dont le point commun est la compréhension de processus biophysiques et écologiques à l'échelle des paysages.

\section{Développer des approches intégrées pour traiter de la multifonctionnalité des paysages}

Les processus abiotiques sont généralement considérés de manière compartimentée, par élément chimique ou par objet du paysage. Peu de processus écologiques sont considérés de façon simultanée. Les flux entre sol, eau et air sont de mieux en mieux couplés, mais cela reste l'exception (cas de l'azote). Les travaux traitant d'un ensemble de flux dans une vision intégrative sont rares. Finalement, la question de la complémentarité ou de la synergie entre différentes fonctions n'est encore que peu abordée. Et cela limite notre capacité à analyser plusieurs questions cruciales en agroécologie, par exemple la question de la coexistence de systèmes de production différents au sein d'un même territoire et des synergies qui s'opèrent entre eux. La réalisation d'études intégrées pour évaluer, à l'échelle paysagère, les impacts des systèmes de production sur les ressources et les organismes reste donc un défi majeur. Les approches de modélisation intégrée, visant un couplage, d'une part, entre des processus pédologiques, hydrologiques et atmosphériques et, d'autre part, entre ces processus et des processus écologiques sont prometteuses. L'étude des interactions entre des flux abiotiques et biotiques reste embryonnaire, mais une réflexion récente a permis la présentation d'un cadre de modélisation (Vinatier et al., 2016). Le recours à la simulation de paysages nécessite des recherches, notamment pour intégrer les rétroactions entre le processus d'intérêt et la structure du paysage, pour prendre en compte la diversité des acteurs, ou pour accroître le réalisme des modèles de décision. Intégrer cette complexité dans un modèle demeure difficile et d'autres pistes peuvent aussi être explorées. Dans ces approches intégrées, il semble essentiel d'associer les sciences économiques et sociales dès l'amont, et non a posteriori.

\section{Rendre compte de la diversité des acteurs dans l'analyse des processus biophysiques à l'échelle des paysages}

La participation des acteurs est un facteur clé à l'échelle du paysage, d'où l'importance des recherches sur les instruments de politiques publiques, les instruments plus volontaires tels les incitations non économiques (nudges) et les processus de construction de cadrage commun et d'apprentissage collectif (community building) entre les acteurs des paysages. L'identification de mécanismes incitatifs permettant la coordination des différents acteurs d'un territoire pour promouvoir une gestion agroécologique du paysage demeure un enjeu majeur. 
On note également que les modèles formels utilisés dans le cadre des systèmes socioécologiques considèrent le comportement humain par un modèle d'acteur rationnel, sans rendre compte de sa complexité. Intégrer cette complexité dans des modèles formels est un front de recherche en sciences de gestion. Relever ce défi implique d'étudier des acteurs et des collectifs d'acteurs en interaction, dans des études interdisciplinaires, incluant une interdisciplinarité en sciences sociales (économie, sciences de gestion, sociologie, anthropologie) pour une meilleure intégration des différents aspects du comportement humain. Il semble également important de privilégier le lien à l'action (learning by doing) pour sortir de l'inertie des collectifs d'acteurs et accompagner la conception de paysages agroécologiques en considérant explicitement les demandes et l'organisation des acteurs.

\section{Accompagner la conception de paysages agroécologiques}

Les paysages sont des échelles collectives, et la mise en place et la gestion de fonctions écologiques à cette échelle implique une mobilisation collective des acteurs. Cette mobilisation peut être renforcée par l'élaboration d'indicateurs et d'outils de décision pour les acteurs des paysages, d'autant plus s'ils sont construits avec eux via des dispositifs de recherche participative ou de recherche-action. De tels dispositifs induisent la sensibilisation et la participation des acteurs, tout en offrant des perspectives de recherches interdisciplinaires novatrices. La modélisation et la simulation de processus biophysiques et écologiques, l'identification de règles de décision par diverses démarches (métamodélisation par exemple), la modélisation d'accompagnement (jeux sérieux...) sont des outils qui peuvent contribuer à la conception de paysages agroécologiques et à des boucles de progrès dans le cadre d'une gestion adaptative. Plusieurs équipes d'INRAE travaillent sur le couplage de modèles analysant la performance des paysages en matière de production, de protection des cultures, de bilan de gaz à effet de serre, de pollinisation et de biodiversité dans le but d'identifier des paysages multiperformants (projet API-SMAL). Les avancées sont fortement tributaires de dispositifs d'acquisition et de gestion de données, mais aussi d'outils de modélisation et de simulation.

\section{Dispositifs et développements méthodologiques}

\section{Dispositifs d'acquisition de données}

L'analyse et la modélisation de processus spatio-temporels tels que ceux en œuvre à l'échelle de paysages ruraux sont souvent confrontées à la difficulté de disposer de jeux d'observations cohérents, spatialement bien répartis et sur des durées significatives. Ainsi, pour la conception ou l'analyse de systèmes agroécologiques paysagers, trois priorités complémentaires sont à noter.

1. La création de sites d'observation et/ou d'expérimentation pluridisciplinaires (experimental landscape ecology). Les infrastructures comme les zones ateliers actuelles peuvent être de bonnes candidates du fait de la documentation existante sur des durées avoisinant souvent une à trois décennies, de consortiums de recherche et développement bien 
rodés. Ces infrastructures nécessitent dans la plupart des cas d'être étendues au plan thématique (biophysique, biotechnique, sciences sociales) et instrumental pour acquérir de manière coordonnée des données pour l'étude de systèmes de production agroécologiques. L'émergence et la pérennisation de « réseaux » de paysages contrastés amènent à identifier de façon harmonisée des situations de productions et des gradients paysagers. Un exemple de cette logique est le réseau national Sebiopag pour l'étude des services écosystémiques assurés par la biodiversité dans les paysages agricoles, qui rassemble des paysages localisés dans cinq régions françaises.

2. Le renforcement de moyens d'observation, notamment de nouveaux capteurs (proxi et télédétection), afin d'accéder à des données de haute densité et de haut débit pour l'ensemble des variables (biotiques, abiotiques et pratiques des acteurs), nécessaires pour l'analyse de la variabilité des phénomènes écologiques et agronomiques à l'échelle paysagère (voir chapitre 6).

3. L'amplification d'approches participatives pour compléter l'observation scientifique par les connaissances expertes d'acteurs ou par des observations non formelles opérées par des acteurs non scientifiques ou professionnels. On pourrait également envisager la mise en place d'expériences économiques à grande échelle pour étudier les mécanismes incitatifs qui encouragent l'enrôlement des agriculteurs dans des mesures agro-environnementales de conception innovante (bonus d'agglomération, engagements collectifs, rémunération au résultat).

\section{Gestion et analyses statistiques des données}

Les méthodes statistiques existantes sont à adapter pour traiter les données hétérogènes. Les modèles à l'échelle des paysages sont éminemment multidisciplinaires (écologie, sociologie, agronomie) et doivent donc considérer une multiplicité de types de données (par exemple comptage, occurrence, mesure spectrale, marque génétique) issues aussi bien des big data, comme des images aériennes ou du séquençage haut débit, que des observations plus ponctuelles souvent obtenues en écologie. Dans ce cadre, les méthodes statistiques classiques ne s'appliquent plus. De nombreux outils sont maintenant utilisés assez couramment en écologie spatiale (régression $\mathrm{PLS}^{46}$ pour les multicolinéarités ou le big data, arbre de décision et forêt aléatoire pour les variables hétérogènes et la prédiction, statistique spatiale via INLA ${ }^{47}$ intégrant les aspects temporels des données, etc.). Cependant, les données actuelles sont de plus en plus spatio-temporelles. Elles demandent donc de caractériser des dépendances dans l'espace et le temps liées aux dynamiques des processus étudiés. Les modèles issus de la statistique spatiale s'étendent progressivement au domaine spatio-temporel. Bien que des solutions sous le logiciel $\mathrm{R}^{48}$ existent, un travail de transfert vers les non-spécialistes est nécessaire. Enfin, ce passage vers le domaine spatiotemporel peut aussi se faire via la modélisation explicite des dynamiques des processus

46. Partial Least Squares.

47. Integrated Nested Laplace Approximations.

48. Voir https://fr.wikipedia.org/wiki/R_(langage). 
étudiés. Ces approches dites « mécanistico-statistiques » sont en plein essor du fait des avancées en statistiques computationnelles (optimisation de problèmes complexes, statistiques bayésiennes, Approximate Bayesian Computation, pattern-oriented modelling).

\section{Approches de modélisation, de simulation et d'extraction de connaissance}

Une difficulté majeure est de trouver un compromis acceptable entre une modélisation trop complexe non gérable et une modélisation trop simpliste ne représentant pas ou mal les interactions aux échelles des paysages. La modélisation en environnement hétérogène s'oriente, d'une part, vers la modélisation intégrée ou ensembliste (couplage de modèles) pour étudier des systèmes complexes et des interactions entre processus, et, d'autre part, vers la méta-modélisation, c'est-à-dire la conception de modèles édictant des règles apprises des simulations faites sur des runs de modèles plus complexes pour mieux adapter la modélisation aux besoins des acteurs. Une autre stratégie est de combiner les deux approches, comme celle choisie dans la plateforme Maelia ${ }^{49}$ qui agrège des connaissances imparfaites, ou comme celle d'études ciblées pour approfondir les connaissances. Dans ces dernières, les liens entre les éléments de connaissances ainsi acquis demeurent in fine difficiles à établir. L'approche expérimentale avec manipulation de paysage et suivi à long terme ou les dispositifs de type réseau de paysages contrastés sont intéressants, car ils répondent aux besoins de calibration et de validation des modèles. La simulation de scénarios permet parfois de repérer des configurations spatiales et/ou temporelles pour une fonction ou pour un ensemble de fonctions visées. Cette approche par simulation permet d'accéder à de nombreuses variables agrégées à différentes échelles de temps et d'espace, utiles aux usagers pour comprendre, analyser, décider. L'exploration des données de simulation demande de nouveaux développements en méthodes informatiques de type fouille de données et apprentissage (Bouadi et al., 2017), mais aussi des avancées dans le domaine de la visualisation et de la requête de données spatio-temporelles.

\section{Références bibliographiques}

Allan E., Manning P., Alt F., Binkenstein J., Blaser S., Blüthgen N., Böhm S., Grassein F., Hölzel N., Klaus V.H., Kleinebecker T., Morris E.K., Oelmann Y., Prati D., Renner S.C., Rillig M.C., Schaefer M., Schloter M., Schmitt B., Schöning I., Schrumpf M., Solly E., Sorkau E., Steckel J., Steffen-Dewenter I., Stempfhuber B., Tschapka M., Weiner C.N., Weisser W.W., Werner M., Westphal C., Wilcke W., Fischer M., 2015. Land use intensification alters ecosystem multifunctionality via loss of biodiversity and changes to functional composition. Ecol. Lett., 18, 834-843.

Bateman I.J., Harwood A.R., Abson D.J., Andrews B., Crowe A., Dugdale S., Fezzi C., Foden J., Hadley D., Haines-Young R., Hulme M., Kontoleon A., Munday P., Pascual U., Paterson J., Perino G., Sen A., Siriwardena G., Termansen M., 2014. Economic analysis for the UK national ecosystem assessment: synthesis and scenario valuation of changes in ecosystem services. Environ. Resour. Econom., 57, 273-297.

49. Multi-Agents for Environmental Norms Impact Assessment. 
Berthet E.T.A., Barnaud C., Girard N., Labatut J., Martin G., 2016. How to foster agroecological innovations? A comparison of participatory design methods. J. Environ. Plan. Mgmt, 59, 280-01.

Bohan D.A., Raybould A., Mulder C., Woodward G., Tamaddoni-Nezhad A., Bluthgen N., Pocock M., Muggleton S., Evans D.M., Astegiano J., Massol F., Loeuille N., Petit S., Macfadyen S., 2013. Networking agroecology. Adv. Ecol. Res., 49, 2-67.

Bouadi T., Coedrier M.-O., Moreau P., Quiniou R., Salmon-Monviola J., Gascuel-Odoux C., 2017. A data warehouse to explore multidimensional simulated data from a spatially distributed agro-hydrological model to improve catchment nitrogen management. Environ. Model. Soft., 97, 229-242.

Gascuel-OdouxC., Magda D., 2015. Gérer les paysages et les territoires pour la transition agroécologique. Innovations agronomiques, 43, 95-106.

Hannachi M., Dedeurwaerdere T., 2019. Des semences en commun pour gérer les maladies. Étude comparative de rizières dans le Yuanyang (Chine). Études rurales, 202, 76-97.

Muneret L., Auriol A., Thiery D., Rusch A., 2019. Organic farming at local and landscape scales fosters biological pest control in vineyards. Ecol. Appl., 29, 1-15.

Odoux J.-F., Aupinel P., Gateff S., Requier F., Henry M., Bretagnolle V., 2014. ECOBEE: a tool for longterm honey bee colony monitoring at the landscape scale in West European intensive agroecosystems. J. Apicult. Res., 53, 57-66.

Ostrom E., 2009. A general framework for analyzing sustainability of social-ecological systems. Science, 325, 419-422.

Papaïx J., Rimbaud L., Burdon J.J., Zhan J., Thrall P.H., 2018. Differential impact of landscape-scale strategies for crop cultivar deployment on disease dynamics, resistance durability and long-term evolutionary control. Evol. Appl., 11, 705-717.

Poggi S., Papaïx J., Lavigne C., Angevin F., Le Ber F., Parisey N., Ricci B., Vinatier F., Wohlfahrt J., 2018. Issues and challenges in landscape models for agriculture: from the representation of agroecosystems to the design of management strategies. Landscape Ecol., 33, 1679-1690.

Ranjard L., Dequiedt S., Chemidlin Prévost-Bouré N., Thioulouse J., Saby N.P., Lelievre M., Maron P.A., Morin F.E., Bispo A., Jolivet C., Arrouays D., Lemanceau P., 2013. Turnover of soil bacterial diversity driven by wide-scale environmental heterogeneity. Nat. Commun., 4, 1434.

Ricci B., Lavigne C., Alignier A., Aviron S., Biju-Duval L., Bouvier J.C., Choisis J.P., Franck P., Joannon A., Ladet S., Mezerette F., Plantegenest M., Roger J.L., Savary G., Thomas C., Vialatte A., Petit S., 2019. Local pesticide use intensity conditions landscape effects on biological pest control. Proceed. Royal Soc. Ser. B., 286, 20182898.

Veres A., Petit S., Conord C., Lavigne C., 2013. Does landscape composition affect pest abundance and their control by natural enemies? A review. Agric. Ecosyst. Environ., 166, 110-117.

Vinatier F., Lagacherie P., Voltz M., Petit S., Lavigne C., Brunet Y., Lescourret F., 2016. An unified framework to integrate biotic, abiotic processes and human activities in spatially explicit landscape models. Front. Environ. Sci., 4, 6. 


\section{Valoriser la diversité génétique en sélection végétale et animale}

UN SYSTÈME AGROÉCOLOGIQUE DOIT CONSIDÉRER la contribution possible de la diversité biologique à tous ses niveaux d'organisation et de fonctionnalité, y compris en incluant la domestication de nouvelles espèces. Or les travaux scientifiques s'arrêtent souvent au niveau taxonomique, c'est-à-dire au niveau de la diversité spécifique et des interactions entre espèces. Réfléchir à l'apport et au rôle de la diversité génétique intraspécifique est un enjeu fort de l'agroécologie.

Il s'agit d'évaluer la contribution de la diversité génétique à la performance des systèmes agroécologiques des cultures et des élevages, de comprendre les mécanismes d'action de cette diversité, ses interactions avec le contexte environnemental, d’identifier les traits d'intérêt majeurs impliqués dans les interactions entre les plantes d'une part, et entre les animaux d'autre part, pour le maintien de la diversité et pour la performance agroécologique de la culture et/ou de l'élevage. Il s'agit in fine de définir la gamme de diversité permettant l'expression des mécanismes favorables à la mise en œuvre de systèmes de production plus résilients.

Ces enjeux de connaissance s'articulent avec des enjeux finalisés, comme celui de définir des critères de sélection, et les gammes nécessaires de variabilité génétique de ces critères pour le fonctionnement agroécologique de l'unité de production. Il s'agit de créer des schémas de sélection qui prennent en compte des objectifs de diversité à l'échelle de l'individu (plasticité), mais aussi du groupe d'individus composant le couvert ou le troupeau, de définir le déploiement de la diversité, en relation avec le service écosystémique attendu adapté au contexte environnemental, c'est-à-dire décliné dans l'espace et dans le temps dans un milieu donné. Pour cela, il faudra lever certains verrous, comme celui de l'évaluation et de l'inscription au catalogue de cultures diversifiées. Prendre en compte une plus grande diversité peut entraîner des difficultés de gestion des systèmes agricoles, augmenter le travail de l'agriculteur ou de l'éleveur, diminuer la capacité à garantir la qualité de produits agricoles, plus hétérogènes, et par là même remettre en cause l'automatisation des processus de transformation et la standardisation attendue pour le produit final. La diversification génétique est donc à évaluer sur l'ensemble de la chaîne, de l'unité de production au produit agricole et alimentaire, en passant par le mode de production. 


\section{Des avancées scientifiques récentes}

LES TRAVAUX CONSIDÉRANT LA CONTRIBUTION de la diversité génétique intraspécifique au développement de systèmes agroécologiques sont récents. Ils ont émergé un peu plus tôt chez les plantes que chez les animaux. Cette approche est explorée et discutée dans des articles de synthèse, chez les plantes en 2015 (Litrico et Violle, 2015), chez les animaux en 2016 (Phocas et al., 2016a, 2016b). Chez les plantes, une seule variété homozygote représente souvent la majeure partie des cultures d'une espèce (le blé par exemple). Chez les animaux, il existe d'ores et déjà une part importante de diversité à l'intérieur même du troupeau, même dans les races fortement sélectionnées (vaches laitières par exemple). Malgré cette différence, les concepts mobilisés et les constats présentent de nombreux points communs entre la sélection végétale et la sélection animale.

\section{Les plantes, les peuplements cultivés}

Chez les plantes, dans les peuplements cultivés, les principales avancées sont la mise en évidence du rôle positif de la diversité génétique, d'une part, sur la stabilité de la production de biomasse et la résistance à la sécheresse (Prieto et al., 2015), d'autre part, sur l'abondance des espèces composant un mélange cultivé (Meilhac et al., 2019).

Prendre en compte cette diversité génétique dans les schémas de sélection est encore peu fréquent dans la littérature récente. Certains projets en cours ou récemment terminés s’appuient sur de la méthodologie ancienne développée pour les mélanges variétaux ou les populations composites pour traiter de ce sujet. Un exemple récent (Sampoux et al., in press) porte sur la sélection d'une association d'espèces, à partir de l'évaluation de trois schémas de sélection, pour juger de l'aptitude à l'assemblage interspécifique :

- un schéma basé sur la sélection de chaque espèce sur sa performance en culture pure ;

- un schéma basé sur la sélection de duos d'espèces sur leur performance en culture mélangée (sélection sur l'aptitude réciproque à l'association, S-ARA) ;

- un schéma basé sur la sélection d'une espèce qui donne de bonnes performances en association avec n'importe quelle autre espèce (sélection sur l'aptitude générale à l'association, S-AGA).

La comparaison des gains attendus de la S-ARA ou de la S-AGA par rapport à ceux d'une sélection pratiquée en culture pure a montré une supériorité des méthodes tenant compte de l'aptitude à l'association lorsque la sélection porte sur des traits soumis à des effets de compétition ou de coopération entre plantes, génétiquement déterminés. Ainsi, il est préférable d'utiliser des méthodes conçues pour l'amélioration des performances en mélange afin de valoriser les associations entre espèces.

Les recherches sur les mécanismes favorables à la diversité et les traits à sélectionner pour des cultures diversifiées valorisent la littérature de l'écologie des communautés. Litrico et Violle (2015) proposent de relier certains traits majeurs à la dynamique de 
croissance, à la phénologie et à l'architecture aérienne, mais il existe encore peu de preuves expérimentales de ces relations. Des travaux prennent néanmoins ce parti pris (Prieto et al., 2015 ; Meilhac et al., in press).

L'effet de la diversité génétique sur le fonctionnement des communautés végétales constitue un front de recherche. Peu d'études se sont penchées sur le sujet. Certains travaux récents (Zuppinger-Dingley et al., 2014 ; Prieto et al., 2015 ; Schöb et al., 2018) montrent des effets de la diversité génétique sur le fonctionnement des communautés, en particulier sur les prairies (Meilhac et al., 2019). Ces travaux s'inscrivent dans une démarche d'analyse des relations entre la diversité génétique, le fonctionnement des communautés et la fourniture de services écosystémiques dans les agroécosystèmes.

Des projets encore en cours ont pour objectif d'exploiter les avantages des mélanges d'espèces, comme le projet ReMIX (Redesigning European Cropping Systems Based on Species Mixtures), qui porte essentiellement sur les céréales et les légumineuses à graines pour concevoir des systèmes de culture agroécologiques plus diversifiés et résilients face aux aléas (économiques ou climatiques), moins dépendants des intrants (engrais chimiques, pesticides...). Fondé sur une approche pluridisciplinaire et multi-acteurs, il a vocation à produire de nouvelles connaissances transférables en agriculture conventionnelle et en agriculture biologique. Il aborde les questions pratiques pour élaborer des solutions adaptées à la production des principales cultures de céréales et dans les différentes conditions pédoclimatiques de l'Europe. Le projet intègre à la fois l'identification des besoins des utilisateurs finaux, la coconception des expérimentations au champ et dans les exploitations agricoles et l'évaluation de nouvelles variétés et de pratiques. La sélection participative est un autre type d'avancée scientifique. Elle s'est développée sur les céréales. Elle modifie l'organisation de la sélection, prend en compte la valorisation des ressources génétiques et joue sur le choix des critères de sélection. La France est particulièrement engagée dans cette voie.

\section{- Les animaux}

En sélection animale, l'objectif de concilier progrès génétique et maintien de la diversité intrarace est un sujet ancien. L'objectif était alors soit de maximiser le progrès génétique à un niveau de consanguinité fixé, soit de ne pas dépasser un certain degré de consanguinité à un niveau de progrès génétique fixé. Ces travaux reposaient uniquement sur l'utilisation des relations de parenté. L'application de ces méthodes a été bouleversée par le séquençage des génomes et par l'accès à un marquage dense du génome, qui ont permis de caractériser et de piloter la diversité à un niveau bien plus fin qu'auparavant en analysant la variation de diversité en fonction de la région du génome. Cette évolution a culminé avec la mise en œuvre de la sélection génomique, dont la publication fondatrice date de 2001 (Meuwissen et al., 2001). La généralisation d'outils de génotypage peu coûteux et l'amélioration des méthodes de calcul ont permis leur application en routine chez les bovins depuis 2009. Des méthodes ont été récemment développées 
pour estimer la part génétique des interactions sociales afin de traiter de questions relatives au bien-être animal. La génomique vient ainsi améliorer la prédiction des interactions entre animaux. L'influence d'un animal sur les performances des autres animaux au sein d'un même groupe peut ainsi être modélisée et exploitée pour diminuer les comportements de cannibalisme chez la poule pondeuse (Alemu et al., 2016). Ces méthodes peuvent être généralisées pour d'autres caractères d'intérêt qui restent à identifier dans des systèmes agroécologiques.

La génétique animale repose actuellement soit sur la diversité de l'offre des reproducteurs pour l'adaptation aux besoins plus ou moins locaux (cas des ruminants), soit sur une plus grande combinatoire de lignées pour le croisement (porc ou volailles). Mais le niveau de diversité intratroupeau n'est pas un objectif en soi, alors que Blanc et al. (2013) ont montré par la modélisation que les troupeaux diversifiés (diversité intrarace dans le troupeau) sont plus stables, rejoignant ainsi des constats faits en génétique végétale avec les mélanges variétaux et les mélanges d'espèces.

\section{Les couverts forestiers}

Dans le cas des forêts, deux voies sont favorisées pour valoriser la diversité génétique : - celle de l'enrichissement génétique des peuplements, avec l'objectif de maintenir la diversité (in situ) pour les climats de demain et de favoriser la création de la diversité en mélangeant des pools génétiques qui se croiseront (provenances allochtones, variétés améliorées de la même espèce, voire d'espèces apparentées avec lesquelles le peuplement en place peut s'hybrider) ;

- celle de l'utilisation d'espèces exotiques, voie qui n'est pas nouvelle et souvent présente dans des systèmes de plantation-replantation (cas du pin douglas). Les espèces introduites peuvent se régénérer naturellement (cas du cèdre). Ce type de peuplement, si sa diversité génétique est suffisante, présente l'avantage de limiter la consanguinité dans les premières générations.

Ces deux stratégies, combinant plantation et régénération naturelle, enrichissement génétique et espèces introduites en régénération, comportent des risques et des avantages qu'il faut évaluer, des incertitudes qu'il faut gérer.

\section{Quelques exemples}

\section{Le projet Praise}

Le projet Praise (soutenu par un financement de l'ANR) est focalisé sur la prairie temporaire semée plurispécifique, dont la diversité intra et interspécifique est une composante jusqu'ici peu exploitée. L'objectif du projet était de poser les bases génétiques et écologiques de l'amélioration des espèces destinées à une utilisation en mélange, notamment 
pour faire face aux aléas climatiques. Il s'agissait d’identifier les conditions génétiques et écologiques qui favorisent une production importante et stable des prairies multispécifiques au cours du temps, et de fournir les bases théoriques d'un schéma de sélection innovant des espèces fourragères prairiales dans la perspective d'une utilisation en mélanges plurispécifiques. Plusieurs disciplines (génétique quantitative, écophysiologie, écologie fonctionnelle des communautés, génétique des populations), plusieurs approches (combinant expérimentations et modélisation) ont été mises en œuvre pour analyser et pour comprendre l'effet de la distribution des caractères fonctionnels. L'utilisation de modèles a permis d'analyser l'effet de la variabilité génétique intraspécifique dans la production des communautés prairiales. Alors que la diversité spécifique améliore la production de biomasse cumulée en régime hydrique limitant, la diversité génétique influence positivement la stabilité de la production des communautés prairiales semées, dans les conditions testées. Par ailleurs, l'analyse de la variabilité de la morphogenèse aérienne et racinaire d'un large panel d'espèces, choisies pour leur importance agronomique et leurs traits morphologiques contrastés, a permis de vérifier la robustesse des hypothèses sur lesquelles est fondée la morphogenèse dans les modèles de production actuellement développés en incluant la diversité génétique des espèces (Louarn et Faverjon, 2018).

Après plusieurs années de recherche et de sélection spécifique dédiées aux mélanges fourragers multi-espèces, l'entreprise Jouffray-Drillaud, filiale de Terrena et partenaire du projet Praise, a lancé « M les Mélanges », gamme innovante de mélanges fourragers multi-espèces dont le comportement de chacune des variétés a été testé en situation de mélange, afin de fournir à l'éleveur les données des performances techniques du champ et à l'auge, et ce, sur la durée. La mise au point de ces compositions est le fruit des essais menés par JouffrayDrillaud pendant quatre ans, en collaboration avec les équipes de l'INRA de Lusignan. Les compositions fourragères n'avaient jusque-là résulté que de l'association de variétés spécifiquement sélectionnées, testées et évaluées pour leur performance en association, pour, finalement, plus de productivité, un fourrage plus équilibré et une haute valeur alimentaire. Afin de mettre au point ces compositions d'un nouveau genre, l'aptitude à la compétition entre espèces, la vitesse d'implantation et la résilience comme faculté de récupération de la plante suite à un stress ont été, pour la première fois, prises en compte.

\section{Le projet Wheatamix}

Le projet Wheatamix (soutenu par un financement de l'ANR) s'est quant à lui attaché à mieux évaluer le rôle possible des associations variétales dans un champ pour renforcer la multifonctionnalité et la résilience des systèmes agricoles dans le contexte du changement global, et s'est focalisé sur la production de blé dans le Bassin parisien. Une approche pluridisciplinaire des chercheurs (généticiens, agronomes, écophysiologistes, écologistes, économistes, chercheurs en sciences de gestion) et des acteurs de la filière (chambre d'agriculture, agriculteurs) a là aussi été initiée. Le projet a contribué à mieux comprendre les interactions entre les variétés, entre celles-ci et le milieu, pour obtenir des associations performantes, que ce soit en matière de rendement et de qualité, de 
services écosystémiques ou encore de débouchés pour la production. Il visait notamment à établir de façon participative des règles d'assemblage des variétés et à évaluer leur pertinence dans différents contextes de production (Barot et al., 2017).

\section{Le projet Riz éternel}

Ce projet (financé dans le cadre du métaprogramme Inra $\mathrm{SMaCH}^{50}$ ) a étudié la gestion durable des résistances variétales du riz dans les rizières chinoises de Yuanyang inscrites au patrimoine mondial de l'Unesco (sud du Yunnan). Il combine des approches de génétique, de génomique, d'analyse des réseaux sociaux et de modélisation. Dans cette région où les agriculteurs n'utilisent pas de fongicide, le riz est étonnamment préservé des épidémies : si la présence de Magnaporthe oryzae y est avérée, ces rizières ne sont que très peu affectées par le champignon. Or deux types de variétés de riz sont co-cultivés : du riz ordinaire (indica) et du riz gluant utilisé pendant les fêtes (japonica). Les travaux ont montré que les variétés japonica présentent une forte immunité basale et peu de gènes de résistance, tandis qu'à l'inverse les variétés indica possèdent une faible immunité basale et beaucoup de gènes de résistance. Ces deux types de variétés de riz ayant des systèmes immunitaires si différents ont conduit à l'existence de deux populations spécialisées de champignons capables d'infecter des variétés spécifiques de riz. Ces deux types d'agents pathogènes ultra-spécialisés sont donc incapables de se disperser dans un paysage hétérogène.

Cette étude fait le lien entre deux des articles les plus cités en biologie des plantes ces quinze dernières années. L'un porte sur un modèle de l'immunité des plantes (modèle zigzag) qui prédit qu'un mélange approprié entre immunité basale et gènes de résistance pourrait accroître la durabilité des résistances. Le second décrit un exemple, chez le riz, de succès d'utilisation de la diversité des plantes comme générateur de durabilité de la résistance à la pyriculariose. Ces résultats montrent que le déploiement de systèmes immunitaires diversifiés permet de proposer des agrosystèmes présentant une protection des plantes durable, et ouvrent ainsi des perspectives pour d'autres plantes de grande culture, notamment pour le blé.

\section{Exemples en production animale}

Dans le cas des animaux, les exemples sont de trois types.

1. Mobiliser la diversité génétique pour élaborer des systèmes de production plus résilients qui peuvent être qualifiés d'agroécologiques a posteriori. Dans l'expérimentation ovine entreprise au domaine expérimental de La Fage (Saint-Jean-et-Saint-Paul, causse du Larzac à La Fage) depuis les années 1970, une recherche pluridisciplinaire associant la génétique, la zootechnie, la physiologie, l'éthologie et l'écologie a abouti à un système de production ovin allaitant en plein air intégral montrant un haut niveau de performances

50. Sustainable Management of Crop Health. 
dans un milieu à fortes contraintes pédoclimatiques. La comparaison des performances zootechniques de plusieurs races et génotypes (Romanov, Lacaune, F1) a permis la constitution d'un troupeau de brebis Romane sur les parcours. Cette race composite (mélange des races complémentaires Romanov et Berrichon du Cher) présente de bonnes aptitudes comportementales et physiologiques pour s'adapter aux conditions environnementales contraignantes. Le milieu a aussi été étudié au travers d'un suivi à long terme des communautés végétales (biomasse, diversité) et des caractéristiques des espèces. La mise en place de la fertilisation modérée par l'homme s'est accompagnée d'un changement drastique de la végétation au profit d'espèces annuelles, avec une augmentation de la biomasse produite et une augmentation de la qualité des feuilles par rapport au milieu pâturé uniquement. D'autres projets de ce type se sont mis en place en climat chaud dans un contexte expérimental et partenarial, comme en Guadeloupe, où les populations créoles, issues de métissage, présentent une remarquable adaptation à un milieu difficile, ou encore dans un contexte d'aide au développement, avec le programme d'une ONG pour la sélection de la vache laitière en Inde, financée par la Fondation Bill et Melinda Gates. Dans ce cas, la méthode est participative et l'usage du croisement est raisonné pour le maintien des capacités d'adaptation des vaches laitières à un climat chaud.

2. Associer la production sous signe de qualité au maintien de la diversité génétique, comme dans le cas de races ovines locales (Nozières-Petit et Lauvie, 2018). Le cas du poulet de Bresse a été pionnier, mais son objectif est d'abord la qualité du produit avant la valorisation des régulations biologiques.

3. Identifier des marqueurs génétiques liés à des caractères favorables à l'essor de systèmes agroécologiques. Ces marqueurs sont intégrés aux programmes de sélection en cours, sans être encore mobilisés pour la mise en place de systèmes agroécologiques. Il s'agit notamment de l'efficacité alimentaire (substitution alimentaire, diminution des intrants et de la pollution ; projets chez les ruminants, les volailles, les poissons) et de la résistance aux maladies (addition de critères de résistance aux maladies dans les programmes de sélection, diminution des intrants). En Guadeloupe, des plans expérimentaux originaux couplés aux approches de génomique ont été mis en place pour étudier l'adaptation au chaud chez le porc et la résistance aux nématodes chez la chèvre.

\section{Les questions de recherche}

DEUX FRONTS de recherche majeurs sont identifiés.

\section{Comprendre et estimer la part génétique de la variation due aux interactions au sein des peuplements complexes}

L'objectif est de développer des méthodes de sélection pilotant ou tirant parti de la variabilité génétique des interactions dans les espèces. À ce front de science sur les 
interactions entre plantes ou entre animaux, l'approche basée sur les traits apporte un aspect fonctionnel et laisse la possibilité d’identifier des critères de sélection chez les plantes et les animaux.

\section{I Étudier les relations entre la diversité génétique et les services écosystémiques rendus par les systèmes de culture et/ou d'élevage}

Les questions concernent : la marge de progrès que peut conférer le levier génétique à l'amélioration des services écosystémiques, la gamme de diversité efficace et à son déploiement dans le cadre d'environnements hétérogènes où l'interaction génétiqueenvironnement devient centrale, et l'identification des traits des plantes et des animaux, qui doivent être diversifiés ou qui ne doivent pas l'être pour optimiser les services écosystémiques attendus en maintenant les niveaux de production.

\section{IVerrous méthodologiques associés}

Il s'agit d'étudier la génétique des interactions $\mathrm{G} \times \mathrm{M} \times \mathrm{E} \times \mathrm{C} \times \mathrm{P}$ :

$\mathrm{G}$ : variabilité génétique entre individus (plantes, animaux, arbres) et entre populations ; M : variabilité de l'environnement microbien : microbiote intestinal, microbiote de la feuille, des racines, du sol ${ }^{51}$;

E : variabilité du milieu physique (climat, ressources en eau, système hydrologique, distribution des sols...) en lien avec le fonctionnement de l'agroécosystème ;

$C$ : conduite de l'exploitation, du système de production et du système de culture, avec notamment la question de la modélisation des choix de l'agriculteur ;

$P$ : variabilité de la matière première issue d'exploitations agroécologiques, qui remet en cause le paradigme selon lequel la transformation peut tout faire en gommant la diversité initiale.

Il s'agit d'intégrer différents niveaux de diversité dans les démarches de conception de systèmes de culture et d'élevage : ces systèmes devront être évalués par des approches multicritères afin de relier les impacts aux objectifs de sélection. Cela suppose de proposer des stratégies de sélection pour des populations en mélanges de races ou de lignées ou pour une utilisation en croisement, afin de mieux répondre aux besoins des systèmes à faibles niveaux d'intrants. Ces stratégies doivent être coconstruites avec les acteurs : quels modes d'organisation des acteurs pour quelle méthode de sélection ? Comment définir une ressource génétique adaptée ? Ces sujets demandent une recherche participative.

51. La sélection végétale parle de phénotype augmenté ; la sélection animale identifie le microbiote comme une nouvelle composante de la prédiction des performances; la notion d'holobionte (individu + microbiome) se développe. 
Il s'agit de revoir les processus de transformation : utiliser la diversité pour équilibrer le produit final, au lieu de gommer la variabilité par un processus de transformation qui altère le matériau de base. Autrement dit, la variabilité initiale ne pourrait-elle pas être un atout ? Les relations entre les caractéristiques génétiques initiales et la qualité du produit final doivent être mieux établies. L'élaboration du produit étant la dernière étape avant le consommateur, celle-ci est déterminante de l'acceptabilité de systèmes valorisant la diversité génétique.

\section{Expérimentation et dispositifs de recherche}

Les approches méthodologiques en génétique animale ou végétale vis-à-vis de la valorisation de la diversité génétique pour l'agroécologie convergent bien entre elles. La convergence est en revanche plus difficile avec les approches « systèmes ». Même si certains projets récents proposent de relancer l'utilisation du croisement chez les ruminants, le plus souvent la diversité génétique n'est pas considérée comme un paramètre prioritaire dans les expérimentations systèmes, alors que l'agroécologie se caractérise par son approche système et que la diversité génétique est un levier à considérer et à optimiser. La même situation se rencontre dans les travaux d'économie qui n’intègrent pas la dimension de la variabilité génétique dans les modèles développés, notamment parce que les données manquent. Des collaborations entres sciences économiques et génétique animale ou végétale donneront accès à des données, ou du moins à des connaissances pour les simuler dans un modèle.

Traiter ces questions de recherche suppose des dispositifs particuliers pour l'acquisition de données. La modélisation des interactions suppose de disposer de méthodes et de coupler les modèles de génétique et d'écophysiologie, mais aussi de collecter un grand nombre de données en intégrant différents types de données. Les dispositifs expérimentaux actuels n'offrent pas assez de combinaisons pour comprendre les mécanismes de l'adaptation des génotypes à un système agroécologique. La sélection dans un système changeant (environnements et espèces d'intérêt) doit mieux impliquer l'aval, associer les agriculteurs et les éleveurs sur un mode participatif pour accroître la diversité des situations étudiées. Des réseaux de fermes et d'autres dispositifs coconstruits avec des acteurs sont nécessaires pour l'étude de l'adaptation. Ces deux niveaux d'acquisition de données, la collecte de données en milieu expérimental bien connu (phénotypage fin) et la collecte de données à la ferme, doivent être combinés. Modéliser les interactions au sein des peuplements est utile, avec par exemple des modèles centrés sur l'individu qui représentent les interactions d'individu à individu, qui intègrent les pressions de sélection locales dues à l'individu voisin et à ses effets sur les paramètres abiotiques. Leur échelle d'application est en principe la parcelle ou le troupeau, mais ce niveau d'intégration est encore peu présent, notamment en génétique animale, qui utilise plutôt des modèles à l'échelle de la population en sélection.

Les approches fonctionnelles, en conditions expérimentales, permettent, de façon complémentaire, de déterminer les traits majeurs de la réponse des individus aux 
conditions du milieu (sol, climat, nutriments) et de proposer de nouveaux critères de sélection. Par exemple, une façon d'améliorer la valorisation des protocoles de sélection animale serait de prévoir le génotypage systématique de tous les animaux inclus dans des expérimentations systèmes, avec un outil de génotypage commun, afin de regrouper les données et d'identifier des marqueurs génétiques associés à une plus grande robustesse des troupeaux dans différentes conditions de milieu.

Les expérimentations systèmes contribuent à la recherche d'innovation. Cependant, la conception de systèmes de culture incluant le levier génétique est encore aujourd'hui rare et se révèle compliquée en raison notamment, mais pas uniquement, de la difficulté d'articuler les niveaux d'organisation du génome à la communauté et de faire le lien entre l'approche du système et des travaux ciblés sur une discipline.

En conclusion, les méthodes de sélection en génétique végétale comme en génétique animale bénéficient d'outils permettant un suivi fin de la diversité génétique qui doivent être mis à profit pour la transition agroécologique. Le changement majeur impulsé par l'agroécologie est non plus de chercher l'individu idéal, mais le groupe idéal pour un contexte de production donné. Cela conduit à une reconception des objectifs de sélection et des schémas de sélection, au-delà de l'identification de nouveaux critères de sélection. Elle doit se faire conjointement avec l'évolution des systèmes de culture ou d'élevage pour mieux valoriser la diversité génétique. Il reste encore à mieux intégrer la question du choix du génotype aux différentes échelles de l'exploitation à la filière.

\section{Références bibliographiques}

Alemu S.W., Calus M.P.L., Muir W.M., Peeters K., Vereijken A., Bijma P., 2016. Genomic prediction of survival time in a population of brown laying hens showing cannibalistic behaviour. Gen. Select. Evol., 48, 68.

Barot S., Allard V., Cantarel A., Enjalbert J., Gauffreteau A., Goldringer I., Lata J.-C., Le RouxX., Niboyet A., Porcher E., 2017. Designing mixtures of varieties for multifunctional agriculture with the help of ecology. A review. Agron. Sustain. Dev., 37, 20.

Blanc F., Ollion E., Puillet L., Delaby L., Ingrand S., Tichit M., Friggens N.C., 2013. Évaluation quantitative de la robustesse des animaux et du troupeau : quels principes retenir ? Renc. Rech. Ruminants, 20, 265-272.

Litrico I., Violle C., 2015. Diversity in plant breeding: a new conceptual framework. Trends Plant Sci., 20, 604-613.

Louarn G., Faverjon L., 2018. A generic individual-based model to simulate morphogenesis, C-N acquisition and population dynamics in contrasting forage legumes. Ann. Bot., 121, 875-896.

Meilhac J., Durand J.L., Beguier V., Litrico I., 2019. Increasing the benefits of species diversity in multispecies temporary grasslands by increasing within-species diversity. Ann. Bot., 123, 891-900. DOI: $10.1093 /$ aob/mcy227.

Meilhac J., Maire V., Deschamps L., Flajoulot S., Litrico I., (in press). Species differentiation in grassland canopy is mediated by selection and plasticity. Nature Plants. 
Meuwissen T.H., Hayes B.J., Goddard M.E., 2001. Prediction of total genetic value using genome-wide dense marker maps. Genetics, 157, 1819-1829.

Nozières-Petit M.-O., Lauvie A., 2018. Diversité des contributions des systèmes d'élevage de races locales. Les points de vue des éleveurs de trois races ovines méditerranéennes. Cahiers Agricultures, 27,65003 .

Phocas F., Belloc C., Bidanel J., Delaby L., Dourmad J.Y., Dumont B., Ezanno P., Fortun-Lamothe L., Foucras G., Gonzales-Garcia E., Hazard D., Larzul C., Lubac S., Mignon-Grasteau S., Moreno C.R., Tixier-Boichard M., Brochard M., 2016a. Review: towards the agroecological management of ruminants, pigs and poultry through the development of sustainable breeding programmes. I. Selection goals and criteria. Animal, 10, 1749-1759.

Phocas F., Belloc C., Bidanel J., Delaby L., Dourmad J.Y., Dumont B., Ezanno P., Fortun-Lamothe L., Foucras G., Gonzales-Garcia E., Hazard D., Larzul C., Lubac S., Mignon-Grasteau S., Moreno C.R., Tixier-Boichard M., Brochard M., 2016b. Review: towards the agroecological management of ruminants, pigs and poultry through the development of sustainable breeding programmes. II. Breeding strategies. Animal, 10, 1766-1776.

Prieto I., Violle C., Barre P., Durand J.-L., Ghesquiere M., Litrico I., 2015. Complementary effects of species and genetic diversity on productivity and stability of sown grasslands. Nature Plants, 1, 15033.

Sampoux J.-P., Giraud H., Litrico I., (in press). Which recurrent selection scheme to improve mixtures of plant species? Theoretical expectations. 3G: Genes, Genomes, Genetics.

Schöb C., Brooker RW., Zuppinger-Dingley D., 2018. Evolution of facilitation requires diverse communities. Nature Ecology and Evolution, 2, 1381-1385

Zuppinger-Dingley D., Schmid B., Petermann J.S., Varuna Y., de Deyn G.B., Flynn D.F.B., 2014. Selection for niche differentiation in plant communities increases biodiversity effects. Nature, 515, 108-111. 



\section{Modéliser}

\section{les interactions du vivant,}

en lien avec les milieux et le contexte socio-économique

LA MODÉLISATION EN AGROÉCOLOGIE EST UN DÉFI, d'une part parce que l'agroécologie est naissante en France et en évolution dans le monde, et donc que les connaissances et les corpus de données pour l'ancrer sont en émergence, d'autre part parce qu'elle implique par nature la modélisation de systèmes complexes dans un environnement incertain. Les systèmes représentés sont des systèmes dynamiques, et les interactions entre les organismes vivants y sont nombreuses et variées. Pour autant, cette modélisation est attendue pour orienter et accompagner la transition des systèmes agricoles. Si la prévision par la modélisation est encore difficile, celle-ci peut aider à comprendre la dynamique des agroécosystèmes, à orienter des pistes d'action, à identifier des points d'attention et des impasses, à établir des systèmes d'avertissement pour anticiper et aider au pilotage des agroécosystèmes, à estimer les ordres de grandeur des risques et des gains induits par la transition agroécologique.

L'agroécologie a pour ambition d'exploiter des processus biologiques riches et variés à des fins de production agricole et de durabilité. Les travaux de modélisation conçus en appui à cette ambition ont pour objectif de représenter et de prédire ces processus biologiques et leurs interactions dans les agroécosystèmes, éventuellement couplés à des dynamiques des milieux, ou à des dynamiques sociales ou économiques. Ces modèles contribuent à représenter, partiellement ou totalement, la cascade des connaissances sur les systèmes et pratiques agricoles, les paysages ruraux, la biodiversité, les fonctions et services écosystémiques des agroécosystèmes.

À ces modèles représentant explicitement la biodiversité (process driven) s'adjoignent des approches statistiques fondées sur les données (data driven) qui établissent des relations entre les composantes de la biodiversité et leurs fonctions.

D’autres modèles, qui n'avaient initialement pas été conçus pour l'agroécologie, constituent aussi des ressources par exemple quand les méthodes mises en œuvre ont une vocation générique utile pour l'agroécologie, ou quand les interactions entre organismes ne sont pas encore explicitées, mais pourraient l'être, ou commencent à l'être, sous la forme de fonctions empiriques. C'est le cas de l'activité des microorganismes du sol et de leur rôle dans la disponibilité en nutriments et dans les cycles biogéochimiques : ils commencent à être pris explicitement en compte, en s'appuyant sur de grands groupes fonctionnels. 


\section{Des avancées scientifiques récentes}

Le domaine de la modélisation en agró́cologie est en plein essor. À titre d'exemple, 107 modèles ont été recensés à l'Inra en 2018 (Monod et al., 2018). Ces modèles ont contribué à environ 400 publications, en grande partie publiées très récemment. Ils correspondent, pour certains, à des familles de modèles, pour d'autres, à un modèle unique. La communauté scientifique concernée commence à se structurer en quelques réseaux de collaboration. Une enquête réalisée auprès des concepteurs de cette centaine de modèles permet d'analyser ce que peuvent faire les modèles ainsi que leurs limites dans le champ de l'agroécologie.

\section{De nombreux domaines et types de processus écologiques représentés}

Les modèles en agroécologie couvrent de nombreux domaines :

- les interactions entre génétique et environnement ;

- les relations entre plantes, bioagresseurs et l'usage des pesticides ;

- les cultures associées et l'accès aux ressources (eau, nutriments) ;

- la santé animale, la gestion antibiotique ou des populations de vecteurs (tiques...) en lien avec la faune sauvage ;

- l'exploitation agricole, son atelier d'élevage et la gestion de la ressource végétale, ses troupeaux avec la prise en compte des relations intra et inter-troupeaux ;

- les politiques de conservation (stratégie de conservation, relations entre espèces).

Plusieurs catégories d'interactions ou de fonctions écologiques y sont étudiées : parasitisme, prédation, compétition, facilitation, décomposition de la matière organique. Des modèles relatifs à la distribution spatio-temporelle de populations ou de particules biotiques sont identifiés. Différentes échelles sont visées : intra-hôte, population, territoire, etc. Quelques exemples significatifs peuvent être cités.

1. Un cadre générique de simulation en épidémiologie (EMuLSion) a été développé (Picault et al., 2017). Les processus d'infection et de cycle de vie des animaux sont représentés sous forme de « machines à états » qui permettent de décrire des systèmes séquentiels dont l'évolution est plus complexe que de simples compteurs ou registres.

2. La biodiversité devient explicitement modélisée pour des phases clés des cycles biogéochimiques, avec la représentation de groupes fonctionnels microbiens et de leur rôle dans des modèles de décomposition de la matière organique des sols (modèles GDM, CANTIS, EeZY ; lqbal et al., 2014 ; Moorhead et al., 2012, 2014).

3. Un environnement de modélisation et de simulation de dynamiques de populations sur paysages réalistes (Esomed : Environnement de simulation et d'optimisation de modèles d'équations aux dérivées partielles) est désormais mis en place (Roques et Bonnefon, 2016). Cet environnement permet de coupler des dynamiques sur des parcellaires à deux dimensions et sur des éléments à une dimension du paysage (haies, routes...). L'environnement intègre un générateur stochastique de parcellaires et d'usages des sols. 


\section{Des systèmes agricoles aux services des agroécosystèmes en passant par la biodiversité}

La modélisation en agroécologie commence à parcourir réellement la cascade allant des systèmes agricoles, des paysages ruraux, des pratiques agricoles à la biodiversité et aux fonctions et services des agroécosystèmes. La biodiversité est désormais largement représentée dans les modèles. Ces modèles répondent quasiment tous à un besoin de compréhension, certains s'inscrivant dans une écologie théorique, d'autres allant jusqu'à traiter des fonctions de la biodiversité, voire des services écosystémiques qui en résultent. Les modèles ayant vocation à répondre à des enjeux finalisés sont désormais prépondérants. Les relations avec les milieux ou avec les acteurs sont souvent modélisées par un compartiment indépendant, couplé avec le compartiment biologique. Mais ces couplages, notamment ceux représentant les dynamiques d'acteurs, sont encore peu nombreux. L'intégration de processus dynamiques s'opérant à différentes échelles reste un défi pour la modélisation en agroécologie, y compris sur le plan méthodologique.

\section{Un besoin d'ancrage sur des données de terrain}

Les modèles doivent davantage s'ancrer sur des données de terrain pour aborder des enjeux finalisés. La plupart des modèles ont vocation à produire et à tester des scénarios de gestion des agroécosystèmes, mais le nombre d'approches allant jusqu'aux prévisions, au sens d'évolution de court terme pour une adaptation tactique, est encore réduit. Les modèles intègrent plus souvent une composante temporelle que spatiale.

\section{Des débuts de démarches probabilistes}

Des démarches probabilistes voient le jour. La plupart des modèles en agroécologie s'appuient sur un formalisme mathématique et intègrent une composante probabiliste, ce qui permet la prise en compte d'incertitudes, ou une simplification du modèle, en évitant par exemple la modélisation de mécanismes à faible effet. L'intérêt de ces démarches probabilistes est surtout de fournir des prévisions sous forme d'enveloppes de confiance, plutôt que des trajectoires uniques.

\section{Quelques exemples}

\section{Des réseaux d'animation scientifique interdisciplinaires sur la modélisation en agroécologie}

Le réseau ModStatSAP ${ }^{52}$, créé en 2011, a pour objectif de fédérer les modélisateurs et les statisticiens travaillant sur les dynamiques des systèmes hôtes-pathogènes ou

52. Modélisation et statistique en santé des animaux et des plantes. 
hôtes-bioagresseurs. Depuis sept ans, le réseau participe à les fédérer, notamment en maintenant un site web, une liste de diffusion et un flux Twitter, en organisant des réunions annuelles et des ateliers de travail. Les séminaires annuels et les ateliers du réseau illustrent comment les modèles contribuent à la connaissance des processus épidémiques et démo-génétiques (c'est-à-dire relatifs aux aspects démographiques et évolutifs) à l'échelle des populations ou du paysage, et à la gestion des systèmes agroécologiques à travers, par exemple, l'optimisation du déploiement des résistances, des usages des sols ou encore de la surveillance. L'article de Parisey et al. (2016) (figure 5) porte sur l'influence de l'agencement spatial de paysages agricoles sur la présence d'auxiliaires de culture, et correspond à une concrétisation typique de ces rencontres.

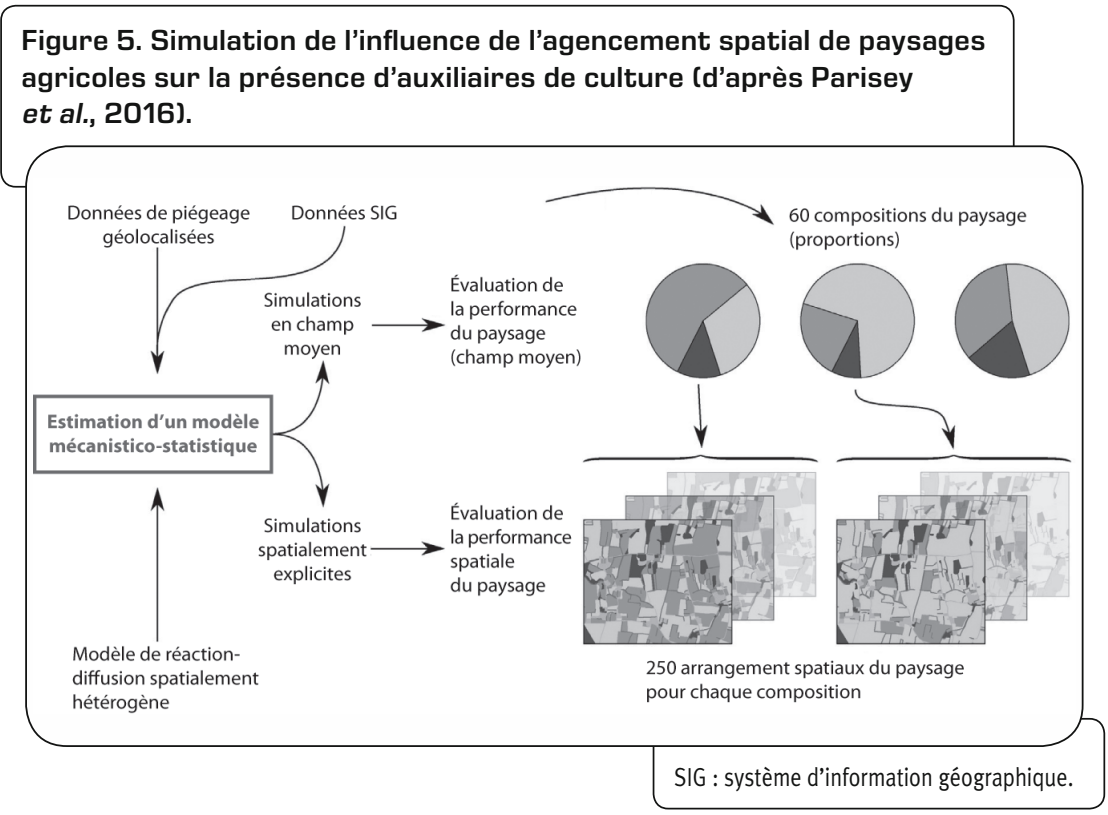

D’autres réseaux se sont aussi créés. Le réseau Payote s'intéresse à la modélisation des paysages agricoles et à l'étude de leur impact sur des phénomènes de propagation et de dynamique de population. Le réseau EpiArch s'intéresse au rôle de l'architecture des plantes sur les processus épidémiques.

\section{Modèles génériques et plateformes}

FLORSYS (pour flore adventice et système de culture ; Colbach et al., 2017) est un modèle qui simule la dynamique pluriannuelle d'une flore adventice plurispécifique et les interactions avec les plantes cultivées au niveau d'un îlot de parcelles et d'habitats semi-naturels, 
en fonction des systèmes de culture et des conditions pédoclimatiques, puis l'impact de la flore adventice sur la production agricole, la biodiversité et l'environnement. Il considère plusieurs types d'interactions (plante-plante, plante-parasite, plante-champignon, plante-prédateur) et de services écosystémiques.

La plateforme Capsis est une plateforme logicielle générique donnant accès à des modèles de croissance (croissance et mortalité des arbres) pour des formations variées, plus ou moins hétérogènes, pures ou en mélanges d'espèces, en zone tempérée, mais également subtropicale et tropicale, et pour divers types de gestion (Dufour-Kowalski et al., 2012). Cette plateforme, préconisée surtout pour la gestion forestière, est aussi utilisée pour les agrosystèmes arborés (vergers...). Elle intègre un ensemble d'équations mathématiques qui relient les caractéristiques dendrométriques des arbres (hauteur, diamètre, volume...) et du peuplement qu'ils constituent, leur nombre, leur âge, et qui traduisent leur évolution selon la compétition qui s'exerce entre eux.

\section{Les questions de recherche}

\section{Déficit de connaissances scientifiques}

Un frein scientifique important auquel doit faire face la communauté de recherche en modélisation pour l'agroécologie est le manque de connaissance des mécanismes d'interaction et leur intégration dans des approches systémiques sur des objets complexes tels que l'exploitation agricole ou le paysage. Les modèles élémentaires, ou « briques de base ", décrivant séparément chaque compartiment du système étudié, peuvent être calibrés sur la base de données ou de connaissances expertes. Ce sont les liens entre ces briques de base qui sont difficiles à établir, par exemple les liens de compétition, de synergie, de prédation, pour ce qui concerne les compartiments biologiques.

Globalement, le nombre d'interactions prises en compte dans les modèles reste faible. Par exemple, les interactions binaires entre plantes et bioagresseurs ou entre plantes sont plus souvent représentées dans les modèles actuels que les interactions multiples. Quelques modèles comme FLoRSYS, qui simule la dynamique pluriannuelle de la flore adventice et les interactions avec les plantes cultivées, et VIRTUAL GRASSLAND, qui simule la dynamique des peuplements prairiaux, font exception. Un autre constat est que peu, voire pas, de travaux portent sur l'interaction entre biodiversité souterraine et aérienne.

\section{Difficultés méthodologiques}

Les sorties des modèles sont souvent extrêmement sensibles aux choix des fonctions d'interaction entre compartiments élémentaires. Cette sensibilité rend hasardeuse l'approche de modélisation des agroécosystèmes par simple intégration de différentes briques de base dont les fonctions d'interaction ne seraient pas clairement identifiées. 
Les approches s'appuyant sur la mise en interaction de briques de base doivent continuer à être développées, en bénéficiant de données plus adaptées, en structurant mieux les communautés de modélisateurs et d'expérimentateurs, en rendant disponibles dès que possible les briques de base validées. Mais, parallèlement, d'autres approches de la complexité doivent être envisagées. Il s'agit notamment des approches stochastiques, qui évitent la modélisation de mécanismes dont les effets seraient faibles, par exemple en les remplaçant par l'ajout d'un terme de "bruit » résumant la somme de ces effets par une fonction aléatoire. Il s'agit également de l'introduction de contraintes dans les modèles, qui permettent de limiter les besoins en données, comme dans les approches mécanistico-statistiques (Dusseux et al., 2015 ; Soubeyrand, 2016 ; figure 5). Ces méthodes combinent un modèle mécaniste (fondé sur des processus et intégrant certaines contraintes), un modèle probabiliste faisant le lien entre processus et données, et une méthode statistique d'estimation. Enfin, il faut explorer de nouvelles pistes, par exemple celle de modèles calibrés à partir de données résultant d'interactions complexes, comme l'assimilation de données issues de la télédétection.

\section{Rareté des données sur des systèmes en transition}

La rareté des données sur des systèmes en transition ou conduits en agroécologie est un écueil fondamental : peu d'expérimentations sont faites et peu de données sont acquises, alors que le besoin est énorme pour approcher le grand nombre d'interactions mises en jeu dans ces systèmes. La modélisation peine à s'appuyer sur un corpus de données variées et de moyen terme, intégrant de nombreuses conditions pédoclimatiques et agronomiques. Une priorité est donc de mieux utiliser et valoriser les dispositifs d'observation ou d'expérimentation, de les faire évoluer afin qu'ils s'engagent davantage dans le champ de l'agroécologie. Les zones ateliers sont en particulier utiles aux échelles du paysage et du territoire. Il faut aussi s'appuyer sur des informations issues de la société. Des expériences en agroécologie mises en place par les Civam, les chambres d'agriculture, les réseaux d'agriculture biologique peuvent apporter des connaissances expertes et des données sur une grande diversité de systèmes. Il existe de nombreuses données sur les systèmes conduits en agriculture biologique dans le monde, comme en témoignent les méta-analyses sur les performances comparées entre les systèmes en agriculture biologique et les autres systèmes. La question de leur accessibilité se pose. Des initiatives en sciences participatives peuvent également contribuer à enrichir les contextes d'observation.

\section{Nature aléatoire et incertaine de certains processus}

La meilleure compréhension et l'intégration dans la modélisation de la nature aléatoire, incertaine, parfois chaotique de certains processus, en particulier de ceux qui sont en interaction avec des processus abiotiques en lien notamment avec le climat, sont des enjeux majeurs. Des méthodes d'analyse des risques dans les systèmes en agroécologie 
ou en transition vers l'agroécologie doivent être développées. La notion de risque est importante à intégrer dans des démarches de modélisation prédictive ; elle va de pair avec la notion de gestion adaptative, gestion qui s'adapte au fur et à mesure de l'évaluation des états, des contraintes et des risques. La notion de risque s'inspire souvent de la littérature économique et mathématique, par exemple de la représentation mathématique de la théorie de la viabilité. La communauté scientifique doit s’impliquer dans des échanges sur les concepts et la représentation de la notion de risque dans les systèmes agroécologiques. La notion de résilience, qui traduit la capacité d'un système à retourner vers un état d'équilibre après des chocs climatiques ou des maladies, devrait, elle aussi, être plus mobilisée. Il s'agit d'instruire la question de la capacité des systèmes agroécologiques à acquérir une certaine robustesse et une résilience vis-à-vis de la variabilité climatique, en opposition à une adaptation à un optimum sur les meilleures années climatiques.

\section{Autres dimensions de la modélisation à explorer}

Certains aspects de la modélisation pour l'agroécologie sont encore insuffisamment développés, ou en émergence.

\section{Les dimensions spatio-temporelles}

L'agroécologie questionne la dimension temporelle plus encore que dans les systèmes conventionnels, du fait de l'importance des interactions entre processus qui s'enchaînent dans le temps en lien avec les conditions biotiques et abiotiques (rotation, arrière effet, variabilité climatique...). Pour cela, revisiter les données de long terme est nécessaire, en prenant en compte l'évolution du contexte d'acquisition, qui peut biaiser l'analyse, et en s'appuyant sur des approches statistiques de type patternoriented modelling, qui permettent d'identifier les pas de temps caractéristiques des processus, et les données importantes à acquérir. Il est tout autant nécessaire de renforcer les modèles développés avec des objectifs de court terme. Certains de ces modèles ont été élaborés avec des objectifs de prévision ou de comparaison de scénarios, mais il y a relativement peu de confrontation au réel et au court terme. La scénarisation, c'est-à-dire la construction d'une histoire pertinente pour orienter l'action, traduite pour être testée par simulation avec une question de type « que se passe-t-il si ? ", fait partie de ce champ. Des modélisations sont en cours, qui comportent des aspects de court terme, mais aussi de long terme : sur le biocontrôle (approches fondamentales, telles que la modélisation démo-génétique des petites populations ou l'étude de la dépendance des insectes vis-à-vis des organismes symbiotiques) ; sur l'écologie du paysage, avec la régulation biotique, l'analyse géomatique, l'épidémiologie, la régulation des flux d'éléments chimiques et biologiques ; sur la symbiose, dont les avantages pour l'agriculture restent encore limités ; sur l'épidémiologie pour des stratégies de maîtrise des maladies. 


\section{Couplage des processus biotiques et abiotiques}

Des modèles couplant des processus biotiques et abiotiques commencent à être développés à l'échelle du paysage, y compris à l'international, et cet effort est poursuivi. Une autre ambition est d'amplifier la prise en compte des composantes biotiques dans les modèles de cycles des nutriments (modèles GDM, EEzy, Cantis), par exemple dans la décomposition de la matière organique des sols. La connaissance de plus en plus avancée des communautés microbiennes et de leurs fonctions offre des opportunités. La rencontre des écologues microbiens et des scientifiques représentant des fonctions par des relations empiriques peut ouvrir de nouveaux fronts de recherche. Les modèles représentant les réseaux trophiques et leur dynamique font également défaut. On manque par ailleurs de modèles basés sur la biodiversité, représentant simultanément différents processus et services écosystémiques et leurs interactions. La biodiversité est représentée explicitement dans un nombre important de modèles, via des traits, ou des guildes écologiques ${ }^{53}$ dans quelques cas, avec des fonctions associées, sans aller cependant jusqu'à la fourniture des services écosystémiques. Les modèles multiservices écosystémiques sont encore peu présents. Des progrès importants sont encore à faire pour représenter la cascade qui relie les pratiques culturales à la biodiversité, puis aux fonctions et aux services écosystémiques. La gestion des invasions biologiques, en lien notamment avec le changement climatique et les changements de systèmes agricoles et forestiers, reste également un défi.

\section{Évaluation multicritère des performances et des services des agroécosystèmes}

Enfin, inclure la dimension de l'agroécologie dans les outils d'évaluation des performances et des services des agroécosystèmes est une demande sociétale forte. L'évaluation multicritère des systèmes agricoles s'appuie sur des plateformes et des outils (plateforme Means $^{54}$ et base de données Agribalys ${ }^{55}$ ) dans lesquels on trouve des démarches d'analyse du cycle de vie, et des débuts de prise en compte des interactions biotiques. Le cadre DEXI (Bohanec et al., 2008) et les outils, comme MASC (Sadok et al., 2009), DEXI-PM (Pelzer et al., 2012) ou DexIFruits ${ }^{56}$, développés pour évaluer la durabilité de systèmes agricoles, y compris agroécologiques, prennent en compte les interactions biotiques, par exemple dans l'évaluation de l'effet d'amendements organiques divers sur la fertilité du sol. Renforcer cette prise en compte de manière à disposer de méthodes d'évaluation multicritères intégrant bien des dimensions biotiques, par le biais d'indicateurs, voire de couplage avec des modèles dynamiques, est nécessaire.

53. Une guilde écologique définit un ensemble d'espèces partageant la même niche écologique.

54. Multicriteria Assessment of Sustainability. Voir https://www6.inra.fr/means.

55. Voir https://www.ademe.fr/expertises/produire-autrement/production-agricole/passer-a-laction/ dossier/levaluation-environnementale-agriculture/loutil-agribalyser.

56. Voir http://wiki.inra.fr/wiki/deximasc/DEXiFruits/. 


\section{Références bibliographiques}

Bohanec A., Messean S., Scatasta F., Angevin B., Griffiths P.H., Krogh M., Znidarsic S., Dzeroski, 2008. A qualitative multi-attribute model for economic and ecological assessment of genetically modified crops. Ecol. Model., 215, 247-261.

Colbach N., Cordeau S., Garrido A., Granger S., Laughlin D., Ricci B., Thomson F., Messéan A., 2017. Landsharing $v s$ landsparing: how to reconcile crop production and biodiversity? A simulation study focusing on weed impacts. Agric. Ecosyst. Environ., 251, 203-217.

Dufour-Kowalski S., Courbaud B., Dreyfus P., Meredieu C., de Coligny F., 2012. Capsis: an open software framework and community for forest growth modelling. Ann. Forest Sci., 69, 221-233.

Dusseux P., Zhao Y.L., Cordier M.O., Corpetti T., Delaby L., Gascuel-Odoux C., Hubert-Moy L., 2015. PaturMata, a model to manage grassland under climate change. Agron. Sust. Dev., 35, 1087-1093.

Iqbal A., Garnier P., Lashermes G., Recous S., 2014. A new equation to simulate the contact between soil and maize residues of different sizes during their decomposition. Biol. Fertil. Soils, 50, 645-655.

Monod H., Gascuel-Odoux C., Lescourret F., Roques L., Bohan D., Costes E., Courtois P., Fabre F., Faverdin P., Franc A., Hoch T., Phocas F., Steyer J.P., Tchamitchian M., 2018. Modèles en agroécologie : état et perspectives à l'Inra. Modélisation des interactions biotiques, en lien avec des dynamiques abiotiques, sociales et économiques : contribution à la représentation de la cascade pratiques agricoles-biodiversité-fonctions et services écosystémiques des agroécosystèmes, document interne, Inra, $51 \mathrm{p}$.

Moorhead D.L., Lashermes G., Sinsabaugh R.L., 2012. A theoretical model of C- and N-acquiring exoenzyme activities, which balances microbial demands during decomposition. Soil Biol. Biochem., 53, 133-141.

Moorhead D., Lashermes G., Recous S., Bertrand I., 2014. Interacting microbe and litter quality controls on litter decomposition: a modeling analysis. PLoS One, 9, e108769.

Parisey N., Bourhis Y., Roques L., Soubeyrand S., Ricci B., Poggi S., 2016. Rearranging agricultural landscapes towards habitat quality optimisation: in silico application to pest regulation. Ecological Complexity, 28, 113-122.

Pelzer E., Fortino G., Bockstaller C., Angevin F., Lamine C., Moonen C., Vasileiadis V., Guerin D., Guichard L., Reau R., Messean A., 2012. Assessing innovative cropping systems with DEXiPM, a qualitative multi-criteria assessment tool derived from DEXi. Ecol. Indicators, 18, 171-182.

Picault S., Huang Y.-L., Sicard V., Beaudeau F., Ezanno P., 2017. A multi-level multi-agent simulation framework in animal epidemiology. In: 15th International Conference on Practical Applications of Agents and Multi-Agent Systems (PAAMS'2017), juin 2017, Porto, Portugal. Advances in Practical Applications of Cyber-Physical Multi-Agent Systems: The PAAMS Collection (Demazeau Y., Davidsson P., Bajo J., Vale Z., eds), Springer, 209-221.

Roques L., Bonnefon O., 2016. Modelling population dynamics in realistic landscapes with linear elements: a mechanistic-statistical reaction-diffusion approach. PLoS One, 11, e0151217.

Sadok W., Angevin F., Bergez J.-E., Bockstaller C., Colomb B., Guichard L., Reau R., Messean A., Doré T., 2009. MASC, a qualitative multi-attribute decision model for ex ante assessment of the sustainability of cropping systems. Agron. Sustain. Dev., 29, 447-461.

Soubeyrand S., 2016. Contributions to Statistical Plant and Animal Epidemiology, Mémoire d'HDR, Université d’Aix-Marseille. 



\section{Contribution des}

\section{agroéquipements et du}

\section{numérique à l’agroécologie :}

\section{renforcer la prise}

\section{en considération du vivant}

Divers DOCUMENTS D'ORIENTATION DE LA RECHERCHE abordent la place des agroéquipements sensu lato dans le contexte de l'adaptation de l'agriculture aux nouveaux enjeux. Les rapports «Agriculture et innovation 2025 » (Bournigal et al., 2015) ou « Agroéquipements et triple performance : freins et leviers pour la transition agroécologique » (Machenaud et al., 2014) proposent des actions ciblées sur le numérique et la robotique agricole pour porter l'ambition d'une performance élargie sur les dimensions économiques, environnementales et sociales. La logique est simple : si par une meilleure connaissance des besoins des plantes et des animaux, on peut moduler au plus juste les interventions et les apports d'intrants, alors le système sera plus vertueux car plus économe, évitant les gaspillages et les pollutions inutiles, plus favorable à l'environnement car moins dispendieux en énergie fossile et générateur de moins de fuites, plus juste socialement car réduisant la pénibilité du travail, mieux adapté aux spécificités de chaque situation, plus en phase avec les cahiers des charges, les circuits courts, les attentes des consommateurs. Ces documents se font l'éloge de la technologie, porteuse de progrès.

Paradoxalement, la capacité du numérique et des agroéquipements à porter spécifiquement un modèle d'agriculture agroécologique reste un pari, car ces évolutions technologiques peuvent aussi renforcer une industrialisation plus poussée de l'agriculture, dans la continuité de soixante ans de développement dans la visée d'un gain de productivité. Il est donc nécessaire de réfléchir spécifiquement aux apports possibles des agroéquipements et du numérique aux démarches agroécologiques. Pour que l'agroécologie tire bénéfice de ces technologies, il est primordial qu'elle outille la biologie et valorise les hétérogénéités des milieux rencontrées à différentes échelles. Alors que l'agriculture cherchait à masquer les hétérogénéités pour aller vers une standardisation des pratiques culturales, l'agroécologie vise en effet à tirer profit des conditions locales pour mieux les valoriser, pour répartir les risques et les complémentarités entre animaux ou entre cultures. La technologie peut, si elle est utilisée convenablement, être une source majeure de progrès (Bellon-Maurel et Huyghe, 2017), voire parée de multiples vertus. Toutes les agricultures du monde n'y ont pas accès, tous les agriculteurs ne sont pas équipés, toutes 
les avancées possibles ne sont pas réalisées. À titre d’illustration, la technologie des pulvérisateurs offre des équipements dont la performance appuyée par le numérique permet de réaliser des économies de produit phytosanitaire sans réduction de l'efficacité (encadré 6.1). Pour autant, si cela relève pleinement de l'agriculture de précision ${ }^{57}$, les processus agroécologiques ne sont pas mobilisés.

\section{Encadré 6.1. La maîtrise technologique de la pulvérisation.}

La maîtrise technologique de la pulvérisation permet de moduler la dose apportée à la vitesse d'avancement, de définir des tronçons en dessous de la zone à traiter, pour lesquels elle ajuste automatiquement la hauteur de la rampe à celle de la végétation pour éviter les zones de double traitement ou les trous. Le mélange de l'eau et du produit de traitement est réalisable en temps voulu ; le bouchon standardisé vient se visser et se dévisser sur le pulvérisateur sans nécessiter aucun contact ; des mouchards enregistrent l'intervention et pré-remplissent les fichiers informatisés de suivis. Des récupérateurs ou dispositifs permettent de mieux confiner la pulvérisation pour qu'elle ne soit pas dispersée; des buses antidérive limitent les pertes hors des zones ciblées. Par un couplage entre l'état de la végétation et la détection des ravageurs, l'intervention est mieux ciblée sur la présence avérée du risque phytosanitaire et des conditions météorologiques pour que le traitement soit optimal, et donc les doses appliquées les plus faibles possible. Sans même cumuler toutes ces avancées et à condition d'y mettre le prix, des réductions d'intrants phytosanitaires sont possibles dans une fourchette de 10 à $30 \%$ sans baisse d'efficacité. Qui pourrait dénoncer les bienfaits de cette tendance ? D'autant que la majorité du parc des pulvérisateurs reste obsolète et présente pour plus de deux tiers d'entre eux le réglage de sortie usine, c'est-à-dire sans adaptation aux conditions locales. II paraît de plus difficile de dire que l'information et les savoirs nécessaires n'ont pas été délivrés, puisqu'en France les agriculteurs ont tous suivi et obtenu leur Certiphyto au cours des cinq dernières années.

Donc discuter d'agroéquipement pour l'agroécologie n'a vraiment de sens que si celle-ci en ressort enrichie ou facilitée. Nous suggérons que le rôle majeur de la technologie doit être de faciliter le croisement explicite de plusieurs disciplines. En effet, l'agronomie, l'écologie, les sciences de gestion et la géographie ne sont encore que très marginalement embarquées dans cette avancée technologique, portée avant tout par les sciences de l'ingénieur, l'algorithmique, la robotique, la géolocalisation par guidage, la connexion sans fil de capteurs physiques, le couplage des informations dans un système pilotant des électrovannes.

57. L'agriculture de précision vise principalement l’optimisation d'intrants essentiellement externes, quand l'agroécologie envisage plutôt leur internalisation au système, leur intégration dans l'agroécosystème rendu plus autonome, leur disponibilité, ce qui nécessite de gérer les flux et de reconstituer les stocks, et leur efficience, ce qui suppose leur fonctionnalité pour assurer leur potentiel de mobilisation. 
Les agroéquipements, et plus largement ce qui entre dans le cadre des outils pour l'action (outils d'aide à la décision, tableaux de bord, prévisions, cartographie...), aident au pilotage et à son appropriation par le plus grand nombre. On comprendra donc que le rapprochement des termes « agroécologie » et « agroéquipements » cerne, en fait, la fourniture des moyens, outils de pilotage et d'accompagnement, pour aider à l'adoption de pratiques à visée agroécologique, à travers un suivi fin du fonctionnement dynamique de l'agroécosystème. Ce thème renvoie donc assez largement à l'acquisition d'informations et à la chaîne de traitement associée, à même de piloter des flux, dans sa facette finalisée, pour que l'agriculture puisse gagner en durabilité à travers le déploiement d'une ingénierie agroécologique.

\section{Des avancées scientifiques récentes}

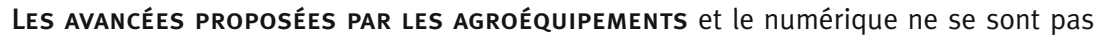
cantonnées à l'agriculture des pays tempérés, ni à la seule agriculture conventionnelle. Elles concernent toutes les agricultures. Une technologie en support à l'agroécologie peut être définie par sa contribution à informer ou à contrôler les processus qui soustendent les principes de l'agroécologie :

- rendre visibles et intégrer la réalité et les mécanismes déclencheurs de stimulation des défenses internes, comme en partie prôné par la biodynamie ;

- quantifier les régulations naturelles, en partie mises en exergue par l'agriculture biologique ;

- évaluer l'occupation des niches écologiques et leur préservation, en partie préconisées par l'agriculture de conservation et l'agroforesterie ;

- tirer profit de la valorisation des complémentarités entre organismes pour réduire la compétition et créer des synergies, prônée par l'agriculture biologique, la biodynamie, l'agriculture de conservation...;

- reconstituer les stocks et maintenir les paramètres de l'environnement dans une gamme de fonctionnement qui respecte les particularités locales de l'environnement et ses potentialités, mobilisant pour se faire le bouclage des cycles à travers la valorisation de la biomasse, mais aussi le recyclage, la sobriété et l'efficience des ressources mobilisées.

Nombre de ces connaissances ne peuvent être prises en compte que si des agroéquipements dédiés les exploitent pour moduler leur action ; valoriser les complémentarités entre plusieurs espèces végétales au sein d'une même parcelle nécessite par exemple que les moissonneuses puissent collecter une récolte dans un peuplement composite.

Les enjeux cognitifs des agroéquipements pour l'agroécologie touchent ainsi la caractérisation du fonctionnement de l'agroécosystème, la couverture des régulations biologiques, l'estimation du potentiel de mobilisation de processus tels que la prédation, la libération d'éléments fertilisants par dégradation de la matière organique, la capacité de stockage de carbone, le potentiel de rétention d'eau (Dobriyal et al., 2012), la 
mobilisation de mécanismes d'immunité et de résilience des organismes. Ces processus impliquent la maîtrise de la chaîne qui passe de la collecte d'informations à sa traduction dans une capacité de modulation, par des outils dédiés aux processus biologiques sousjacents. Plus largement, ils impliquent des articulations avec les secteurs de l'amont et de l'aval de l'agriculture, dans le choix des espèces et des variétés, le pilotage modulé des hétérogénéités et la traçabilité, des modes de production jusqu'au consommateur.

\section{Le croisement du machinisme numérique et de l'agroécologie, un espace à explorer}

L'intersection d'un secteur recouvrant l'agroécologie puis son extension à l'ingénierie écologique en agriculture d'un côté, et le machinisme étendu à la tractorisation, la robotique, l'imagerie, les capteurs et l'ensemble de l'ingénierie mécanique en agriculture de l'autre, recouvrent quelques centaines de publications, soit moins de 0,01\% des références des sciences mécaniques. Le contenu de cette intersection fait ressortir quelques thématiques autour de la prévision météorologique, la place de la télédétection et de la proxi-détection dans la caractérisation des états physiques du milieu et de la diversité des situations, dans la caractérisation des hétérogénéités à différentes échelles, dans le suivi et les effets du tassement sur l'activité biologique et le fonctionnement des sols, le pilotage de l'irrigation.

Du point de vue méthodologique, une large place est occupée par l'usage des techniques d'apprentissage automatique appliquées à de gros jeux de données acquis sur des enjeux préexistants et dont certains relèvent de préoccupations agroécologiques. Ainsi, l'ajout d'une composante disciplinaire en machinisme ou en sciences de l'ingénieur au croisement des disciplines fondatrices de l'agronomie et de l'écologie amène à viser des communautés scientifiques qui n'ont a priori que peu d'intérêts communs. En première instance, il semble que nombre de travaux scientifiques en agroécologie se suffisent d'entrées monodisciplinaires. Vue à travers les outils et les approches de pilotage de l'ingénierie, l'agroécologie est encore dans une phase d'appropriation des briques disciplinaires plus que dans le déroulement d'objets majeurs et interdisciplinaires qui lui soient originaux.

Ce relatif no man's land scientifique contraste avec les nombreuses conventions qui toutes poussent à déployer rapidement l'agroécologie. Sivakumar et al. (2000) en ont mené l'analyse et citent « the United Nations Framework Convention on Climate Change (UNFCCC), the Convention on Biological Diversity (CBD) and the United Nations Convention to Combat Desertification (UNCCD)... The World Food Summit Plan of Action (WFSPA), which was developed in 1996, includes several commitments to make agricultural production sustainable ${ }^{58}$. Le flot des conventions ne s'est pas tari depuis, et la FAO a notamment investi le thème ${ }^{59}$, sans que le rôle que peuvent jouer les agroéquipements et les sciences du numérique dans la mise en place de mesures en faveur de la durabilité soit abordé.

58. Voir https://unfccc.int/ ; https://www.cbd.int/ ; https ://www.unccd.int/ ; http://www.fao.org/3/ w9990e/w9990e07.htm.

59. Voir www.fao.org/agroecology/fr/. 


\section{Diagnostiquer le potentiel de mobilisation des régulations biologiques}

Les régulations biologiques constituent un pilier de l'agroécologie. Pour autant, il reste difficile de qualifier l'état de ces régulations ou de leur potentiel de mobilisation. Actuellement, pour décider du bien-fondé d'intervenir, les agriculteurs peuvent avoir recours à des pièges (connectés ou non) qui vont informer précocement de la démographie d'un ravageur majeur (par exemple, cuvette jaune pour quantifier les méligèthes ou usage de phéromone pour capturer certains lépidoptères). Cependant, une importante littérature scientifique construite sur la relation prédateur-proie, sa prédictibilité et son fonctionnement cyclique peine encore à se traduire dans des pratiques en faveur de la fixation de populations d'auxiliaires prédateurs. Certaines infrastructures agroécologiques telles que les haies peuvent jouer un rôle de maintien des auxiliaires en apportant « le gîte et le couvert ». On peut imaginer qu'une partie des actions de biocontrôle consiste à quantifier en temps réel le potentiel de prédation et couple l'observation à un lâcher éventuel de ressources à même de maintenir des prédateurs à des densités élevées. Il manque actuellement toute la chaîne d'équipements adaptée pour évaluer les besoins des auxiliaires, réaliser le conditionnement et le lâcher des ressources alimentaires susceptible de stabiliser sur la parcelle la population d'auxiliaires en couvrant une période de disette qui conduirait sinon à ce qu'elle quitte massivement la parcelle.

\section{Gérer les hétérogénéités, moduler les risques}

L'agroécologie, loin d'orienter les espèces et les systèmes vers une spécialisation et une sélection univoque, promeut la variabilité et la diversité comme moyen d'accroître la performance des systèmes face à un environnement moins artificialisé par différents intrants susceptibles de déséquilibrer les écosystèmes.

Il coexiste actuellement deux visions de la prise en compte des hétérogénéités. Dans la première, la connaissance des hétérogénéités appelle une action différenciée pour mieux les absorber, les niveler et offrir une production la plus homogène possible en phase avec les standards du marché. Dans la seconde vision, les hétérogénéités signent l'existence sous-jacente d'une variation donnant lieu à une réponse différenciée. Tenir compte des hétérogénéités revient alors à adopter une démarche qui entretienne la meilleure adaptation possible à chacune des dimensions du gradient observé. Dans cette seconde vision, la prise en considération des interactions génétique $\times$ environnement $\times$ conduite vise plutôt à entretenir, voire à amplifier la différenciation. Elle est vue comme un gage de meilleure répartition des risques, de moindre concurrence entre individus, de complémentarités et d'offres d'une réponse biologique adaptée aux conditions du milieu. Il est à peu près certain qu'à brève échéance, le déploiement des pratiques agroécologiques signera la fin du paradigme d'un troupeau homogène mené avec une règle unique pour l'ensemble de la cohorte et d'une seule culture d'une variété unique par parcelle et par an. Actuellement, les agroéquipements commencent tout juste à savoir gérer cette diversité locale. 


\section{Quelques exemples}

\section{Visualiser le " stress " des plantes et des animaux}

L'agroécologie met en exergue le réseau d'interactions entre organismes qui gravitent autour de la plante cultivée ou de l'animal d'élevage et modifient positivement ou négativement l'état sanitaire de l'élevage ou de la culture à travers des grandeurs mesurées mobilisables pour son pilotage. Alors que les physiologistes maîtrisent de mieux en mieux la connaissance des voix métaboliques affectées par tel ou tel stress, on ne dispose pas encore d'un moyen fiable, utilisable en routine et si possible non destructif, pour caractériser au moment opportun l'état de stress des animaux ou des plantes. De telles informations seraient pourtant directement exploitées par des agroéquipements venant apporter des actions correctives de manière localisée dans le temps ou l'espace. Cet ajustement dépasserait le stade actuel de l'agriculture de précision, en apportant cette précision sur la base d'une information d'origine biologique. Déjà, les progrès d'analyse des signaux en lien avec le déficit azoté des plantes permettent d'envisager un pilotage de la fertilisation qui soit basé sur l'expression des besoins par la plante elle-même, en lieu et place d'un schéma de fertilisation prédéfini en début de saison. La caractérisation de la couverture en continu des besoins azotés des cultures autorise déjà différentes pistes d'optimisation, dont une traduction largement diffusée est la cartographie des hétérogénéités au sein des parcelles par drone. Une mesure des carences évaluée directement sur les plantes compléterait utilement l'analyse des besoins pour conduire des pilotages au plus près de ce que les plantes peuvent absorber et valoriser (Ravier, 2017). Le pilotage dynamique de la fertilisation, en remplacement d'une approche prédictive, peut être considéré comme emblématique de la dimension agroécologique car il repose sur une information, en dynamique, des besoins physiologiques, et donc sur l'expression du vivant.

En outre, la maîtrise de l'état physiologique des cultures pourrait étayer la réalité et les mécanismes sous-jacents de certaines pratiques jugées encore peu conventionnelles, comme celles pratiquées en biodynamie qui revendiquent de stimuler les défenses naturelles des plantes, mais sans que l'on ait pu établir de manière formelle la réalité de leur mise en place et les conditions propices à leur réussite.

Si les avancées sont notoires, y compris dans la mise au point de normes, la transformation de ces avancées de laboratoire dans des dispositifs plus ou moins automatisés ou rendus accessibles au plus grand nombre reste à mener. Comment rendre compte de l'état réversible de carence d'une plante pour un pilotage dynamique de la fertilisation ? Cette question constitue un cas d'école de ce qu'il faudrait rendre accessible aux agriculteurs.

\section{Vers un usage des outils de phénotypage à large échelle}

La dynamique est portée, du côté végétal, par le projet Phenome soutenu par le programme d'investissements d'avenir, et, du côté animal, par différents projets (projet 
Casdar Morpho3D de Phénotypage morphologique par imagerie 3D, projets européens SmartCow ou Aquaexcel2020). Ces travaux mobilisent des unités de recherche comme les installations et les unités expérimentales en vue d'avancer vers trois innovations majeures : - la quantification et la qualification des caractéristiques de robustesse, de résilience et de plasticité que l'agroécologie peut mobiliser ; c'est un enjeu particulièrement important en élevage, afin de mieux caractériser la propriété de résilience, peu étudiée jusqu'à présent faute de phénotypage à haut débit, et d'intégrer explicitement, sur tout ou partie de la durée de vie de l'animal, la santé et le système immunitaire, l'état des réserves et leurs variations avec l'imagerie $3 \mathrm{D}$, la capacité à se reproduire ;

- la prise en compte des hétérogénéités au sein des parcelles et des troupeaux, que l'on peut non seulement caractériser, mais piloter de manière différenciée ; une retombée possible à cette caractérisation vers une meilleure résilience face aux aléas, une meilleure articulation entre la performance et la durabilité des exploitations agricoles ; - la prédiction génomique de la valeur des individus qui va se développer rapidement, en élevage bovins par exemple. Elle ouvre la possibilité de prédire et donc d'intégrer de nouveaux caractères. Il s'agit d'amplifier la caractérisation des traits recherchés et leur mesure plus ou moins automatisée sur des pas de temps permettant l'intégration et l'articulation aux informations génomiques collectées ou modélisées par ailleurs. En gérant de manière différente les individus au sein d'un troupeau, on peut mieux étaler les risques, mais aussi diminuer la compétition entre individus en jouant sur leur complémentarité. Il peut ne pas y avoir d'incidence négative pour la collecte et la mise en marché dès lors que la traçabilité est renforcée.

\section{\ Avancées en ingénierie agroécologique pour les cultures sous abri}

Un set de techniques d'ingénierie agroécologique se déploie en culture sous abri, avec la gestion des ravageurs par solarisation, aspiration, pièges, lâchers d'auxiliaires, plantes pièges, usage de plantes relais, fermetures des ventaux en cas de signalisation de nuages de spores avec risque de contaminations aériennes, capture d'une partie des rayonnements solaires, etc. Ces pratiques d'ingénierie nécessitent souvent des équipements dédiés et couvrent une gradation de pratiques diversement mobilisatrices de la biodiversité, favorables à l'environnement et à la santé. L'unité mixte technologique FioriMed ${ }^{60}$ et une plateforme d'accompagnement ont été créées, complétées par quelques ouvertures via des applications pour Smartphone (voir notamment la série des applications pour mieux identifier, connaître, maîtriser Di@gnoPlant mise au point à Bordeaux $\left.{ }^{61}\right)$. On notera aussi l'articulation à l'enseignement avec différents modules de l'UVED ${ }^{62}$, notamment EcoHort, «La conception de systèmes horticoles écologiquement innovants ». Dans

6o. Voir https://www.umt-fiorimed.fr/

61. Voir http://ephytia.inra.fr/fr/C/23654/Veg-Di-g-Applications-web-et-smartphones.

62. Université virtuelle Environnement et Développement durable. Voir l'Université virtuelle d'agroécologie (UVAE) sur https://www6.inra.fr/uvae/Ressources-UVAE/Types-de-modules/Ingenierie-agroecologique. 
tous les cas, l'expertise et le savoir-faire dispensés sont très orientés par et sur le fonctionnement biologique sensu lato. Cette posture autorise des approches a priori plus vertueuses et durables, faisant elles-mêmes appel à des agroéquipements quand cela s'avère nécessaire. Mais le lien aux équipements et aux outils d'accompagnement reste plus indirect que direct.

\section{Les questions de recherche}

\section{Des outils et des approches pour valoriser la biodiversité dans son contexte local}

Les approches antérieures de modernisation de l'agriculture en milieu tempéré ont largement contribué à simplifier la conduite par une plus grande homogénéisation des entités de production tant animales que végétales, facilitant la diffusion généralisée des voies de progrès. La conduite de systèmes agroécologiques suscite un intérêt croissant pour valoriser la diversité intra ou interspécifique comme un moyen privilégié d'accroître les capacités de résilience et d'efficience des systèmes de production. Ces derniers peuvent fonctionner sur une base très productive, à condition qu'une partie de la biomasse reste sur place pour soutenir l'activité biologique et permettre le maintien des chaînes trophiques. Cette conduite agroécologique cherche aussi à mobiliser les caractéristiques et les propriétés propres de l'environnement local, comme autant de conditions sur lesquelles s'appuyer pour accroître la durabilité du système mis en place. Ceci plaide pour un phénotypage en continu et assez large des cultures, des animaux d'élevage et des composantes majeures de la biodiversité naturelle, hébergée pour englober la couverture des besoins ainsi que les fonctionnalités auxquelles tous contribuent. Déjà, de nombreux travaux visent à mieux identifier les propriétés et à tirer parti de cette double source de diversité, externe (l'environnement local) et interne (ce que l'on y introduit et que l'on pilote) (encadré 6.2). Cela nécessite une articulation fine avec les modèles de simulation des effets des interactions biotiques sur la valeur phénotypique afin d'aider à un pilotage approprié des systèmes, thème traité dans le chapitre 5 .

Des travaux pilotes de valorisation de la biodiversité dans des couverts hétérogènes existent : agroforesterie, associations végétales, y compris en prairie et en forêt mixte, mélanges variétaux, etc. La caractérisation des propriétés résultantes de robustesse face aux aléas, de productivité, de facilité de gestion aussi (comme une simple destruction d'un couvert par roulage), etc., est souvent au cœur des travaux conduits ou à mener. Des capteurs, des campagnes de mesures, de la cartographie y sont davantage mobilisés que des (agro)équipements au sens strict, mais on peut anticiper que les agroéquipements futurs feront un « usage embarqué » des éléments de diagnostic se traduisant par une modulation des réglages et une différenciation des actions (cas d'un désherbage sélectif de la culture). 
Encadré 6.2. Gérer la diversité végétale à des échelles fines : importance des technologies densimétriques ou optiques de tri.

L'équipement des exploitations ou des Coopératives d'utilisation de matériel agricole (CUMA) avec des trieurs mobilisant différentes technologies densimétriques ou optiques ouvre des champs d'applications mettant à profit des processus agroécologiques. Deux moments phares sont visés : une action prophylactique en amont du semis pour des semences de ferme, et une action d'allotage post-récolte pour atteindre les standards d'homogénéité exigés par les marchés et les technologies de transformation. Avec ces équipements, il devient possible de pratiquer les associations d'espèces et de variétés adaptées aux conditions locales dans une majorité de parcelles. Ce type de culture valorise la biodiversité, limite les risques épidémiques et s'adapte mieux aux particularités météorologiques de la saison culturale. Si ce qui sera récolté n'est pas bien maîtrisé au moment du semis d'un mélange plurispécifique, en revanche, la probabilité d'une récolte est fortement accrue. Plusieurs filières de transformation maîtrisent l'ajustement du processus industriel à la nature de la matière première travaillée : de l'alimentation animale à la production de bière, etc. En fin de compte, outiller la capacité de tri, c'est permettre de gérer la diversité que prône l'agroécologie tout en restant en phase avec les attentes et les besoins des marchés.

\section{Quantifier les services écosystémiques des agroécosystèmes et les valoriser}

La quantification des services écosystémiques ouvre la possibilité de « paiements pour services rendus ». Plus généralement, mieux caractériser l'environnement, identifier des grandeurs mesurables simples qui rendent compte de son fonctionnement participent de la durabilité même des agroécosystèmes. Cette quantification sera d'autant plus nécessaire que l'on passera, pour le versement d'aides conditionnelles, d'une logique d'obligation de moyens à une logique d'obligation de résultats. Derrière la stratégie de la reconnaissance des services écosystémiques rendus par les agrosystèmes, ce sont les questions de la certification des actions mises en œuvre, de l'évaluation multicritère et de l'internalisation des externalités, en lien avec les choix de conduites agronomiques, qui sont ciblées. Développer l'agroécologie concerne donc la mesure de l'efficacité des outils (incitatifs ou réglementaires) des politiques publiques et, in fine, la manière de caractériser la bonne couverture des exigences des cahiers des charges, ouvrant droit à une reconnaissance financière ou autre.

Ce domaine fait largement appel aux préoccupations des projets de recherche sur les services écosystémiques, mais sous l'angle de l'appui à la mesure et au pilotage, éventuellement à la couverture assurantielle des risques. Là aussi, si on se limite à l'entrée par la mesure et le pilotage, les travaux éligibles et reconnus sont peu nombreux. C'est donc une priorité scientifique que d'amplifier ces recherches, de les rendre plus visibles et plus aisément mobilisables. 


\section{Mobiliser la boucle " capter-actionner " pour attacher le diagnostic à une action dynamique}

Différents champs scientifiques sont mobilisés pour aller du diagnostic à l'action. On en cite classiquement quatre qui concernent la prise de mesure, son interprétation, la prise de décision et la réalisation de l'action. Chacune des étapes peut mobiliser des expertises, des approches et des outils variés. Les communautés scientifiques qui explorent ces différentes étapes ont leurs propres standards, et la capacité de dialoguer entre les outils mobilisés devient alors un point d'attention majeur. Garantir l'articulation entre l'observation interprétée et l'action dépend des métriques employées, les sorties d'une étape devant être les entrées de la suivante. En agroécologie, du fait même de vouloir s'affranchir au mieux de l'artificialisation du milieu et de l'apport d'intrants exogènes, c'est le déroulement même du processus écologique qui doit être mis en avant plutôt qu'un état absolu : est-on en train de stocker ou de déstocker du carbone ? Le potentiel des régulations est-il mobilisable rapidement ? Etc. Les processus agroécologiques sont par essence dynamiques : ce sont des flux de matière et d'énergie et des changements d'état. Il y a donc lieu de choisir des grandeurs mesurables adaptées non pas pour rendre compte du franchissement de seuils, mais pour donner la valeur (positive ou négative) d'une tendance, autrement dit des grandeurs bâties comme des trajectoires, ou tout au moins des écarts entre situations. L'agroécologie étant construite sur des systèmes complexes s'appuyant largement sur la fonctionnalité des interactions entre organismes, les métriques de l'agroécologie font la part belle à des mesures relatives, des "deltas ». Force est de constater qu'il reste beaucoup à faire d'un point de vue scientifique pour alimenter cette approche dynamique de l'agriculture. Il s'agit donc d'instruire de quelle manière les nouvelles technologies sont en mesure d'offrir des perspectives prometteuses pour contribuer, depuis la télédétection à haute résolution jusqu'à des capteurs in situ, à caractériser l'environnement et ses hétérogénéités, à suivre les trajectoires de grands processus biologiques impactant la mise en place des composantes du rendement, le bouclage des cycles, la régulation de la ressource en eau en quantité et qualité, la dynamique d'entrée et de sortie des flux.

Pour tous ces attendus, c'est plus « le delta » entre deux points ou entre deux dates qui devient l'objet d'attention que la valeur absolue, sauf si cette dernière conduit, via des effets seuils, à des points de basculement du fonctionnement du système. Ces questions renvoient notamment aux besoins d'évaluation de l'activité biologique (Wells et al., 2013) et plus largement des services écosystémiques, ceux-ci pouvant donner lieu à des aides financières conditionnées au service rendu. Il est encore relativement difficile de savoir quels besoins d'équipement pour l'analyse et la compréhension des processus biologiques sous-tendant la réalisation des services écosystémiques sont nécessaires.

La masse d'informations à gérer complique le travail du décideur si des outils performants ne sont pas développés pour intégrer ces informations dans le pilotage. On peut penser que les attentes couvrant le potentiel des interactions génétique $\times$ environnement $\times$ conduite constituent à elles seules un véritable secteur de recherche innovant 
et encore peu abordé. L'incapacité à en rendre compte serait susceptible de décrédibiliser la production d'une information riche, mais avec laquelle le décideur ne sait pas que décider. Par exemple, comment intégrer l'information de prévision de la performance génomique des animaux dans une conduite individualisée adaptée, et ce en fonction des différents environnements, pour tirer parti de cette diversité ? Comment combiner ces flux d'informations dynamiques sur les différents phénotypes et génotypes pour décider des opérations les plus pertinentes à des échelles fines (individus ou petites surfaces) ? La question de la valeur ajoutée ou apportée par l'interprétation de ces données, traduite en règles de décision, est bien au cœur des questions de recherche. On peut approfondir aussi bien la mesure que le pilotage, mais la recherche sera attendue sur l'éclairage et la contribution à la distinction entre « outil pour phénotyper » et « outil pour piloter».

La question de l'accès aux données, des droits et des devoirs concernant leur utilisation, leur réutilisation et leur diffusion peut conduire à faire appel à des compétences juridiques pour anticiper les difficultés susceptibles de fragiliser les développements technologiques.

\section{Les partenariats socioprofessionnels selon les enjeux d'application}

Les méthodologies à maîtriser peuvent être regroupées en cinq grands secteurs qui structurent aussi cinq champs de partenariats socioprofessionnels encore largement indépendants.

1. Ce qui relève de la caractérisation de l'environnement, des plantes ou des animaux d'élevage, dans l'optique de mieux piloter, mieux analyser, mieux reprendre la main sur les processus, les actes associés. La finalité d'améliorer la fertilité des sols et d'atténuer le changement climatique relève de cette logique.

2. Ce qui relève du partage d'information entre acteurs des territoires pour déployer les logiques agroécologiques qui s'expriment à l'échelle supra-exploitation sur la caractérisation du risque épidémiologique, le rapprochement offre-demande, l'articulation aux éléments amont-aval.

3. Les agroéquipements pour les besoins spécifiques de l'agroécologie et des conduites que cela mobilise (de type trieur de semences, récolte sélective, et autres équipements de gestion des haies ou des arbres isolés).

4. La caractérisation de la réponse des organismes à des fins de phénotypage et de sélection. Le numérique va profondément remanier les réseaux d'expérimentation et d'observation.

5. Les éléments de la traçabilité des modes de conduite pour une différenciation par la collecte, la transformation et le marché. L'ambition de développer et de diffuser les outils d'évaluation multicritère des systèmes agricoles et alimentaires jusqu'au consommateur en dépend largement. 
Le rapport «Agriculture et innovation 2025 » (Bournigal et al., 2015) se positionnait déjà sur ces différents champs. Beaucoup de ces travaux pourraient s'engager dans les unités expérimentales qui ont encore un faible niveau de numérisation et un déficit de situations de pilotage de processus en dynamique, en dehors des digesteurs et des chambres de culture. On perçoit notamment la nécessité d'intégrer l'ensemble des mesures de la performance pour en faire un modèle économique attractif, et cela ne va pas spontanément de soi. Couvrir cet attendu est sans doute la contribution que la recherche peut et doit apporter au sein de ses possibles partenariats.

Par ailleurs, la distinction entre mesurer pour connaître et mesurer pour piloter concentre quelques défis majeurs pour rendre les avancées effectives. Si on peut facilement identifier et rendre compte de l'activité biologique d'un sol dans une campagne de mesure pour une expérimentation dédiée, il n'en va pas de même pour imaginer comment cette connaissance peut être mobilisée dans une action de modulation des pratiques, dans l'évaluation du risque de certaines impasses, dans la manipulation de facteurs correctifs pour accroître ou diminuer la dynamique observée : enfouissement d'un engrais vert, mobilisation du priming effect pour lancer la minéralisation de la matière organique en phase avec la capacité d'absorption par les plantes des nutriments libérés, action via des facteurs physiques et éventuellement chimiques, modification des communautés microbiennes, de microfaune ou de macrofaune par ajout d'organismes à fort pouvoir modificateur direct (compétition) ou indirect (aération, pouvoir drainant augmenté, acidification réduite, etc.). Un Carrefour de l'innovation agronomique a fait le point récemment sur ces questions $^{63}$. Ce qu'il faut déployer pour piloter le microbiote d'un sol s'applique sans doute de manière similaire à la flore intestinale des ruminants. Ces besoins d'intégration entre l'adéquation des leviers mobilisés et la réponse du vivant peuvent nécessiter d'y consacrer une partie des efforts dans les arènes où la coconception est de mise. La possibilité d'orienter des systèmes agroécologiques généralement complexes tout en gardant la simplicité de leur pilotage sera à ce prix.

\section{Références bibliographiques}

Bellon-Maurel V., Huyghe C., 2017. Putting agricultural equipment and digital technologies at the cutting edge of agroecology. OCL, 24, D307.

Bournigal J.-M., 2014. Définir ensemble le futur du secteur des agroéquipements. Rapport de la mission agroéquipements, Irstea.

Bournigal J.-M, Houllier F., Lecouvey P., Pringuet P., 2015. Agriculture et Innovation 2025 : 30 projets pour une agriculture compétitive et respectueuse de l'environnement. Rapport aux ministres en charge de l'agriculture et en charge de la recherche.

Dobriyal P., Qureshi A., Badola R., Hussain S.A., 2012. A review of the methods available for estimating soil moisture and its implications for water resource management. J. Hydrol., 458, 110-117.

63. https://www6.inra.fr/ciag/Revue/Volumes-publies-en-2018/Volume-69-Novembre-2018. 
Machenaud G., Klein P., Terrien F., Pasco E., 2014. Agroéquipement et triple performance. Freins et leviers pour la transition agroécologique, ABSO conseil.

Ravier C., 2017. Conception innovante d'une méthode de fertilisation azotée : Articulation entre diagnostic des usages, ateliers participatifs et modélisation. Thèse de doctorat, AgroParisTech, 203 p.

Sivakumar M.V.K., Gommes R., Baier W., 200o. Agrometeorology and sustainable agriculture. Agric. Forest Meteorol., 103, 11-26.

Wells D.M., Laplaze L., Bennett M.J., Vernoux T., 2013. Biosensors for phytohormone quantification: challenges, solutions, and opportunities. Trends Plant Sci., 18, 244-249. 



\section{Conclusions}

LES SIX CHAPITRES DE CET OUVRAgE METTENT EN ÉVIDENCE des convergences autour des enjeux et des questions de recherche en agroécologie : l'étape d'appropriation des principes de l'agroécologie et des conséquences sur la manière de concevoir les recherches n'est pas encore achevée ; la dimension d'assemblage des connaissances qui caractérise l'agroécologie apparaît désormais de manière évidente comme très interdisciplinaire ; des enjeux transversaux sont identifiés autour des données, des modèles, des outils pour la (re)conception et le pilotage des activités agricoles, la prise en considération des effets directs et différés d'une transition agroécologique. Un décloisonnement et une mise en cohérence des compétences sont quoi qu'il en soit des conditions nécessaires pour avancer sur les six thèmes développés, et il s'agit pour cela de mobiliser les compétences existantes, en les associant autour de problématiques d'agroécologie, de trouver et/ou de former des " assembleurs » de compétences et d'identifier les domaines transversaux qui nécessitent un renforcement. Il s’agit par exemple de développer les compétences sur les interactions entre génétique et environnement, entre environnement et alimentation, de la conceptualisation et l'analyse des socio-écosystèmes, de la modélisation bioéconomique, etc. Dans tous ces domaines, l'ambition est de promouvoir une vision systémique. Recruter et former dans le champ de l'agroécologie est important, mais il faut sans doute encore plus favoriser l'implication de chercheurs thématiciens dans des démarches interdisciplinaires aux interfaces entre agriculture, environnement et alimentation, aux interfaces entre sciences biotechniques et sciences sociales.

\section{Diversité et diversification : observer, traduire, piloter}

SYSTÈMES DE PRODUCTION AGROÉCOLOGIQUE, diversité et diversification sont associés à différentes échelles (génotypes, espèces élevées/cultivées, échelle intraparcellaire et interparcellaire, allongement et diversification des rotations, mode de conduite des cultures, systèmes de transformation, systèmes alimentaires...). L'une des conséquences de cette diversité et de cette diversification est l'augmentation de l'hétérogénéité à tous les niveaux, jusqu'à des aspects qualitatifs des produits agricoles eux-mêmes. Plusieurs fronts de recherche sont identifiés sur cette base : considérer dans la sélection et la production plus de niveaux d'interaction, et, découlant de là, plus de diversité dans les produits agricoles et alimentaires. L'enjeu est d'en tirer parti, depuis la diversité initiale de la matière première jusqu'à l'élaboration des produits à finalité alimentaire comme non alimentaire, satisfaisant l'utilisateur final. C'est l'occasion de réfléchir à la traçabilité des caractéristiques des productions et leur couplage à la pratique d'association de lots complémentaires au moment de la transformation. Cette hétérogénéité des produits nécessite de retravailler ce que 
recouvrent les normes et les standards. Ceux-ci ont été élaborés pour garantir un niveau de qualité à l'utilisateur final, ce qui s'est souvent traduit par une homogénéisation des produits. Comment les normes peuvent-elles désormais accompagner de manière nouvelle la transition agroécologique ? Les labels peuvent relever d'une motivation sociétale pour l'amélioration de l'environnement ou la gestion durable des ressources, ou d'une qualité intrinsèque des produits issus de pratiques agroécologiques. On le voit, normes et labels pourront désormais englober des principes de natures très différentes.

La nécessité de caractériser et de gérer l'hétérogénéité dans toutes ses dimensions découle de ces constats : phénotypage numérique des individus (imageries, capteurs) venant compléter le génotypage déjà numérique (séquençage, bases de données), mais aussi caractérisation de l'environnement (données satellitaires, capteurs embarqués ou in situ), mise au point d'outils (logiciels, outils d'aide à la décision...) en appui à une conduite d'exploitation diversifiée. Des agroéquipements d'un nouveau genre peuvent y contribuer. Les capteurs et les outils de suivi des cultures ou des animaux sont encore peu utilisés pour aider au pilotage des systèmes, qu'ils soient ou non à visée agroécologique. Cette amélioration va au-delà de la simple acquisition des informations. Elle concerne la capacité à entretenir des processus en équilibre dynamique, notamment par la mesure de flux.

\section{De la collecte de données biologiques en masse à des dispositifs d'une nature nouvelle}

LA PRISE EN COMPTE D'INTERACTIONS À DIMENSIONS multiples induit un besoin massif en données nouvelles. Il faut mieux connaître les dynamiques des agroécosystèmes, changer d'échelle pour expérimenter comme pour observer, et surtout combiner les deux. Les apports de l'agriculture numérique, les méthodes de proxi-détection et de télédétection, l'analyse spatio-temporelle de données (écologie spatiale, statistiques spatio-temporelles, approches relevant du big data...) sont essentiels. Les données doivent plus encore s'appuyer sur des capteurs biologiques, des suivis à des fins de connaissance et de pilotage des systèmes, afin d'approcher la complexité des systèmes en agroécologie, riches par nature en interactions biotiques et abiotiques. Le renforcement de l'évaluation multicritère est utile pour pouvoir internaliser, à l'échelle de l'exploitation agricole, des externalités actuellement non prises en considération, comme la biodiversité.

Les dispositifs expérimentaux peuvent y contribuer en renforçant la collecte d'informations par l'instrumentation et l'acquisition de données numériques. Par exemple, on gagnerait à généraliser le génotypage des individus (animaux, plantes, arbres) avec un jeu de marqueurs communs et définis par espèce, pour intégrer la diversité génétique dans les protocoles visant à tester des modes de conduite, et ainsi quantifier la diversité présente et la comparer entre protocoles. La plupart des expérimentations systèmes ${ }^{64}$ mises en

64. Voir https://www6.inra.fr/experimentations-systeme/Definition-experimentation-systeme. 
avant pour la transition agroécologique de l'exploitation agricole n'intègrent pas encore la dimension génétique. De ce fait, le choix des génotypes est généralement occulté ou réalisé par défaut. Les dispositifs expérimentaux de la recherche pourraient également mieux s'attacher à caractériser les interactions biologiques, avec le développement par exemple d'approches fonctionnelles à même de déterminer les traits majeurs de la réponse des individus aux conditions de milieu (sols, climats, états hydriques, modes de conduite...). Les dispositifs expérimentaux ne suffiront pas, car ils n'offrent pas assez de combinaisons pour tester tous les niveaux d'interactions identifiés ! Même si des initiatives innovantes et très intégratrices existent, par exemple celle de créer des paysages agroécologiques expérimentaux, il subsistera toujours une variabilité, voire un écart, entre les pratiques mises en œuvre au sein de dispositifs expérimentaux et celles des conditions réelles des exploitations. De nouvelles stratégies doivent donc être imaginées, qui combinent les expérimentations menées par la recherche et la mobilisation d'autres sources de données, par exemple celles de dispositifs tels que le réseau des fermes Dephy, en les faisant éventuellement évoluer (nature des pratiques testées, nature des données collectées...). Davantage de recherches devront être développées sur un mode participatif, afin de valoriser la connaissance développée au sein de réseaux d'acteurs, d'initiatives pilotées par la société, sur un mode dit «actor-driven » et « citizen-driven ». Des réseaux de fermes et d'autres dispositifs coconstruits entre la recherche et les agriculteurs sont particulièrement utiles à l'étude de l'adaptation et de la transition des activités agricoles. Dans le cas de la sélection génétique évoqué ci-dessus, l'industrie de la sélection et les agriculteurs doivent être associés pour travailler sur un système changeant (environnement et espèces d'intérêt).

Des « dispositifs d'innovation ouverte », ou living labs, dans lesquels citoyens, habitants, usagers sont considérés comme des acteurs clés des processus de recherche et d'innovation, peuvent être adossés aux dispositifs de la recherche autour d'expérimentations de systèmes agroécologiques, ou mis en place au sein de projets d'innovation territoriale. Ils sont de plus en plus mis en avant et développés pour : analyser les dynamiques d'échanges de savoirs entre expérimentateurs, agriculteurs, acteurs des filières et des territoires, à partir d'une diversité d'expériences singulières et locales ; utiliser l'analyse multicritère pour mettre en évidence les points forts et faibles des différents systèmes testés, en fonction des critères analysés (agronomiques, zootechniques, environnementaux, économiques, sociaux), à différents pas de temps (début et fin de transition, système en rythme de croisière), dans différents contextes (prix des intrants et des produits, aléas économiques, climatiques...) ; coconstruire des indicateurs et des scénarios avec les partenaires ; produire des connaissances, au-delà des dimensions de production, sur ce qu'est l'agroécologie du point de vue de la gestion de l'incertitude, de l'anticipation, du rapport au milieu, à la santé végétale ou animale, etc.

Ces dispositifs, qu'ils soient expérimentaux, d'innovation ouverte ou hybrides, conduisent souvent à des données incomplètes ou obtenues sur des effectifs limités, ce qui nécessite l'adaptation des méthodes d'analyse des données. La réflexion doit se poursuivre 
pour mieux collecter et utiliser des données actuellement dispersées ou non compatibles entre elles. Cet enjeu, déjà identifié pour les dispositifs expérimentaux de la recherche, est plus critique encore pour les dispositifs impliquant des acteurs externes, le recueil des données étant pour eux l'objet de compromis, entre le temps de collecte et l'utilité des données dans le pilotage de leur activité.

Des expérimentations et des observations en sciences humaines et sociales, à grande échelle, relatives aux perceptions, aux freins et leviers, aux politiques publiques doivent se généraliser, être plus encore intégrées dès la coconception des actions. À titre d'exemple, les recherches sur les différentes fonctions assurées par le paysage demeurent compartimentées, les sciences économiques et sociologiques étant souvent absentes ou intégrées en aval des recherches.

\section{Appréhender le risque et l'incertitude : modélisation et partage d'expériences}

LA TRANSITION AGRoÉcologiQUe ENTRAîne une remise en cause de pratiques bien établies et contrôlées par l'apport d'intrants, sans que soit définis d'avance une cible, un système meilleur ou dominant. Ce changement représente une importante prise de risque, au niveau de chaque exploitant, et probablement plus encore au niveau d'une filière qui va devoir gérer une plus grande diversité d'acteurs, de produits et de risques. Les conséquences se situent aussi au niveau du consommateur, qui peut choisir d'encourager ou d'ignorer la diversification des systèmes selon son comportement d'acheteur.

Trois approches paraissent incontournables :

- la modélisation, pour augmenter la capacité prédictive de l'évolution des systèmes agricoles à différentes échelles de temps ; cette modélisation doit s'assortir d'approches sur l'incertitude pour mieux piloter des agrosystèmes nouveaux ; elle doit aider à identifier des impasses, des risques, des capacités de robustesse et de résilience ;

- l'apprentissage collectif, par le partage d'expériences, de savoirs d'origines diverses, s'inscrivant dans une démarche de sciences participatives, pour enrichir les degrés d'innovation et leur test dans différentes conditions ;

- l'analyse de la perception et l'objectivation du risque, de son accompagnement du point de vue socio-économique.

\section{Le nécessaire changement d'échelle et de niveau d'organisation}

L'AGRó́cologIE DEMANDE DE TENIR COMPTE D'OBJECTIFS de court terme (besoin immédiat de la plante en azote, régulation des mauvaises herbes qui concurrencent la culture...) et de moyen terme (maintien de la fertilité des sols, inoculum de ravageurs, banques de 
graines...). Du fait du principe de réduction des intrants, les solutions pour réduire fortement une population de bioagresseurs qui serait devenue trop abondante n'existent plus en agroécologie. Les déficits, les accumulations, toutes les dynamiques doivent donc mieux s'anticiper en prenant en compte les courts et moyens termes. D'autres services écosystémiques que la seule production de biomasse sont visés. La gestion des activités agricoles n'est donc plus "prédéterminée ", mais adaptative : les objectifs et règles de décision peuvent évoluer en fonction des informations disponibles, sur le court terme comme sur le long terme, au fur et à mesure des variations de l'état du « système » et en arbitrant entre services écosystémiques.

Les systèmes menés en agroécologie vont davantage dépendre d'effets de voisinage, d'éléments du paysage que dans le cas de systèmes qui s'appuient moins sur les processus écologiques. La gestion devra donc prendre en compte des étendues spatiales supérieures à celles de la parcelle ou de l'exploitation. Les exploitations engagées dans l'agroécologie vont disposer de ressources (herbe, composts...) dans certaines portions de territoires qui pourront être utilisées à d'autres endroits. Les échanges seront plus importants à considérer. Dans le cas des pathogènes, des nutriments ou des bioagresseurs, il s'agira notamment de raisonner à l'échelle de métapopulations ou de paysages. Enfin, les acteurs de la collecte (coopératives) et des industries de transformation sont importants dans la transition agroécologique, notamment sur les questions de leur coordination au sein d'un territoire.

Ces questions de changements d'échelle sont complexes du fait que les solutions sont très situées dans le temps et l'espace, donc très dépendantes des conditions locales, s'inscrivant nécessairement dans le cadre d'une gestion adaptative. La généricité dans la mise en œuvre de transitions agroécologiques ne va pas résider dans des solutions techniques, mais plutôt dans les cadres et les outils pour favoriser la capacité d'adaptation des acteurs. Il s'agit aussi de monter en compétence dans le domaine de l'économie circulaire, en prenant en compte : une circularité des flux de la matière ; des business models innovants pour la favoriser ; une adaptation aux conditions locales accompagnant ces circularités et préservant les milieux. Les approches en écologie industrielle peuvent y contribuer, de même que les approches les plus récentes en analyse du cycle de vie qui visent une régionalisation du calcul des impacts environnementaux des produits agricoles et alimentaires. Une réflexion sur l'agro-logistique, en particulier sur l'impact environnemental du transport des produits issus de l'agroécologie, est aussi à mener.

Des projets de territoires d'innovation ont été développés et la dynamique initiée peut être mise à profit pour construire des actions inter et transdisciplinaires dans un contexte favorable aux interactions avec les porteurs d'enjeux. Les projets « Dijon, territoire modèle du système alimentaire durable de 2030 ", "Acteurs économiques et citoyens construisent les territoires viticoles responsables et innovants de demain ", "Terres de Sources ", "Champs du possible, villes du futur » abordent de manière plus ou moins forte des problématiques d'agroécologie. De ces initiatives territoriales, il faudra capitaliser, déterminer ce qui est généricité et spécifique, passer de ces initiatives ancrées sur des conditions et des objectifs locaux à d'autres échelles. 


\section{Perppectives}

Quelques thèmes de recherche essentiels n'ont pas été abordés dans cet ouvrage, liés notamment aux conditions de changement d'échelle, de généralisation des systèmes conduits en agroécologie.

- Si les transitions agroécologiques se mettent en place en France, en Europe, dans le monde, quelles en seront les conséquences sur les rendements, la production mondiale, la disponibilité alimentaire, en quantité et en qualité, notamment dans le contexte du changement climatique ? Les systèmes en agroécologie seront plus résilients à la variabilité climatique. Si pour certains ils seront plus productifs, pourvus de ressources en eau suffisantes, pour beaucoup d'autres des questions subsistent sur leur viabilité aux échelles des grandes régions du monde.

- Le travail en agriculture sera modifié, allégé par les technologies dans certaines situations, mais sans doute plus consommateur en temps dans beaucoup d'autres. Quelles seront les conséquences économiques et sociales, y compris sur le bien-être des populations rurales? - Les transitions agroécologiques se traduiront-elles par des coûts de l'alimentation plus élevés ? Les consommateurs auront-ils tous les moyens d'accéder aux produits issus de l'agroécologie ? Comment favoriser l'accès à ces produits ? Les systèmes publics de restauration collective accompagneront sans doute localement les évolutions, mais la généralisation de l'usage de ces produits aux échelles nationale, européenne et mondiale devra faire l'objet de travaux pour analyser les trajectoires, du local au global, des systèmes agricoles, des productions agricoles, des régimes alimentaires, des conséquences économiques et sociales. - La transition agroécologique des exploitations, l'évolution des filières et les modifications quantitatives et qualitatives des productions agricoles vont-elles entraîner une modification de la localisation des productions et conduire à des réorganisations du tissu industriel agroalimentaire national ?

- L'agroécologie place l'écologie comme moteur fonctionnel et garant de la résilience et de la durabilité de la transformation des systèmes de productions agricoles et alimentaires. Quelles seront les interactions avec les autres transitions attendues et leurs conséquences (transition énergétique, neutralité carbone, contribution à l'atténuation du changement climatique, préservation des ressources en eau, etc.) ? Y aura-t-il dans certains territoires, dans certaines situations pédoclimatiques, des synergies ou au contraire des antagonismes?

- Beaucoup de trajectoires agroécologiques sont pensées localement au niveau du territoire, en réunissant les acteurs autour de la table, mais il faut aussi une réflexion globale afin d'anticiper et de penser les conséquences à l'échelle de la planète. Quelles terres dégradées pourrait-on reconquérir ? Quels espaces pourraient être multifonctionnels, multiproductifs (productions végétales et/ou animales, bois...) ? La transition agroécologique peut-elle amener une vision nouvelle des espaces productifs, des productions agricoles et agroalimentaires?

Quoi qu'il en soit, il ne fait aucun doute que la recherche abordera, avec l'agroécologie, des champs nouveaux et une façon nouvelle de penser ses activités. 


\section{Contributeurs}

Thierry Caquet, INRAE, directeur scientifique Environnement (coordination et chapitre 3) Benoît Dedieu, INRAE, chef du département Terra (comité de pilotage et chapitre 2)

Cécile Détang-Dessendre, INRAE, Économie, directrice scientifique adjointe Agriculture (comité de pilotage et chapitre 1)

Pierre Dupraz, INRAE, Économie, UMR Smart, Rennes (comité de pilotage et chapitre 2)

Philippe Faverdin, INRAE, Système d'élevage, UMR Pegase Rennes (chapitre 6)

Chantal Gascuel, INRAE, Sol-Hydrologie, directrice scientifique adjointe Environnement et UMR SAS Rennes (coordination et chapitre 5)

Laurent Hazard, INRAE, agronome, UMR AGIR Toulouse (chapitre 2)

Philippe Hinsinger, INRAE, chef du département AgroEnv (comité de pilotage)

Françoise Lescourret, INRAE, Écologie des agroécosystèmes, UR PSH Avignon (chapitre 5) Isabelle Litrico-Chiarelli, INRAE, Génétique végétale, UR P3F (chapitre 4)

Françoise Médale, INRAE, cheffe du département Physiologie animale et système d'élevage (comité de pilotage)

Hervé Monod, INRAE, chef de département NUMM (chapitre 5)

Sandrine Petit, INRAE, Écologie du paysage et santé des plantes, UMR Agroécologie Dijon (chapitre 3)

Xavier Reboud, INRAE, Écologie et santé des plantes, direction scientifique Agriculture (comité de pilotage et chapitre 6)

Lionel Roques, INRAE, Modélisation des agroécosystèmes, UR Biosp Avignon (chapitre 5) Jean-François Soussana, INRAE, vice-président International (comité de pilotage)

Alban Thomas, INRAE, chef du département Éco Socio (comité de pilotage)

Michèle Tixier-Boichard, INRAE, Génétique animale, UMR GABI Jouy-en-Josas (comité de pilotage et chapitre 4)

Hugo de Vries, INRAE, chargé de mission, DS Alimentation et Bioéconomie (chapitre 1) 
Nous remercions l'ensemble des personnes qui ont participé aux groupes de travail dont les animateurs sont co-auteurs des chapitres de cet ouvrage.

L'intégration de l'agroécologie dans les systèmes agrialimentaires. Joël Abecassis, Stéphane Bellon, Jean-Marc Blazy, Alain Boissy, Céline Bonnet, Zohra BouamraMechemache, Douadia Bougherara, Cécile Détang-Dessendre, Michel Duclos, Michel Duru, Alexandre Gohin, Bernard Kurek, Claire Lamine, Marianne Le Bail, Marie-Benoît Magrini, Ludovic Montastruc, Christine de Sainte Marie, Jean-Marc Meynard, Didier Raboisson, Hugo de Vries.

La transition agroécologique de l'exploitation agricole. Jacques-Éric Bergez, Alain Carpentier, Marianne Cerf, Benoît Dedieu, Pierre Dupraz, Jérôme Enjalbert, Anne Farruggia, Laurent Hazard, Claire Lamine, Amandine Lurette, Guillaume Martin, Roger Martin-Clouaire, Mireille Navarrete, Sandra Novak, Lorene Prost, Arnaud Reynaud.

Valoriser les processus écologiques, hydrobiogéochimiques dans des paysages multifonctionnels. Stéphanie Aviron, Thierry Caquet, Marc Deconchat, Sabrina Gaba, Chantal Gascuel, Hervé Jactel, Mourad Hannachi, Claire Lavigne, Vincent Martinet, Julien Papaïx, Sandrine Petit, Olivier Plantard, Lionel Ranjard, Marc Voltz.

Valoriser la diversité génétique en sélection végétale et animale. Jean-Pierre Bidanel, Nathalie Couix, Pascal Croiseau, Christian Ducrot, Pauline Ezzano, Hélène Freville, Arnaud Gauffreteau, Dominique Hazard, Pierre-Benoît Joly, Bernard Kurek, François Lefèvre, Stéphane Lemarié, Isabelle Litrico-Chiarelli, Valérie Mechin, Laurence Moreau, Jean-Benoît Morel, Laurence Puillet, Michèle Tixier-Boichard, Nicolas Verzelen.

Modéliser les interactions du vivant, en lien avec les milieux et les contextes socioéconomiques. David Bohan, Évelyne Costes, Pierre Courtois, Frédéric Fabre, Philippe Faverdin, Alain Franc, Chantal Gascuel, Thierry Hoch, Françoise Lescourret, Hervé Monod, Florence Phocas, Lionel Roques, Jean-Philippe Steyer, Marc Tchamitchian.

Quelle contribution des agroéquipements et du numérique à l'agroéocologie ? Frédéric Baret, Patrick Bertuzzi, André Chanzy, Philippe Faverdin, Nathalie Gandon, Frederick Garcia, Raphaël Guatteo, Alexandre Joannon, Morgan Meyer, Xavier Reboud, Christophe Staub, Pierre-Francois Vaquie. 

Édition : Juliette Blanchet

Infographie : Clémence Cautain

Mise en pages : Graph'M 
L'agroécologie a été choisie par l'Inra comme l'un des chantiers de prospective interdisciplinaire destinés à identifier les fronts de recherche en réponse à de grands défis sociétaux. Quatre-vingts chercheurs ont dressé un bilan, proposé des pistes de recherche pour l'agroécologie, et cet ouvrage en synthétise les principales conclusions.

L'agroécologie, en tant que discipline scientifique, remettant l'écologie au centre de la conception des systèmes agricoles, est maintenant bien présente. Diversifier le vivant dans des agroécosystèmes est une visée à large spectre pour les rendre plus robustes, plus résilients. Les recherches en génétique et en écologie du paysage sont mobilisées pour que l'agroécologie utilise des leviers de la parcelle au paysage. La modélisation des systèmes agroécologiques se développe pour mieux comprendre les interactions biotiques et abiotiques multiples, les prédire, et commencer à piloter certains systèmes. La diversification du vivant dans la production agricole (espèces, variétés, successions culturales, etc.) conduit à des produits plus variés. Les conséquences sont importantes sur les filières, ou plus exactement sur les systèmes agri-alimentaires, allant du mode de production aux produits consommés. Ces changements multi-échelles s'inscrivent sur le long terme. La transition agroécologique, adaptative, se coconstruisant avec les acteurs, est en soi un sujet de recherche, et pourra s'appuyer sur des dispositifs expérimentaux, des exploitations agricoles, des territoires d'innovation.

Thierry Caquet est écologue-écotoxicologue, directeur scientifique Environnement à INRAE.

Chantal Gascuel est hydropédologue, spécialiste des cycles biogéochimiques et des paysages, directrice scientifique adjointe à INRAE.

Michèle Tixier-Boichard est généticienne, spécialisée en génétique animale, ex-directrice scientifique adjointe à INRAE.

En couverture : plateforme d'expérimentation agroécologique à l'échelle du paysage (CA-SYS, INRAE), été 2019. (C) Brice Mosa, INRAE. 